\title{
FIELDS DEFINABLE IN THE FREE GROUP
}

\author{
AYALA DENTE BYRON AND RIZOS SKLINOS
}

Abstract. We prove that no infinite field is definable in the theory of the free group.

\section{INTRODUCTION}

After the work of Sela [18 and Kharlampovich-Myasnikov 6] leading to the positive answer to Tarski's question, there is an increasing model theoretic interest in the first-order theory of nonabelian free groups.

Although Sela proved that any definable set is equivalent to a boolean combination of $\forall \exists$-definable sets we are far from understanding these "basic" sets. According to Sela these sets admit a natural geometric interpretation, but admittedly neither geometers nor logicians have absorbed the sophisticated techniques that occur in his voluminous work. Thus, in principle, it is hard to determine whether a subset of some cartesian power of a nonabelian free group is definable or not.

Moreover, starting from Zilber's seminal work towards understanding uncountably categorical theories via some naturally defined pregeometries (see [23]), questions about what kind of groups are definable or whether an infinite field is definable in a given first order theory have become important within the community of model theorists.

Recently, some positive results along this line of thought have appeared. The following theorem has been proved independently in [7] and [9].

Theorem 1.1. The only definable proper subgroups of a torsion-free hyperbolic group are cyclic.

When it comes to infinite definable fields in some nonabelian free group, intuitively speaking, one expects to find none. To the best of our knowledge this was first posed as a conjecture in [13. This problem proved very hard to tackle, and the only positive result towards its solution had been the following theorem proved in the thesis [20] of the second-named author.

Theorem 1.2. Let $\mathbb{F}_{n}$ be the free group of rank $n$. Let $\phi$ be a formula over $\mathbb{F}_{n}$. Suppose $\phi\left(\mathbb{F}_{n}\right) \neq \phi\left(\mathbb{F}_{\omega}\right)$, where $\phi(\mathbb{F})$ denotes the solution set of $\phi$ in $\mathbb{F}$. Then $\phi$ cannot be given definably an abelian group structure.

On the other hand, in many model theoretic questions concerning existing "configurations" in a first order theory one does not need to understand the exact set of solutions of a formula but just its rough "shape". Indeed in this vein there has been progress. A definable set (or a parametric family) can be endowed with an envelope that contains the definable set, and moreover it carries a natural geometric

Received by the editors January 16, 2019, and, in revised form, May 3, 2019.

2010 Mathematics Subject Classification. Primary 03C45, 03C60, 20E05, 20F06, $20 \mathrm{E} 08$.

(C)2019 by the authors under Creative Commons Attribution-Noncommercial 3.0 License (CC BY NC 3.0) 
structure from which we can read properties that when they hold "generically" for the envelope, they also hold for the definable set. The method of envelopes has been proved very useful in proving (weak) elimination of imaginaries (see [14]) but also in proving that the first order theory of the free group does not have the finite cover property (see [21]). We will utilize envelopes once more in order to confirm the above conjecture.

The main theorem of this paper is:

Theorem 1. Let $\mathbb{F}$ be a nonabelian free group. Then no infinite field is definable in $\mathbb{F}$.

Our proof is based on the following theorem, which is a consequence of the quantifier elimination procedure. We record it next in the simplest possible form.

Theorem 1.3 (Sela). Let $\mathbb{F}:=\mathbb{F}(\bar{a})$. Let $\mathcal{T}(G, \mathbb{F})$ be a hyperbolic tower where $G:=\langle\bar{u}, \bar{x}, \bar{a} \mid \Sigma(\bar{u}, \bar{x}, \bar{a})\rangle$ and let $\phi(\bar{x}, \bar{a})$ be a first order formula over $\mathbb{F}$. Suppose there exists a test sequence, $\left(h_{n}\right)_{n<\omega}: G \rightarrow \mathbb{F}$ for $\mathcal{T}(G, \mathbb{F})$ such that $\mathbb{F}=\phi\left(h_{n}(\bar{x}), \bar{a}\right)$.

Then for any test sequence, $\left(h_{n}^{\prime}\right)_{n<\omega}: G \rightarrow \mathbb{F}$, for $\mathcal{T}(G, \mathbb{F})$ there is $n_{0}$ such that $\mathbb{F}=\phi\left(h_{n}^{\prime}(\bar{x}), \bar{a}\right)$ for all $n>n_{0}$.

The notions of a hyperbolic tower and of a test sequence over it will be defined in sections 4 and 6, respectively.

On our way to proving the main theorem, we prove various implicit function theorems. We recall that Merzlyakov's original theorem stated:

Theorem 1.4 (Merzlyakov). Let $\mathbb{F}:=\mathbb{F}(\bar{a})$. Let $\Sigma(\bar{x}, \bar{y}, \bar{a}) \subset_{\text {finite }}\langle\bar{x}, \bar{y}\rangle * \mathbb{F}$ be a finite set of words. Suppose $\mathbb{F} \models \forall \bar{x} \exists \bar{y}(\Sigma(\bar{x}, \bar{y}, \bar{a})=1)$. Then there exists a "formal solution" $\bar{w}(\bar{x}, \bar{a}) \subseteq\langle\bar{x}\rangle * \mathbb{F}$ such that $\Sigma(\bar{x}, \bar{w}(\bar{x}, \bar{a}), \bar{a})$ is trivial in $\langle\bar{x}\rangle * \mathbb{F}$.

We use the quantifier $\exists<\infty$ to denote a nonempty and finite set of solutions of a formula $\mathbb{F}=\exists^{<\infty} x \phi(x)$ if and only if $0<|\{a \in \mathbb{F}|\mathbb{F}|=\phi(a)\}|<\infty$.

We obtain the following "generalisation" to an arbitrary formula, but only after strengthening the hypothesis and weakening the conclusion of Merzlyakov's theorem as follows.

Theorem 2. Let $\mathbb{F}:=\mathbb{F}(\bar{a})$. Let $\mathbb{F}=\forall \bar{x} \exists<\infty \bar{y} \phi(\bar{x}, \bar{y}, \bar{a})$ and assume there exist a test sequence $\left(\bar{b}_{n}\right)_{n<\omega}$ and a sequence of tuples $\left(\bar{c}_{n}\right)_{n<\omega}$ such that $\mathbb{F}=\phi\left(\bar{b}_{n}, \bar{c}_{n}, \bar{a}\right)$. Then there exists a tuple of words $\bar{w}(\bar{x}, \bar{a})$ in $\langle\bar{x}\rangle * \mathbb{F}$ such that for any test sequence $\left(\bar{b}_{n}^{\prime}\right)_{n<\omega}$ in $\mathbb{F}$ we have that there exists $n_{0}$ (that depends on the test sequence) with $\mathbb{F}=\phi\left(\bar{b}_{n}^{\prime}, \bar{w}\left(\bar{b}_{n}^{\prime}, \bar{a}\right), \bar{a}\right)$ for all $n>n_{0}$.

We remark that our main theorem implies, together with the elimination of the "exists infinitely many" quantifier $\exists^{\infty}$, that no infinite field is definable in any model of this theory.

The proof splits into two parts. Roughly speaking for any definable set $X$ we prove that either $X$ is internal to a finite set of centralizers or it cannot be given definably an abelian group operation; i.e., there is no definable set $Y \subset X \times X \times X$ such that $Y$ is the graph of an abelian group operation on $X$. To conclude that no infinite field is definable we prove that centralizers of nontrivial elements are one-based.

The paper is structured as follows: in the next section we take the opportunity to recall some basic geometric stability theory and introduce the reader to the 
results that will allow us to conclude our theorem in the case a definable set is "coordinated" by a finite set of centralizers.

The following section contains introductory material that concerns Bass-Serre theory as well as results for a special class of groups called limit groups. The material here is by no means original and is certainly well known. Since in many of our arguments we will use actions on trees or normal forms for groups that admit a graph of groups splitting, we hope that this section will provide an adequate background for the uninitiated.

Section 4 contains many of the core notions which are important in this paper. We start by explaining when a group admits the structure of a tower and then we continue by introducing a construction that leads to the notion of a twin tower. Twin towers will play a fundamental role in our main proof.

In sections 5 and [6] we record and extend some constructions and results of Sela that appear in [16, [17, and [14]. Here the reader will find all the technical apparatus that makes our main proof possible. Theorems 6.25, 6.32, and 6.34 lie in the core of our result.

Finally, in the last section we bring everything together and we prove the main result. We split the proof into two cases: the abelian case and the nonabelian case. The abelian case is resolved using geometric stability, while the nonabelian case is resolved using geometric group theory. We have also added an example, which we call the hyperbolic case, where our proof is free of certain technical phenomena, so the reader could clearly see the idea behind it.

\section{Some Geometric Stability}

In this section we provide some quick model theoretic background on stable theories. A gentle introduction to stability and forking independence has been given in [10], so to avoid repetition we refer the reader there. Our main focus in this paper will be on geometric stability and in particular on the notion of onebasedness. For more details the reader can consult [11. We work in the monster model $\mathbb{M}$ of a stable theory $T$.

Definition 2.1. A definable set $X$ (in $\mathbb{M}$ ) is called weakly normal if for every $a \in X$ only finitely many translates of $X$ under Aut(M) contain $a$.

Definition 2.2. The first order theory $T$ is one-based if every definable set (in $\mathbb{M}$ ) is a boolean combination of weakly normal definable sets.

The simplest example of a one-based theory is the theory of a vector space $\left(V,+, 0,\left\{r_{k}\right\}_{k \in K}\right)$ over a field $K$, where for each $k \in K, r_{k}$ is a function symbol which is interpreted in the structure as scalar multiplication by the element $k \in K$. In the same vein we have:

Fact 2.3. The theory of any abelian group (in the group language) is one-based.

For the purposes of our paper one-based theories have an important property proved by Pillay in [12.

Fact 2.4 (Pillay). Let $T$ be one-based. Then no infinite field is interpretable in $T$.

A set is interpretable if it is definable up to a definable equivalence relation. Thus, in particular no infinite field is definable in a one-based theory. 
For any definable set $X$ in $\mathbb{M}$ we can define the induced structure on $X, X^{\text {ind }}$, in the following way: the universe of the structure will be $X$, and for every definable set $Y$ in $\mathbb{M}$ we add a predicate $P_{Y}$ that corresponds to the intersection $Y \cap X$.

Definition 2.5. Let $X$ be a definable set in $\mathbb{M}$. Then $X$ is one-based if the first order theory of $X^{\text {ind }}$ is one-based.

We say that a family of definable sets $\mathcal{P}$ is $\emptyset$-invariant if the image of any definable set in $\mathcal{P}$ by an automorphism in $\operatorname{Aut}(\mathbb{M})$ is still in $\mathcal{P}$. Moreover, if $B$ is a small subset of $\mathbb{M}$, we say that $\bar{c} \models \mathcal{P} \uparrow B$ if $t p(\bar{c} / B)$ contains some definable set that belongs to $\mathcal{P}$. Finally, by $\bar{c} \underset{A}{\downarrow} B$, we mean that $\bar{c}$ is independent from $B$ over $A$ in the sense of forking independence.

Definition 2.6. A definable set $X$ (over some small subset $A \subset \mathbb{M}$ ) is $\mathcal{P}$-internal for some $\emptyset$-invariant family of definable sets $\mathcal{P}$ if for any $\bar{c} \in X$ there exists $B \supseteq A$ with $\bar{c} \underset{A}{\downarrow} B$ and $\bar{b}_{1}, \ldots, \bar{b}_{k}$ with $\bar{b}_{i} \models \mathcal{P} \uparrow B$ such that $\bar{c} \in \operatorname{dcl}\left(B, \bar{b}_{1}, \ldots, \bar{b}_{k}\right)$.

The following theorem has been proved by F. Wagner in [22].

Theorem 2.7 (Wagner). Let $X$ be a definable set and let $\mathcal{P}$ be a $\emptyset$-invariant family of one-based sets. If $X$ is $\mathcal{P}$-internal, then $X$ is one-based.

\section{Actions on trees}

The goal of this section is to present a structure theorem for groups acting on trees. In the first subsection we will be interested in group actions on simplicial trees. These actions can be analysed using Bass-Serre theory, and we will explain the duality between the notion of a graph of groups and these actions.

In the second subsection we will record the notion of a real tree and quickly describe some natural group actions on real trees.

3.1. Bass-Serre theory. Bass-Serre theory gives a structure theorem for groups acting on (simplicial) trees, i.e., acyclic connected graphs. It describes a group (that acts on a tree) as a series of amalgamated free products and HNN extensions. The mathematical notion that contains these instructions is called a graph of groups. For a complete treatment we refer the reader to [19].

We start with the definition of a graph.

Definition 3.1. A graph $G(V, E)$ is a collection of data that consists of two sets $V$ (the set of vertices) and $E$ (the set of edges) together with three maps:

- an involution ${ }^{-}: E \rightarrow E$, where $\bar{e}$ is called the inverse of $e$;

- $\alpha: E \rightarrow V$, where $\alpha(e)$ is called the initial vertex of $e$; and

- $\tau: E \rightarrow V$, where $\tau(e)$ is called the terminal vertex of $e$,

so that $\bar{e} \neq e$ and $\alpha(e)=\tau(\bar{e})$ for every $e \in E$.

Definition 3.2 (Graph of groups). A graph of groups $\mathcal{G}:=\left(G(V, E),\left\{G_{u}\right\}_{u \in V}\right.$, $\left.\left\{G_{e}\right\}_{e \in E},\left\{f_{e}\right\}_{e \in E}\right)$ consists of the following data:

- a graph $G(V, E)$;

- a family of groups $\left\{G_{u}\right\}_{u \in V}$; i.e., a group is attached to each vertex of the graph;

- a family of groups $\left\{G_{e}\right\}_{e \in E}$; i.e., a group is attached to each edge of the graph. Moreover, $G_{e}=G_{\bar{e}}$; 
- a collection of injective morphisms $\left\{f_{e}: G_{e} \rightarrow G_{\tau(e)} \mid e \in E\right\}$; i.e., each edge group comes equipped with two embeddings to the incident vertex groups.

The fundamental group of a graph of groups is defined as follows.

Definition 3.3. Let $\mathcal{G}:=\left(G(V, E),\left\{G_{u}\right\}_{u \in V},\left\{G_{e}\right\}_{e \in E},\left\{f_{e}\right\}_{e \in E}\right)$ be a graph of groups. Let $T$ be a maximal subtree of $G(V, E)$. Then the fundamental group, $\pi_{1}(\mathcal{G}, T)$, of $\mathcal{G}$ with respect to $T$ is the group given by the following presentation:

$$
\begin{array}{r}
\left\langle\left\{G_{u}\right\}_{u \in V},\left\{t_{e}\right\}_{e \in E}\right| t_{e}^{-1}=t_{\bar{e}} \quad \text { for } e \in E, t_{e}=1 \quad \text { for } e \in T, f_{e}(a)=t_{e} f_{\bar{e}}(a) t_{\bar{e}} \\
\text { for } \left.e \in E a \in G_{e}\right\rangle .
\end{array}
$$

Remark 3.4. It is not hard to see that the fundamental group of a graph of groups does not depend on the choice of the maximal subtree up to isomorphism (see [19, Proposition 20, p. 44]).

In order to give the main theorem of Bass-Serre theory we need the following definition.

Definition 3.5. Let $G$ be a group acting on a simplicial tree $T$ without inversions, denote by $\Lambda$ the corresponding quotient graph, and denote by $p$ the quotient map $T \rightarrow \Lambda$. A Bass-Serre presentation for the action of $G$ on $T$ is a triple $\left(T^{1}, T^{0},\left\{\gamma_{e}\right\}_{e \in E\left(T^{1}\right) \backslash E\left(T^{0}\right)}\right)$ consisting of

- a subtree $T^{1}$ of $T$ which contains exactly one edge of $p^{-1}(e)$ for each edge $e$ of $\Lambda$;

- a subtree $T^{0}$ of $T^{1}$ which is mapped injectively by $p$ onto a maximal subtree of $\Lambda$;

- a collection of elements of $G,\left\{\gamma_{e}\right\}_{e \in E\left(T^{1}\right) \backslash E\left(T^{0}\right)}$, such that if $e=(u, v)$ with $v \in T^{1} \backslash T^{0}$, then $\gamma_{e} \cdot v$ belongs to $T^{0}$.

Theorem 3.6. Suppose $G$ acts on a simplicial tree $T$ without inversions. Let $\left(T^{1}, T^{0},\left\{\gamma_{e}\right\}\right)$ be a Bass-Serre presentation for the action. Let $\mathcal{G}:=(G(V, E)$, $\left.\left\{G_{u}\right\}_{u \in V},\left\{G_{e}\right\}_{e \in E},\left\{f_{e}\right\}_{e \in E}\right)$ be the following graph of groups:

- $G(V, E)$ is the quotient graph given by $p: T \rightarrow \Gamma$;

- if $u$ is a vertex in $T^{0}$, then $G_{p(u)}=\operatorname{Stab}_{G}(u)$;

- if $e$ is an edge in $T^{1}$, then $G_{p(e)}=\operatorname{Stab}_{G}(e)$;

- if $e$ is an edge in $T^{1}$, then $f_{p(e)}: G_{p(e)} \rightarrow G_{\tau(p(e))}$ is given by the identity if $e \in T^{0}$ and by conjugation by $\gamma_{e}$ if not.

Then $G$ is isomorphic to $\pi_{1}(\mathcal{G})$.

Remark 3.7. The other direction of the above theorem also holds. Whenever a group $G$ is isomorphic to the fundamental group of a graph of groups then there is a natural way to obtain a simplicial tree $T$ and an action of $G$ on $T$ (see [19, section 5.3, p. 50]).

Among splittings of groups we will distinguish those with some special type vertex groups called surface type vertex groups. 
Definition 3.8. Let $G$ be a group acting on a tree $T$ without inversions and let $\left(T_{1}, T_{0},\left\{\gamma_{e}\right\}\right)$ be a Bass-Serre presentation for this action. Then a vertex $v \in T^{0}$ is called a surface type vertex if the following conditions hold:

- $\operatorname{Stab}_{G}(v)=\pi_{1}(\Sigma)$ for a connected compact surface $\Sigma$ with nonempty boundary, such that either the Euler characteristic of $\Sigma$ is at most -2 or $\Sigma$ is a once punctured torus.

- For every edge $e \in T_{1}$ adjacent to $v, \operatorname{Stab}_{G}(e)$ embeds onto a maximal boundary subgroup of $\pi_{1}(\Sigma)$, and this induces a one-to-one correspondence between the set of edges (in $T^{1}$ ) adjacent to $v$ and the set of boundary components of $\Sigma$.

We next follow 2 and define the notion of a generalized abelian decomposition (GAD).

Definition 3.9. A GAD of a group $G$ is a graph of groups $\mathcal{G}(V, E)$ with abelian edge groups such that $V$ is partitioned as $V_{S} \cup V_{A} \cup V_{R}$ where:

- each vertex in $V_{S}$ is a vertex of surface type for the corresponding action on a tree;

- each vertex group for a vertex in $V_{A}$ is noncyclic abelian; and

- each vertex group for a vertex $V_{R}$ is called rigid.

Definition 3.10 (Peripheral subgroup). Let $A$ be a vertex group of a GAD of $G,\left(\mathcal{G}(V, E),\left(V_{S}, V_{A}, V_{R}\right)\right)$, whose vertex is in $V_{A}$. Then we denote by $P(A)$ the subgroup of $A$ generated by all incident edge groups. Moreover the subgroup of $A$ that dies under every morphism $h: A \rightarrow \mathbb{Z}$ that kills $P(A)$ is called the peripheral subgroup and is denoted by $\bar{P}(A)$.

Definition 3.11 (Dehn twists). Let $H$ be a subgroup of $G$. Suppose $G$ splits as an:

- amalgamated free product $A *_{C} B$ so that $H$ is a subgroup of $A$. Let $g$ be an element in the centralizer of $C$ in $G$. Then a Dehn twist in $g$ is the automorphism fixing $A$ pointwise and sending each element $b$ of $B$ to $g b g^{-1}$.

- HNN-extension $A *_{C}$ so that $H$ is a subgroup of $A$. Let $g$ be an element in the centralizer of $C$ in $G$. Then a Dehn twist in $g$ is the automorphism fixing $A$ and sending the Bass-Serre element $t$ to $t g$.

Definition 3.12 (Relative modular automorphisms). Let $H$ be a subgroup of $G$. Let $\Delta:=\left(\mathcal{G}(V, E),\left(V_{S}, V_{A}, V_{R}\right)\right)$ be a GAD of $G$ in which $H$ can be conjugated into a vertex group. Then $\operatorname{Mod}_{H}(\Delta)$ is the subgroup of $\operatorname{Aut}_{H}(G)$ generated by:

- inner automorphisms;

- unimodular automorphisms of $G_{u}$ for $u \in V_{A}$ that fix the peripheral subgroup of $G_{u}$ and every other vertex group;

- automorphisms of $G_{u}$ for $u \in V_{S}$ coming from homeomorphisms of the corresponding surface that fix all boundary components;

- Dehn twists in elements of centralizers of edge groups, after collapsing the GAD to a one edge splitting in which $H$ is a subgroup of a vertex group.

Moreover we define the modular group of $G$ relative to $H, \operatorname{Mod}_{H}(G)$, to be the group generated by $\operatorname{Mod}_{H}(\Delta)$ for every GAD $\Delta$ of $G$. 
3.2. Actions on real trees. Real trees (or $\mathbb{R}$-trees) generalize simplicial trees in the following way.

Definition 3.13. A real tree is a geodesic metric space in which for any two points there is a unique arc that connects them.

When we say that a group $G$ acts on a real tree $T$ we will always mean an action by isometries.

Moreover, an action $G \curvearrowright T$ of a group $G$ on a real tree $T$ is called nontrivial if there is no globally fixed point and minimal if there is no proper $G$-invariant subtree. Lastly, an action is called free if for any $x \in T$ and any nontrivial $g \in G$ we have that $g \cdot x \neq x$.

We next collect some families of group actions on real trees.

Definition 3.14. Let $G \curvearrowright^{\lambda} T$ be a minimal action of a finitely generated group $G$ on a real tree $T$. Then we say:

(i) $\lambda$ is of discrete (or simplicial) type if every orbit G.x is discrete in T. In this case $T$ is simplicial, and the action can be analyzed using Bass-Serre theory.

(ii) $\lambda$ is of axial (or toral) type if $T$ is isometric to the real line $\mathbb{R}$ and $G$ acts with dense orbits; i.e., $\overline{G . x}=T$, for every $x \in T$.

(iii) $\lambda$ is of surface (or IET) type if $G=\pi_{1}(\Sigma)$ where $\Sigma$ is a surface with (possibly empty) boundary carrying an arational measured foliation and $T$ is dual to $\tilde{\Sigma}$; i.e., $T$ is the lifted leaf space in $\tilde{\Sigma}$ after identifying leaves of distance 0 (with respect to the pseudometric induced by the measure).

We will use the notion of a graph of actions in order to glue real trees equivariantly. We follow the exposition in [3, section 1.3].

Definition 3.15 (Graph of actions). A graph of actions $\left(G \curvearrowright T,\left\{Y_{u}\right\}_{u \in V(T)}\right.$, $\left.\left\{p_{e}\right\}_{e \in E(T)}\right)$ consists of the following data:

- a simplicial type action $G \curvearrowright T$;

- for each vertex $u$ in $T$ a real tree $Y_{u}$;

- for each edge $e$ in $T$, an attaching point $p_{e}$ in $Y_{\tau(e)}$.

Moreover:

(1) $G$ acts on $R:=\left\{\coprod Y_{u}: u \in V(T)\right\}$ so that $q: R \rightarrow V(T)$ with $q\left(Y_{u}\right)=u$ is $G$-equivariant;

(2) for every $g \in G$ and $e \in E(T), p_{g \cdot e}=g \cdot p_{e}$.

To a graph of actions $\mathcal{A}:=\left(G \curvearrowright T,\left\{Y_{u}\right\}_{u \in V(T)},\left\{p_{e}\right\}_{e \in E(T)}\right)$ we can assign an $\mathbb{R}$ tree $Y_{\mathcal{A}}$ endowed with a $G$-action. Roughly speaking this tree will be $\coprod_{u \in V(T)} Y_{u} / \sim$, where the equivalence relation $\sim$ identifies $p_{e}$ with $p_{\bar{e}}$ for every $e \in E(T)$. We say that a real $G$-tree $Y$ decomposes as a graph of actions $\mathcal{A}$ if there is an equivariant isometry between $Y$ and $Y_{\mathcal{A}}$.

Interesting actions on real trees can be obtained by sequences of morphisms from a finitely generated group to a free group. We explain how in the next subsection.

3.3. The Bestvina-Paulin method. The construction we are going to record is credited to Bestvina [1] and Paulin [8] independently.

We fix a finitely generated group $G$ and we consider the set of nontrivial equivariant pseudometrics $d: G \times G \rightarrow \mathbb{R}^{\geq 0}$, denoted by $\mathcal{E D}(G)$. We equip $\mathcal{E D}(G)$ with 
the compact-open topology (where $G$ is given the discrete topology). Note that convergence in this topology is given by

$$
\left.\left(d_{i}\right)_{i<\omega} \rightarrow d \quad \text { if and only if } d_{i}(1, g) \rightarrow d(1, g) \quad \text { (in } \mathbb{R}\right) \text { for any } g \in G \text {. }
$$

It is not hard to see that $\mathbb{R}^{+}$acts cocompactly on $\mathcal{E D}(G)$ by rescaling; thus the space of projectivised equivariant pseudometrics on $G$ is compact.

We also note that any based $G$-space $(X, *)$ (i.e., a metric space with a distinguished point equipped with an action of $G$ by isometries) gives rise to an equivariant pseudometric on $G$ as follows: $d(g, h)=d_{X}(g \cdot *, h \cdot *)$.

We say that a sequence of $G$-spaces $\left(X_{i}, *_{i}\right)_{i<\omega}$ converges to a $G$-space $(X, *)$ if the corresponding pseudometrics induced by $\left(X_{i}, *_{i}\right)$ converge to the pseudometric induced by $(X, *)$ in $\mathcal{P} \mathcal{E} \mathcal{D}(G)$.

A morphism $h: G \rightarrow H$ where $H$ is a finitely generated group induces an action of $G$ on $\mathcal{X}_{H}$ (the Cayley graph of $H$ ) in the obvious way, thus making $\mathcal{X}_{H}$ a $G$-space. We have:

Lemma 3.16. Let $\mathbb{F}$ be a nonabelian free group. Let $\left(h_{n}\right)_{n<\omega}: G \rightarrow \mathbb{F}$ be a sequence of pairwise nonconjugate morphisms. Then for each $n<\omega$ there exists a base point $*_{n}$ in $\mathcal{X}_{\mathbb{F}}$ such that the sequence of $G$-spaces $\left(\mathcal{X}_{\mathbb{F}}, *_{n}\right)_{n<\omega}$ has a convergent subsequence to a real $G$-tree $(T, *)$, where the action of $G$ on $T$ is nontrivial.

\subsection{Limit groups.}

Definition 3.17. Let $G$ be a group and let $\left(h_{n}\right)_{n<\omega}: G \rightarrow \mathbb{F}$ be a sequence of morphisms. Then the sequence $\left(h_{n}\right)_{n<\omega}$ is called convergent if for every $g \in G$, there exists $n_{g}$ such that either $h_{n}(g) \neq 1$ for all $n>n_{g}$ or $h_{n}(g)=1$ for all $n>n_{g}$.

Moreover, if $\left(h_{n}\right)_{n<\omega}: G \rightarrow \mathbb{F}$ is a convergent sequence, then we define its stable kernel $\operatorname{Ker}_{n}:=\left\{g \in G \mid g\right.$ is eventually killed by $\left.h_{n}\right\}$.

Definition 3.18. Let $G$ be a finitely generated group. Then $G$ is a limit group if there exists a convergent sequence $\left(h_{n}\right)_{n<\omega}: G \rightarrow \mathbb{F}$ with trivial stable kernel.

Limit groups can be given a constructive definition. To this end we define:

Definition 3.19. Let $\Delta:=\left(\mathcal{G}(V, E),\left(V_{S}, V_{A}, V_{R}\right)\right)$ be a GAD of a group $G$. For each $G_{v}$ with $v \in V_{R}$ we define its envelope $\tilde{G}_{v}$ in $\Delta$ in the following way: for every $a \in V_{A}$ we replace $G_{a}$ in $\mathcal{G}(V, E)$ by its peripheral subgroup. Then $\tilde{G}_{v}$ is the group generated by $G_{v}$ together with the centralizers of incident edge groups.

Definition 3.20 (Strict morphisms). Let $\eta: G \rightarrow L$ be an epimorphism and let $\Delta:=\left(\mathcal{G}(V, E),\left(V_{S}, V_{A}, V_{R}\right)\right)$ be a GAD of $G$ in which every edge group is maximal abelian in at least one vertex group of the one edged splitting induced by the edge. Then $\eta$ is strict with respect to $\Delta$ if the following hold:

- $\eta$ is injective on each edge group;

- $\eta$ is injective on $\tilde{G}_{v}$ for every $v \in V_{R}$;

- $\eta$ is injective on the peripheral subgroup of each abelian vertex group;

- $\eta\left(G_{s}\right)$ is not abelian for every $s \in V_{S}$.

Definition 3.21. A group $L$ is a constructive limit group if it belongs to the following hierarchy of groups defined recursively.

Base step. Level 0 consists of finitely generated free groups. 
Recursive step. A group $G$ belongs to level $i+1$ if it is either the free product of two groups that belong to level $i$ or there exists a GAD, $\Delta$, for $G$ and a strict map $\eta: G \rightarrow H$ with respect to $\Delta$ onto some $H$ that belongs to level $i$.

The following theorem appears as Theorem 5.12 in [15].

Theorem 3.22 (Sela). Let $L$ be a finitely generated group. Then $L$ is a limit group if and only if it is a constructive limit group.

3.5. Graded limit groups. A graded limit group is a limit group together with a distinguished finitely generated subgroup. We will be interested in a special kind of graded limit group called solid limit groups. Solid and rigid limit groups were defined in [15, Definition 10.2], and the corresponding notion in the work of Kharlampovich-Myasnikov [6] is the notion of a group without sufficient splittings.

Definition 3.23. Let $G$ be a limit group which is freely indecomposable with respect to a finitely generated subgroup $H$. We fix a finite generating set $\Sigma$ for $G$ and a basis $\bar{a}$ for $\mathbb{F}:=\mathbb{F}(\bar{a})$. A morphism $h: G \rightarrow \mathbb{F}$ is short with respect to $\operatorname{Mod}_{H}(G)$ if for every $\sigma \in \operatorname{Mod}_{H}(G)$ and every $g \in \mathbb{F}$ that commutes with $h(H)$ we have that $\max _{s \in \Sigma}|h(s)|_{\mathbb{F}} \leq \max _{s \in \Sigma}|\operatorname{Conj}(g) \circ h \circ \sigma|_{\mathbb{F}}$.

Definition 3.24. Let $G$ be a limit group which is freely indecomposable with respect to a finitely generated subgroup $H$. Let $\left(h_{n}\right)_{n<\omega}: G \rightarrow \mathbb{F}$ be a convergent sequence of short morphisms with respect to $\operatorname{Mod}_{H}(G)$. Then we call $G / \operatorname{Ker} h_{n}$ a shortening quotient of $G$ with respect to $H$.

Definition 3.25 (Solid limit group). Let $S$ be a limit group and let $H$ be a finitely generated subgroup of $S$. Suppose $S$ is freely indecomposable with respect to $H$. Then $S$ is solid with respect to $H$ if there exists a shortening quotient of $S$ with respect to $H$ which is isomorphic to $S$.

Example 3.26. The surface group $\left\langle x_{1}, x_{2}, \ldots, x_{2 k}, e_{1}, e_{2}, \ldots, e_{2 m}\right|\left[x_{1}, x_{2}\right]\left[x_{3}, x_{4}\right]$ $\left.\ldots\left[x_{2 k-1}, x_{2 k}\right]\left[e_{1}, e_{2}\right] \cdots\left[e_{2 m-1}, e_{2 m}\right]\right\rangle$ is a solid limit group with respect to the subgroup $\left\langle e_{1}, \ldots, e_{2 m}\right\rangle$.

Definition 3.27. Let $S l d$ be a solid limit group with respect to a finitely generated subgroup $H$ with generating set $\Sigma_{H}$. Let $\left(h_{n}\right)_{n<\omega}:$ Sld $\rightarrow \mathbb{F}$. We call $\left(h_{n}\right)_{n<\omega}$ a flexible sequence if for every $n$ either:

- the morphism $h_{n}=h_{n}^{\prime} \circ \eta_{n}$, where $\eta_{n}: S l d \rightarrow \mathbb{F} * \Gamma$ for some group $\Gamma, H$ is mapped onto $\mathbb{F}$ by $\eta_{n}, h_{n}^{\prime}: \mathbb{F} * \Gamma \rightarrow \mathbb{F}$ stays the identity on $\mathbb{F}$, and $\eta_{n}$ is short with respect to $\operatorname{Mod}_{H}(S l d)$ or

- the morphism $h_{n}$ is short with respect to $\operatorname{Mod}_{H}(S l d)$ and moreover

$$
\max _{g \in B_{n}}\left|h_{n}(g)\right|_{\mathbb{F}}>2^{n}\left(1+\max _{s \in \Sigma_{H}}\left|h_{n}(s)\right|_{\mathbb{F}}\right),
$$

where $B_{n}$ is the ball of radius $n$ in the Cayley graph of Sld.

If $\left(h_{n}\right)_{n<\omega}: S l d \rightarrow \mathbb{F}$ is a convergent flexible sequence, then we call $\operatorname{Sld} / \operatorname{Ker} h_{n}$ a flexible quotient of $S l d$.

It is not hard to see, using the shortening argument, that flexible quotients are proper. Moreover one can define a partial order and an equivalence relation on the class of flexible quotients of a solid limit group. Let $S l d$ be a solid limit group with respect to a finitely generated subgroup $H$ and let $\eta_{i}: S l d \rightarrow Q_{i}$ for $i \leq 2$ be flexible quotients with their canonical quotient maps. Then $Q_{2} \leq Q_{1}$ if ker $\eta_{1} \subseteq \operatorname{ker} \eta_{2}$, and $Q_{1} \sim Q_{2}$ if there exists $\sigma \in \operatorname{Mod}_{H}(S l d)$ such that $\operatorname{ker}\left(\eta_{1} \circ \sigma\right)=\operatorname{ker} \eta_{2}$. 
For the following theorem see the discussion before Definition 10.5 in [15].

Theorem 3.28 (Sela). Let Sld be a solid limit group with respect to a finitely generated subgroup $H$. Assume that Sld admits a flexible quotient. Then there exist finitely many classes of maximal flexible quotients.

A morphism from a solid limit group to a free group that does not factor through one of the maximal flexible quotients (after precomposition by a modular automorphism) is called a solid morphism; otherwise it is called flexible (see [15, Definition 10.6]).

\section{TOWERS}

In this section we are interested in limit groups that have a very special structure, namely, the structure of a tower. A tower is built recursively adding floors to a given basis, which is taken to be a free product of fundamental groups of surfaces with free abelian groups. Each floor is built by "gluing" a finite set of surface flats and abelian flats to the previous one following specific rules, to be made precise in the next subsection. The corresponding notion in the work of Kharlampovich-Myasnikov is the notion of an NTQ group, i.e., the coordinate group of a nondegenerate triangular quasiquadratic system of equations (see [4, Definition 9]).

Limit groups that admit the structure of a tower play a significant role in the proof of the elementary equivalence of nonabelian free groups. This class of limit groups is connected with generalizations of Merzlyakov's theorem as proved in [16] and [5]. We will analyse and further expand this connection in section 6 .

4.1. The construction of a tower. We start with defining the notion of a surface flat.

Definition 4.1 (Surface flat). Let $G$ be a group and let $H$ be a subgroup of $G$ (see Figure 10. Then $G$ has the structure of a surface flat over $H$ if $G$ acts minimally on a tree $T$ and the action admits a Bass-Serre presentation $\left(T^{1}, T^{0},\left\{\gamma_{e}\right\}\right)$ such that:

- the set of vertices of $T^{1}$ is partitioned into two sets, $\{v\}$ and $V$, where $v$ is a surface type vertex;

- $T^{1}$ is bipartite between $v$ and $V\left(T^{1}\right) \backslash\{v\}$;

- $H$ is the free product of the stabilizers of vertices in $V$;

- either there exists a retraction $r: G \rightarrow H$ that sends $\operatorname{Stab}_{G}(v)$ to a nonabelian image or $H$ is cyclic and there exists a retraction $r^{\prime}: G * \mathbb{Z} \rightarrow H * \mathbb{Z}$ which sends $\operatorname{Stab}_{G}(v)$ to a nonabelian image.

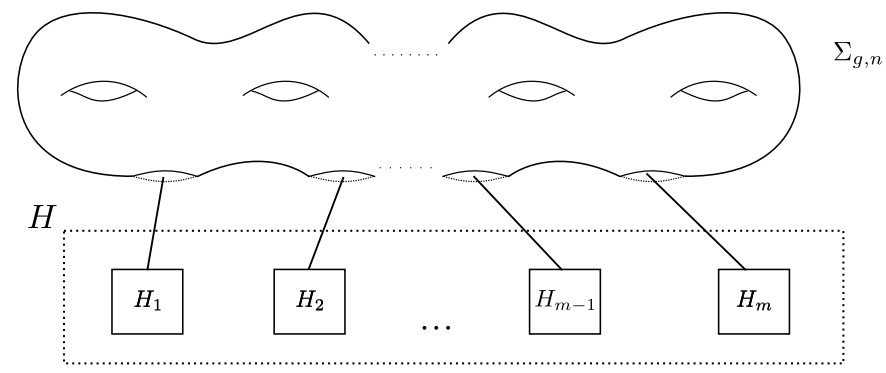

Figure 1. A surface flat. 
Remark 4.2. A more concise way to refer to a group $G$ that has the structure of a surface flat over a subgroup $H$ is to say that $G$ is obtained from $H$ by gluing a surface $\Sigma_{g, n}$ along its boundary onto the subgroups $\left\{E_{i}: i \leq n\right\}$ of $H$.

Example 4.3. The surface group $\pi_{1}\left(\Sigma_{g}\right)=\left\langle x_{1}, \ldots, x_{2 g} \mid\left[x_{1}, x_{2}\right] \cdot \ldots \cdot\left[x_{2 g-1}, x_{2 g}\right]\right\rangle$ has the structure of a surface flat over $\mathbb{F}_{g}:=\left\langle x_{1}, \ldots, x_{g}\right\rangle$ (see Figure 21).

Concisely, $\pi_{1}\left(\Sigma_{g}\right)$ is obtained from $\mathbb{F}_{g}$ by gluing $\left\langle b, x_{g+1}, \ldots, x_{2 g}\right| b^{-1} \cdot\left[x_{g+1}, x_{g+2}\right]$ $\left.\cdot \ldots \cdot\left[x_{2 g-1}, x_{2 g}\right]\right\rangle$ along its boundary onto $\left\langle\left[x_{1}, x_{2}\right] \ldots\left[x_{g-1}, x_{g}\right]\right\rangle$.

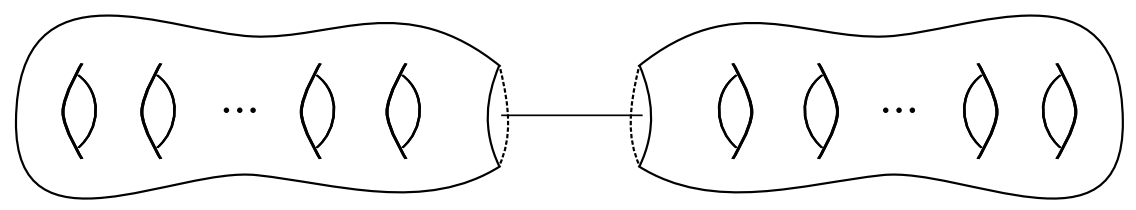

Figure 2. An example of a surface group that has the structure of a surface flat over a free group.

We similarly define the notion of an abelian flat.

Definition 4.4. Let $G$ be a group and let $H$ be a subgroup of $G$. Then $G$ has the structure of an abelian flat over $H$ if $G$ acts minimally on a tree $T$ with a single orbit of edges, and the action admits a Bass-Serre presentation $\left(T^{1}=T^{0}, T^{0}\right)$ so that if $e=\{u, v\}$ is the unique edge in $T^{0}$, then $\operatorname{Stab}_{G}(u)=H, \operatorname{Stab}_{G}(e)$ is a maximal abelian subgroup of $H$, which we call the peg of the abelian flat, and $\operatorname{Stab}_{G}(v)=\operatorname{Stab}_{G}(e) \oplus \mathbb{Z}^{m}$ for some $m<\omega$ (see Figure 3).

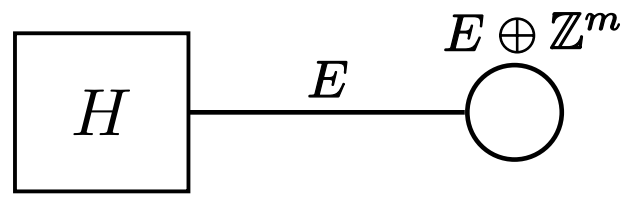

Figure 3. An abelian flat.

Remark 4.5. A more concise way to refer to a group $G$ that has the structure of an abelian flat over a subgroup $H$ is to say that $G$ is obtained from $H$ by gluing a free abelian group $\mathbb{Z}^{n}$ along the (maximal abelian) subgroup $E$ of $H$.

We note that when we say "gluing $\mathbb{Z}^{n}$ along the subgroup $E$ of $H$ ", the outcome will really be the amalgamated free product $\left(E \oplus \mathbb{Z}^{n}\right) *_{E} H$, but we keep this terminology as it will be convenient in what follows.

We observe that if $G$ has the structure of an abelian flat over a subgroup $H$, then it is not hard to find a retraction $r: G \rightarrow H$ : one can use the projection of $E \oplus \mathbb{Z}^{m}$ to $E$ and extend this to a morphism from $G$ to $H$ which fixes $H$.

Example 4.6. The group

$$
G:=\left\langle x_{1}, \ldots, x_{n}, z_{1}, \ldots, z_{k} \mid x_{1}^{2} \cdot \ldots \cdot x_{n}^{2}=1,\left[x_{1}, z_{i}\right],\left[z_{i}, z_{j}\right], i, j \leq k\right\rangle
$$

has the structure of a free abelian flat over the subgroup $\left\langle x_{1}, \ldots, x_{n} \mid x_{1}^{2} \cdot \ldots \cdot x_{n}^{2}\right\rangle$.

Concisely, $G$ is obtained from $\left\langle x_{1}, \ldots, x_{n} \mid x_{1}^{2} \cdots x_{n}^{2}\right\rangle$ by gluing the free abelian group $\mathbb{Z}^{k}$ along the subgroup $\left\langle x_{1}\right\rangle$ of $\left\langle x_{1}, \ldots, x_{n} \mid x_{1}^{2} \cdots x_{n}^{2}\right\rangle$. 
We can combine surface and abelian flats in order to obtain the "floors" of a tower.

Definition 4.7 (Floor). Let $G$ be a group and let $H$ be a subgroup of $G$ (see Figure 4). Then $G$ has the structure of a floor over $H$ if $G$ acts minimally on a tree $T$ and the action admits a Bass-Serre presentation $\left(T^{1}, T^{0},\left\{\gamma_{e}\right\}\right)$, where the set of vertices of $T^{1}$ is partitioned into three subsets, $V_{S}, V_{A}$, and $V_{R}$, such that:

- each vertex in $V_{S}$ is a surface type vertex;

- for each vertex $u \in V_{A}$, its stabilizer $G_{u}$ is a free abelian group;

- the tree $T^{1}$ is bipartite between $V_{S} \cup V_{A}$ and $V_{R}$;

- the subgroup $H$ of $G$ is the free product of the stabilizers of vertices in $V_{R}$;

- for each $u \in V_{A}$, there is a unique edge $e=\{v, u\}$ connecting $u$ to a vertex $v$ in $V_{R}$. Moreover, $\operatorname{Stab}_{G}(e)$ is maximal abelian in $\operatorname{Stab}_{G}(v)$ and $\operatorname{Stab}_{G}(u)=\operatorname{Stab}_{G}(e) \oplus \mathbb{Z}^{m}$. In addition, the stabilizer $\operatorname{Stab}_{G}(e)$ of $e$ cannot be conjugated to any other stabilizer $\operatorname{Stab}_{G}\left(e^{\prime}\right)$ for $e^{\prime} \neq e$ an edge connecting a vertex in $V_{A}$ to a vertex in $V_{R}$;

- either there exists a retraction $r: G \rightarrow H$ that, for each $v \in V_{S}$, sends $\operatorname{Stab}_{G}(v)$ to a nonabelian image or $H$ is cyclic and there exists a retraction $r^{\prime}: G * \mathbb{Z} \rightarrow H * \mathbb{Z}$ that, for each $v \in V_{S}$, sends $\operatorname{Stab}_{G}(v)$ to a nonabelian image.

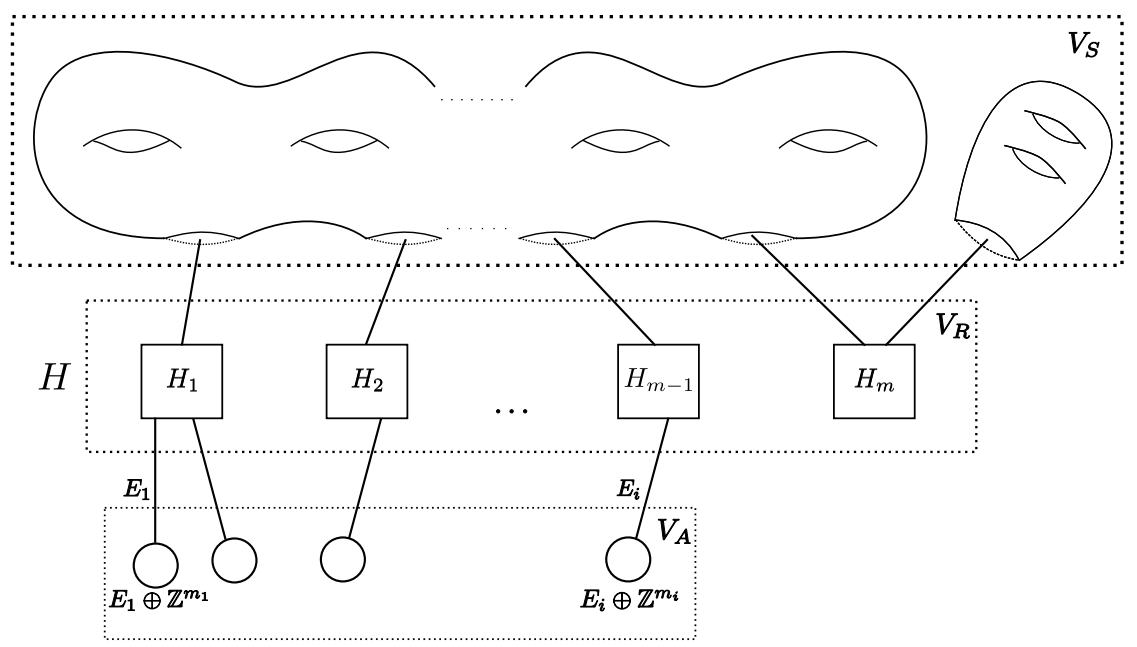

Figure 4. A floor together with the partition of its vertices

In the opposite direction a floor can be decomposed into flats in many possible ways; i.e., a floor can be seen as a sequence of surface and abelian flats, and we will often see such a sequence as giving a preferred order to the flats of the floor.

We can now bring everything together to define:

Definition 4.8. A group $G$ has the structure of a tower (of height $m$ ) over a subgroup $H$ if there exists a sequence $G=G^{m}>G^{m-1}>\cdots>G^{0}=H$ such that for each $i, 0 \leq i<m$, one of the following holds:

(i) The group $G^{i+1}$ has the structure of a floor over $G^{i}$, in which $H$ is contained in one of the vertex groups that generate $G_{i}$ in the floor decomposition of 
$G^{i+1}$ over $G^{i}$. Moreover, the pegs of the abelian flats of the floor are glued along (maximal abelian) groups that are not conjugates of each other and they cannot be conjugated into groups which correspond to abelian flats of any previous floor.

(ii) The group $G^{i+1}$ is a free product of $G^{i}$ with a finitely generated free group.

The next lemma follows from the definition of a constructible limit group.

Lemma 4.9. If $G$ has the structure of a tower over a limit group, then $G$ is a limit group.

If $G$ has the structure of a tower over a subgroup $H$ it will be useful to collect the information witnessing it. Thus we define:

Definition 4.10. Suppose $G$ has the structure of a tower (of height $m$ ) over $H$. Then the tower corresponding to $G$, denoted by $\mathcal{T}(G, H)$, is the following collection of data:

$$
\left(\left(\mathcal{G}\left(G^{1}, G^{0}\right), r_{1}\right),\left(\mathcal{G}\left(G^{2}, G^{1}\right), r_{2}\right), \ldots,\left(\mathcal{G}\left(G^{m}, G^{m-1}\right), r_{m}\right)\right)
$$

where:

- the splitting $\mathcal{G}\left(G^{i+1}, G^{i}\right)$ is the splitting that witnesses that $G^{i+1}$ has the structure of a floor over $G^{i}$, respectively, the free splitting $G^{i} * \mathbb{F}_{n}$ for some finitely generated free group $\mathbb{F}_{n}$;

- the morphism $r_{i+1}: G^{i+1} \rightarrow G^{i}$ (or $r_{i+1}: G^{i+1} \rightarrow G^{i} * \mathbb{Z}$ ) is the retraction that witnesses that $G^{i+1}$ has the structure of a floor over $G^{i}$, respectively, the retraction $r_{i+1}: G^{i} * \mathbb{F}_{n} \rightarrow G^{i}$.

Remark 4.11. The notation $\mathcal{G}(G)$ will refer to a splitting of $G$ as a graph of groups. The notation $\mathcal{G}(G, H)$ will refer either to a free splitting of $G$ as $H * \mathbb{F}_{n}$ or to a splitting that corresponds to a floor structure of $G$ over $H$.

A tower in which no abelian flat occurs in some (any) decomposition of its floors into flats is called a hyperbolic tower (or regular NTQ group in the terminology of Kharlampovich-Myasnikov). Furthermore, if a floor consists only of abelian flats we call it an abelian floor.

For the rest of the paper we assume the following.

Convention. Suppose $\mathcal{T}(G, \mathbb{F})$ is a tower. Let $\left\{E_{j}\right\}_{j \in J}$ be the collection of pegs that correspond to the abelian flats that occur along the floors of the tower. Let $\left\{E_{j}\right\}_{j \in J^{\prime}}$, with $J^{\prime} \subseteq J$, be the subcollection of the pegs that can be conjugated into a subgroup of the base floor $\mathbb{F}$; i.e., there is $\gamma_{j} \in G$ such that $E_{j}^{\gamma_{j}} \leq \mathbb{F}$ for every $j \in J^{\prime}$.

Then, we assume that:

(1) when the above subcollection is not empty, the first floor $\mathcal{G}\left(G^{1}, G^{0}\right)$ of the tower $\mathcal{T}(G, \mathbb{F})$ consists only of the abelian flats corresponding to the above subcollection and glued along $E_{j}^{\gamma_{j}}$ to $\mathbb{F}$, and each floor above the first (abelian) floor is either a free product or it consists of a single flat (abelian or surface);

(2) when the above subcollection is empty, we assume that each floor is either a free product or it consists of a single flat (abelian or surface). 
4.2. Twin towers. We next work towards constructing a tower by "gluing" two copies of a given tower together.

Definition 4.12. Suppose $H$ has the structure of an abelian floor over $\mathbb{F}$ and $\mathcal{G}(H, \mathbb{F})$ is the splitting witnessing it. Let $\left\{\mathbb{Z}^{m_{i}}\right\}_{i \in I}$ be the collection of the free abelian groups that we glue along the corresponding pegs $\left\{E_{i}\right\}_{i \in I}$ in forming the abelian flats of the floor.

Then the double of $H$ with respect to $\mathcal{G}(H, \mathbb{F})$, denoted by $H_{D b}:=H_{D b}(\mathcal{G}(G, \mathbb{F}))$, is the group obtained as the fundamental group of a graph of groups in which all the data is as in $\mathcal{G}(H, \mathbb{F})$ apart from replacing $\left\{\mathbb{Z}^{m_{i}}\right\}_{i \in I}$ by their doubles $\left\{\mathbb{Z}^{m_{i}} \oplus \mathbb{Z}^{m_{i}}\right\}_{i \in I}$. The above graph of groups $D b(\mathcal{G}(H, \mathbb{F})):=\mathcal{G}\left(H_{D b}, \mathbb{F}\right)$ is called the floor double of $\mathcal{G}(H, \mathbb{F})$ and naturally witnesses that $H_{D b}$ is an abelian floor over $\mathbb{F}$ (see Figure 5 ).
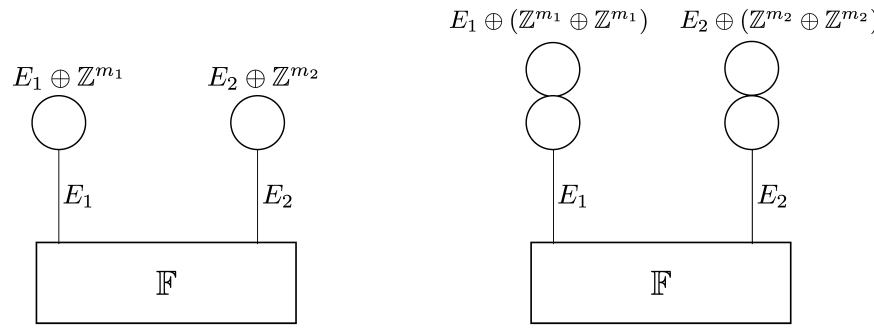

Figure 5. An abelian floor over a free group and its double.

Lemma 4.13. Suppose $H$ has the structure of an abelian floor $\mathcal{G}(H, \mathbb{F})$ over $\mathbb{F}$. Then $H$ admits two natural embeddings $f_{1}, f_{2}$ into $H_{D b}$.

Moreover, for each $i \leq 2$, the group $H_{D b}$ admits an abelian floor structure over $f_{i}(H)$.

Proof. The first embedding $f_{1}$ can be taken to be the identity since $H$ is a subgroup of $H_{D b}$, and clearly $H_{D b}$ has an abelian floor structure over $H$ with the pegs corresponding to the maximal abelian groups of $H$ that contain the pegs of $\mathcal{G}(H, \mathbb{F})$. The second embedding $f_{2}$ is obtained as follows:

- it agrees with Id on $\mathbb{F}$, and

- it sends each free abelian group $\mathbb{Z}^{m}$ that is glued along a peg $E$ in $\mathbb{F}$ in forming the abelian flats of the abelian floor $\mathcal{G}(H, \mathbb{F})$ isomorphically onto the corresponding free abelian group glued along the peg in $H$ that contains $E$ in forming the abelian flats of the abelian floor $\mathcal{G}\left(H_{D b}, H\right)$.

The following lemmata are immediate.

Lemma 4.14. Let $H$ be a subgroup of a group $G$. Suppose $H$ has the structure of an abelian floor $\mathcal{G}(H, \mathbb{F})$ over $\mathbb{F}$. Let $G_{D b}:=H_{D b} *_{H} G$. Let $\mathcal{G}(G)$ be a splitting of $G$ in which $H$ is a subgroup of a vertex group $G_{v}$. Then $G_{D b}$ is isomorphic to the fundamental group of the graph of groups that has the same data as $\mathcal{G}(G)$ apart from replacing $G_{v}$ by $\left(G_{v}\right)_{D b}:=H_{D b} *_{H} G_{v}$.

Lemma 4.15. Suppose $G$ has an abelian floor structure over a limit group $L$. Let $B_{1}, B_{2}$ be nonconjugate (in $L$ ) maximal abelian subgroups of $L$ that cannot be conjugated into any of the pegs of the abelian floor. Then $B_{1}, B_{2}$ are nonconjugate maximal abelian subgroups of $G$. 
We now pass to proving that replacing the first (abelian) floor by its double yields a natural tower structure for the corresponding group.

Lemma 4.16. Suppose $G$ has the structure of a tower (of height $m) \mathcal{T}(G, \mathbb{F}):=$ $\left(\left(\mathcal{G}\left(G^{1}, G^{0}\right), r_{1}\right),\left(\mathcal{G}\left(G^{2}, G^{1}\right), r_{2}\right), \ldots,\left(\mathcal{G}\left(G^{m}, G^{m-1}\right), r_{m}\right)\right)$ over $\mathbb{F}$ and that the first floor $\mathcal{G}\left(G^{1}, G^{0}\right)$ is an abelian floor. Let, for each $i \leq m$, the group $G_{D b}^{i}$ be the amalgamated free product $G_{D b}^{1} *_{G_{1}} G^{i}$, where the edge maps are $f: G_{1} \rightarrow G_{D b}^{1}$ and the embedding induced by the tower structure from $G_{1}$ to $G_{i}$.

Then $G_{D b}:=G_{D b}^{m}$ admits a structure of a tower over $\mathbb{F}$ witnessed by $G_{D b}^{m}>$ $G_{D b}^{m-1}>\cdots>G_{D b}^{1}>\mathbb{F}$ and splittings $\mathcal{G}\left(G_{D b}^{i+1}, G_{D b}^{i}\right)$, which are naturally inherited from the corresponding splittings in $\mathcal{T}(G, \mathbb{F})$.

Proof. The proof is by induction on the height $m$ of the tower.

Base step. The group $G_{D b}^{1}$ has a natural abelian floor structure over $\mathbb{F}$ as observed in Definition 4.12 .

Inductive step. We will assume that the result holds for any tower of height at most $i$ and we show it for towers of height $i+1$. We take cases according to whether $G^{i+1}$ is a free product over $G^{i}$ or has a surface flat structure over $G^{i}$ or has an abelian flat structure over $G^{i}$ :

- Assume that $G^{i+1}=G^{i} * \mathbb{F}_{n}$; then, by Lemma 4.14, $G_{D b}^{i+1}=G_{D b}^{i} * \mathbb{F}_{n}$. So, by the induction hypothesis $G_{D b}^{i+1}$ has a tower structure over $\mathbb{F}$ corresponding naturally to the tower structure of $G^{i+1}$ over $\mathbb{F}$.

- Assume that $G^{i+1}$ has a surface flat structure over $G^{i}$ witnessed by $\left(\mathcal{G}\left(G^{i+1}, G^{i}\right), r_{i+1}\right)$. We consider the rigid vertex group, say $G_{u}$, of the above graph of groups that contains $\mathbb{F}$. Since $G^{1}$ is freely indecomposable with respect to $\mathbb{F}$, the vertex group $G_{u}$ must contain $G^{1}$. We consider the graph of groups with the same data as $\left(\mathcal{G}\left(G^{i+1}, G^{i}\right), r_{i+1}\right)$ apart from replacing $G_{u}$ by $G_{u} *_{G^{1}} G_{D b}^{1}$. Then by Lemma 4.14 the fundamental group of this latter graph of groups is isomorphic to $G_{D b}^{i+1}$, and together with the retraction $r_{i+1}^{\prime}: G_{D b}^{i+1} \rightarrow G_{D b}^{i}$ that agrees with $r_{i+1}$ on $G^{i+1}$ and stays the identity on $G_{D b}^{1}$ it witnesses that $G_{D b}^{i+1}$ has a surface flat structure over $G_{D b}^{i}$.

- Assume that $G^{i+1}$ has an abelian flat structure over $G^{i}$; i.e., $G^{i+1}=G^{i} *_{A}$ $\left(A \oplus \mathbb{Z}^{n}\right)$. We consider the splitting $\left(G^{i} *_{G^{1}} G_{D b}^{1}\right) *_{A}\left(A \oplus \mathbb{Z}^{n}\right)$. By Lemma 4.14 this is a splitting of $G_{D b}^{i+1}$. It is not hard to see that the group $A$ is maximal abelian in $G^{i} *_{G^{1}} G_{D b}^{1}$ : indeed since $A$ cannot be conjugated to any of the pegs of the first abelian floor $\mathcal{G}\left(G^{1}, \mathbb{F}\right)$ and $A$ is maximal abelian in $G^{i}$, it must be maximal abelian in $G_{D b}^{i}$. Moreover, by Lemma 4.15, it cannot be conjugated to any other peg in $G_{D b}^{i}$. Thus, together with the retraction $r_{i+1}^{\prime}: G_{D b}^{i+1} \rightarrow G_{D b}^{i}$ that agrees with $r_{i+1}$ on $G^{i+1}$ and stays the identity on $G_{D b}^{1}$ it witnesses that $G^{i+1}$ has an abelian flat structure over $G_{D b}^{i}$.

Changing slightly the hypothesis of the previous lemma yields the following remark.

Remark 4.17. Suppose $G$ has the structure of a tower (of height $m) \mathcal{T}(G, \mathbb{F}):=$ $\left(\left(\mathcal{G}\left(G^{1}, G^{0}\right), r_{1}\right),\left(\mathcal{G}\left(G^{2}, G^{1}\right), r_{2}\right), \ldots,\left(\mathcal{G}\left(G^{m}, G^{m-1}\right), r_{m}\right)\right)$ over $\mathbb{F}$ and that the first floor $\mathcal{G}\left(G^{1}, G^{0}\right)$ is an abelian floor. Let $f: G^{1} \hookrightarrow G_{D b}^{1}$ be the nonidentity natural 
map from $G^{1}$ to its double (see Lemma 4.13). Let, for each $i \leq m$, the group ${ }_{f} G_{D b}^{i}$ be the amalgamated free product $G_{D b}^{1} *_{f\left(G^{1}\right)} G^{i}$.

Then ${ }_{f} G_{D b}:={ }_{f} G_{D b}^{m}$ admits a structure of a tower over $\mathbb{F}$ witnessed by ${ }_{f} G_{D b}^{m}>$ ${ }_{f} G_{D b}^{m-1}>\cdots>{ }_{f} G_{D b}^{1}>\mathbb{F}$ and splittings $\mathcal{G}\left({ }_{f} G_{D b}^{i+1},{ }_{f} G_{D b}^{i}\right)$, as in Lemma 4.16.

Before moving to the definition of a twin tower we record some easy lemmata that will help us prove that our construction of a twin tower is indeed a tower.

Lemma 4.18. Suppose $G$ has the structure of a tower over a limit group L. Let $E$ be a maximal abelian subgroup of $G$ and suppose $E \cap L$ is not trivial. Let $B$ be the maximal abelian group in $L$ that contains $E \cap L$. Then either $E$ is $B$ or $E$ is the free abelian group $B \oplus \mathbb{Z}^{n}$ that corresponds to an abelian flat of some floor of the tower glued along $B$ to $L$.

The following lemma is an easy exercise in normal forms.

Lemma 4.19. Let $G:=A *_{C} B$ be a limit group and let $E$ be a maximal abelian group in $A$. Suppose that no nontrivial element of $E$ commutes with a nontrivial element of $C$. Then $E$ is maximal abelian in $G$.

We define the notion of a twin tower, first in a case which is free of some technical complexity, in the following proposition.

Proposition 4.20 (Twin tower - nonabelian case). Suppose $G$ has the structure of a tower $\mathcal{T}(G, \mathbb{F})$ over $\mathbb{F}$. Assume that the first floor $\mathcal{G}\left(G^{1}, G^{0}\right)$ is not an abelian floor. Then the amalgamated free product $G *_{\mathbb{F}} G$ admits a natural tower structure over $\mathbb{F}$ which we call the twin tower of $G$ with respect to $\mathcal{T}(G, \mathbb{F})$ (see Figure $[$ ).

Proof. Let $\left(\left(\mathcal{G}\left(G^{1}, G^{0}\right), r_{1}\right), \ldots,\left(\mathcal{G}\left(G^{m}, G^{m-1}\right), r_{m}\right)\right)$ be the sequence witnessing that $G$ is a tower over $\mathbb{F}$. Let $G^{m+i}:=G^{i} *_{\mathbb{F}} G$ be the amalgamated free product of $G^{i}$ with $G$ over $\mathbb{F}$. We claim that there exists a sequence

$$
\begin{array}{r}
\left(\left(\mathcal{G}\left(G^{1}, G^{0}\right), r_{1}\right), \ldots,\left(\mathcal{G}\left(G^{m}, G^{m-1}\right), r_{m}\right),\left(\mathcal{G}\left(G^{m+1}, G^{m}\right), r_{m+1}\right),\right. \\
\left.\ldots,\left(\mathcal{G}\left(G^{2 m}, G^{2 m-1}\right), r_{2 m}\right)\right),
\end{array}
$$

where the splitting $\mathcal{G}\left(G^{m+i+1}, G^{m+i}\right)$ has the same data as the splitting $\mathcal{G}\left(G^{i+1}, G^{i}\right)$, apart from replacing the vertex group $G_{u}$ that contains $\mathbb{F}$ with $G_{u} *_{\mathbb{F}} G$, and moreover it witnesses that $G *_{\mathbb{F}} G$ has the structure of a tower over $\mathbb{F}$. We proceed by induction:

Base step. We show that $G^{m+1}$ is a free product or has a surface flat structure or has an abelian flat structure over $G^{m}=G$, according to whether $G^{1}$ is a free product or has a surface flat structure $\mathbb{F}$. In addition, we show that it respects the requirements of being a floor of a tower together with the already given sequence of floors. We take cases:

- Assume that $G^{1}=\mathbb{F} * \mathbb{F}_{n}$; then $G^{m+1}=\left(\mathbb{F} * \mathbb{F}_{n}\right) *_{\mathbb{F}} G=G * \mathbb{F}_{n}$. Thus, $G^{m+1}$ has a free product structure over $G$.

- Assume that $G^{1}$ has a surface flat structure over $\mathbb{F}$. We consider the graph of groups with the same data as in $\mathcal{G}\left(G^{1}, \mathbb{F}\right)$ apart from replacing the vertex group $\mathbb{F}$ by the amalgamated free product $G *_{\mathbb{F}} \mathbb{F}$. Then the fundamental group of this graph of groups is isomorphic to $G^{m+1}$, and together with the retraction $r_{m+1}: G^{m+1} \rightarrow G^{m}$ that agrees with $r_{1}$ on $G^{1}$ and stays the identity on $G$, it witnesses that $G^{m+1}$ has a surface flat structure over $G^{m}$. 
Inductive step. Assume that the result holds for all $G^{m+1}, \ldots, G^{m+i}$. We will show that it holds for $G^{m+i+1}$. We take cases according to whether $G^{i+1}$ is a free product or has a surface flat structure or an abelian flat structure over $G^{i}$ :

- Assume that $G^{i+1}=G^{i} * \mathbb{F}_{n}$; then $G^{m+i+1}=G^{i+1} *_{\mathbb{F}} G=G^{i} *_{\mathbb{F}} G * \mathbb{F}_{n}=$ $G^{m+i} * \mathbb{F}_{n}$. Thus, $G^{m+i+1}$ is a free product of $G^{m}$ with $\mathbb{F}_{n}$ and it satisfies the conditions of being part of a tower with the already given sequence of floors.

- Assume that $G^{i+1}$ has a surface flat structure $\mathcal{G}\left(G^{i+1}, G^{i}\right)$ over $G^{i}$. Consider the graph of groups decomposition with the same data as in $\mathcal{G}\left(G^{i+1}, G^{i}\right)$, apart from replacing the vertex group $G_{u}$ that contains $\mathbb{F}$ with the amalgamated free product $G_{u} *_{\mathbb{F}} G$. The fundamental group of this graph of groups is isomorphic to $G^{m+i+1}$, and together with the retraction $r_{m+i+1}: G^{m+i+1} \rightarrow G^{m+i}$ that agrees with $r_{i+1}$ on $G^{i+1}$ and stays the identity on $G$, it witnesses that $G^{m+i+1}$ has a surface flat structure over $G^{m+i}$.

- Assume that $G^{i+1}$ has an abelian flat structure $G^{i} *_{A}\left(A \oplus \mathbb{Z}^{n}\right)$ over $G^{i}$. Consider the amalgamated free product $\left(G^{i} *_{\mathbb{F}} G\right) *_{A}\left(A \oplus \mathbb{Z}^{n}\right)$. This is a splitting of $G^{m+i+1}$, and moreover, by Lemma 4.19, $A$ is maximal abelian in $G^{i} *_{\mathbb{F}} G$. A maximal abelian group of $G^{m+i}$ that contains a peg of a previous abelian flat must live either in $G^{i}$ or in $G$. Now it is enough to observe that $A$ cannot be conjugated to a maximal abelian group of $G$ and if a conjugate $A^{g}$ of $A$ intersects nontrivially another maximal abelian group of $G^{i}$, then $g \in G^{i}$. In addition, we define the map $r_{m+i+1}: G^{m+i+1} \rightarrow G^{m+i}$ to agree with $r_{i+1}$ on $G^{i+1}$ and stay the identity on $G$.
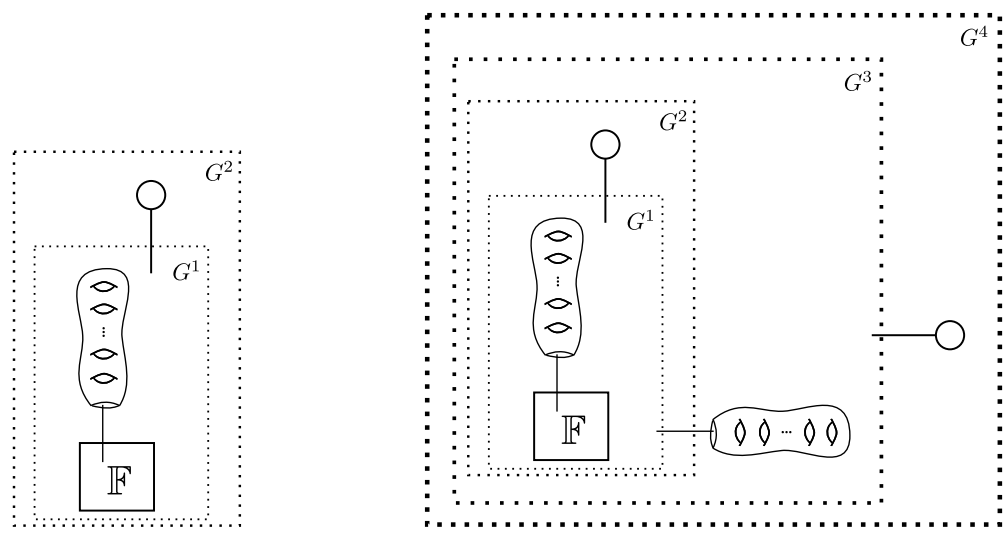

FiguRE 6. A tower and its corresponding twin tower - nonabelian case.

Example 4.21. Let $G:=\left\langle x_{1}, x_{2}, e_{1}, e_{2}, z_{1}, z_{2}\right|\left[x_{1}, x_{2}\right] \cdot\left[e_{1}, e_{2}\right],\left[x_{1}^{3} x_{2}^{4}, z_{1}\right],\left[x_{1}^{3} x_{2}^{4}, z_{1}\right]$, $\left.\left[z_{1}, z_{2}\right]\right\rangle$. We can give $G$ a tower structure over $\mathbb{F}_{2}:=\left\langle e_{1}, e_{2}\right\rangle$ as follows. The tower consists of two floors:

- The first floor is just a surface flat that is obtained by gluing $\Sigma_{1,1}$, whose fundamental group $\pi_{1}\left(\Sigma_{1,1}\right)$ is $\left\langle x_{1}, x_{2}, s \mid s^{-1} \cdot\left[x_{1}, x_{2}\right]\right\rangle$, along its boundary to the subgroup $\left\langle\left[e_{1}, e_{2}\right]\right\rangle$ of the free group $\mathbb{F}_{2}$. In group theoretic terms 
the first floor is the amalgamated free product $G^{1}:=\mathbb{F}_{2} *_{\left[e_{1}, e_{2}\right]=s} \pi_{1}\left(\Sigma_{1,1}\right)$. The retraction $r_{1}$ sends $x_{i}$ to $e_{i}$ and stays the identity on $e_{i}$.

- The second floor is just an abelian flat that is obtained by gluing $\mathbb{Z}^{2}$ along the (maximal) abelian subgroup $\left\langle x_{1}^{3} x_{2}^{4}\right\rangle$ of $G^{1}$. In group theoretic terms the second floor is the amalgamated free product $G^{2}:=G^{1} *_{x_{1}^{3}} x_{2}^{4}=z\left(\langle z\rangle \oplus \mathbb{Z}^{2}\right)$. The retraction $r_{2}$ sends $z_{1}$ and $z_{2}$ to $z$ and stays the identity on $G^{1}$.

The group $G *_{\mathbb{F}_{2}} G$ can be given a twin tower structure as follows. This tower has four floors, which we describe:

- The first two floors are identical to the floors of the tower structure of $G$.

- The third floor is just a surface flat that is obtained by gluing $\Sigma_{1,1}$, whose fundamental group $\pi_{1}\left(\Sigma_{1,1}\right)$ is $\left\langle y_{1}, y_{2}, s \mid s^{-1} \cdot\left[y_{1}, y_{2}\right]\right\rangle$, along its boundary to the subgroup $\left\langle\left[e_{1}, e_{2}\right]\right\rangle$ of the free group $\mathbb{F}_{2}$. In group theoretic terms the third floor is the amalgamated free product $G^{3}:=G^{2} *_{\left[e_{1}, e_{2}\right]=s} \pi_{1}\left(\Sigma_{1,1}\right)$. The retraction $r_{3}$ sends $y_{i}$ to $e_{i}$ and stays the identity on $G^{2}$.

- The fourth floor is just an abelian flat that is obtained by gluing $\mathbb{Z}^{2}$ along the (maximal) abelian subgroup $\left\langle y_{1}^{3} y_{2}^{4}\right\rangle$ of $G^{3}$. In group theoretic terms the fourth floor is the amalgamated free product $G^{4}:=G^{3} *_{1}^{3} y_{2}^{4}=z\left(\langle z\rangle \oplus \mathbb{Z}^{2}\right)$. The retraction $r_{4}$ sends $z_{1}^{\prime}$ and $z_{2}^{\prime}$ to $z$ and stays the identity on $G^{3}$.

Proposition 4.22 (Twin tower - abelian case). Suppose G has the structure of a tower $\mathcal{T}(G, \mathbb{F})$ (of height $m$ ) over $\mathbb{F}$. Assume that the first floor $\mathcal{G}\left(G^{1}, G^{0}\right)$ is an abelian floor (see Figure [7).

Let $G_{D b}^{1}$ be the double of $G^{1}$ with respect to $\mathcal{G}\left(G^{1}, G^{0}\right)$ and let $f: G^{1} \hookrightarrow G_{D b}^{1}$ be the nonidentity natural embedding of $G^{1}$ into $G_{D b}^{1}$.

Let $G_{D b}$ be the double of $G$ with respect to $\mathcal{G}\left(G^{1}, G^{0}\right)$, and let ${ }_{f} G_{D b}$ be the amalgamated free product $G_{D b}^{1} *_{G^{1}} G$, where $f_{\bar{e}}: G^{1} \rightarrow G_{D b}^{1}$ is $f$ and $f_{e}: G^{1} \rightarrow G$ is the identity map. Then the amalgamated free product $G_{D b} *_{G_{D b}^{1}}\left({ }_{f} G_{D b}\right)$ admits a natural tower structure over $\mathbb{F}$.

Proof. Suppose that $\left(\left(\mathcal{G}\left(G^{1}, G^{0}\right), r_{1}\right),\left(\mathcal{G}\left(G^{2}, G^{1}\right), r_{2}\right), \ldots,\left(\mathcal{G}\left(G^{m}, G^{m-1}\right), r_{m}\right)\right)$ witnesses that $G$ has the structure of a tower over $\mathbb{F}$.

Let

$$
\mathcal{T}\left(G_{D b}, \mathbb{F}\right):=\left(\left(\mathcal{G}\left(G_{D b}^{1}, G^{0}\right), r_{1}^{D b}\right),\left(\mathcal{G}\left(G_{D b}^{2}, G_{D b}^{1}\right), r_{2}^{D b}\right), \ldots,\left(\mathcal{G}\left(G_{D b}^{m}, G_{D b}^{m-1}\right), r_{m}^{D b}\right)\right)
$$

be the natural tower structure (see Lemma 4.16) of $G_{D b}$ over $\mathbb{F}$, and let

$$
\mathcal{T}\left({ }_{f} G_{D b}, G_{D b}^{1}\right):=\left(\left(\mathcal{G}\left({ }_{f} G_{D b}^{2}, G_{D b}^{1}\right), r_{2}^{f}\right), \ldots,\left(\mathcal{G}\left({ }_{f} G_{D b}^{m},{ }_{f} G_{D b}^{m-1}\right), r_{m}^{f}\right)\right)
$$

be the natural tower structure (see Remark 4.17) of ${ }_{f} G_{D b}$ over $G_{D b}^{1}$.

For each $i<m$, let $G_{D b}^{m+i}=G_{D b} *_{G_{D b}^{1}}\left({ }_{f} G_{D b}^{i+1}\right)$. We claim that there exists a natural sequence of floors

$$
\begin{aligned}
\left(\left(\mathcal{G}\left(G_{D b}^{1}, G^{0}\right), r_{1}^{D b}\right),\left(\mathcal{G}\left(G_{D b}^{2}, G_{D b}^{1}\right), r_{2}^{D b}\right), \ldots,\left(\mathcal{G}\left(G_{D b}^{m}, G_{D b}^{m-1}\right), r_{m}^{D b}\right)\right. & \\
& \left.\left(\mathcal{G}\left(G_{D b}^{m+1}, G_{D b}^{m}\right), r_{m+1}^{D b}\right), \ldots,\left(\mathcal{G}\left(G^{2 m}, G^{2 m-1}\right), r_{2 m}^{D b}\right)\right)
\end{aligned}
$$

that witnesses that $G_{D b} *_{G_{D b}^{1}}\left({ }_{f} G_{D b}\right)$ has a tower structure over $\mathbb{F}$. We construct 
this sequence starting with the $m$ floors of the tower $\mathcal{T}\left(G_{D b}, \mathbb{F}\right)$ and we proceed recursively as follows:

Base step. We show that $G_{D b}^{m+1}$ is a free product or has a surface flat structure or has an abelian flat structure over $G_{D b}^{m}$ according to whether ${ }_{f} G_{D b}^{2}$ is a free product or has a surface flat structure or has an abelian flat structure over $G_{D b}^{1}$ :

- Assume that ${ }_{f} G_{D b}^{2}=G_{D b}^{1} * \mathbb{F}_{n}$; then $G_{D b}^{m+1}=G_{D b} * G_{D b}^{1}\left(G_{D b}^{1} * \mathbb{F}_{n}\right)=$ $G_{D b} * \mathbb{F}_{n}$.

- Assume that ${ }_{f} G_{D b}^{2}$ has a surface flat structure $\left(\mathcal{G}\left({ }_{f} G_{D b}^{2}, G_{D b}^{1}\right), r_{2}^{f}\right)$ over $G_{D b}^{1}$. We consider the graph of groups with the same data as $\mathcal{G}\left({ }_{f} G_{D b}^{2}, G_{D b}^{1}\right)$ apart from replacing the vertex group $G_{D b}^{1}$ with the group $G_{D b}$. The fundamental group of this latter graph of groups is $G_{D b}^{m+1}$, and together with the retraction $r_{m+1}^{D b}: G_{D b}^{m+1} \rightarrow G_{D b}$ that agrees with $r_{2}^{f}$ on ${ }_{f} G_{D b}^{2}$ and stays the identity on $G_{D b}$, it witnesses that $G_{D b}^{m+1}$ has a surface flat structure over $G_{D b}$.

- Assume that ${ }_{f} G_{D b}^{2}$ has an abelian flat structure $G_{D b}^{1} *_{A}\left(A \oplus \mathbb{Z}^{n}\right)$ over $G_{D b}^{1}$. Consider the amalgamated free product $\left(G_{D b} *_{G_{D b}^{1}} G_{D b}^{1}\right) *_{A}\left(A \oplus \mathbb{Z}^{n}\right)$; this is a splitting of $G_{D b}^{m+1}$. By definition $A$ cannot be conjugated to any other peg of some abelian flat of $\mathcal{T}\left(G_{D b}, \mathbb{F}\right)$; thus it is maximal abelian in $G_{D b}$ and satisfies the properties that make $G_{D b} *_{A}\left(A \oplus \mathbb{Z}^{n}\right)$ the $m+1$-th floor of our tower.

Recursive step. Suppose we have constructed the $m+i$-th floor of the tower. We show that $G_{D b}^{m+i+1}$ is a free product or has a surface flat structure or an abelian flat structure over $G_{D b}^{m+i}$ according to whether ${ }_{f} G_{D b}^{i+2}$ is a free product or has a surface flat structure or an abelian flat structure over ${ }_{f} G_{D b}^{i+1}$ :

- Assume that ${ }_{f} G_{D b}^{i+2}={ }_{f} G_{D b}^{i+1} * \mathbb{F}_{n}$; then $G_{D b}^{m+1}=G_{D b} * G_{D b}^{1}\left(G_{D b}^{i+1} * \mathbb{F}_{n}\right)=$ $G_{D b}^{m+i} * \mathbb{F}_{n}$.

- Assume that ${ }_{f} G_{D b}^{i+2}$ has a surface flat structure $\left(\mathcal{G}\left({ }_{f} G_{D b}^{i+2},{ }_{f} G_{D b}^{i+1}\right), r_{i+2}^{f}\right)$ over ${ }_{f} G_{D b}^{i+1}$. We consider the graph of groups with the same data as $\mathcal{G}\left({ }_{f} G_{D b}^{i+2}, G_{D b}^{i+1}\right)$, apart from replacing the vertex group $G_{u}$ that contains $G_{D b}^{1}$ with the group $G_{D b} *_{G_{D b}^{1}} G_{u}$. The fundamental group of this latter graph of groups is $G_{D b}^{m+i+1}$, and together with the retraction $r_{m+i+1}^{D b}$ : $G_{D b}^{m+i+1} \rightarrow G_{D b}^{m+i}$ that agrees with $r_{i+2}^{f}$ on ${ }_{f} G_{D b}^{i+2}$ and stays the identity on $G_{D b}$, it witnesses that $G_{D b}^{m+i+1}$ has a surface flat structure over $G_{D b}^{m+i}$.

- Assume that ${ }_{f} G_{D b}^{i+2}$ has an abelian flat structure ${ }_{f} G_{D b}^{i+1} *_{A}\left(A \oplus \mathbb{Z}^{n}\right)$ over ${ }_{f} G_{D b}^{i+1}$. We consider the amalgamated free product $\left(G_{D b} *_{G_{D b}^{1}}\left({ }_{f} G_{D b}^{i+1}\right)\right) *_{A}$ $\left(A \oplus \mathbb{Z}^{n}\right)$; this is a splitting of $G_{D b}^{m+i+1}$. Since $A$ cannot be conjugated to any other previous peg of the tower $\mathcal{T}\left(G_{D b}, \mathbb{F}\right)$ and the tower $\mathcal{T}\left({ }_{f} G_{D b}, G_{D b}^{1}\right)$, we see that $A$ is maximal abelian in $G_{D b} *_{G_{D b}^{1}}\left({ }_{f} G_{D b}^{i+1}\right)$ and it satisfies the properties that make $G_{D b}^{m+i} *_{A}\left(A \oplus \mathbb{Z}^{n}\right)$ the $m+i+1$-th floor of our tower.

Example 4.23. Let $G:=\left\langle e_{1}, e_{2}, z_{1}, z_{2}, x_{1}, x_{2}\right|\left[z_{1}, z_{2}\right],\left[z_{1}, e_{1}^{2} e_{2}^{2}\right],\left[z_{2}, e_{1}^{2} e_{2}^{2}\right],\left[x_{1}, x_{2}\right]$. $\left.\left[z_{1}, e_{1}\right]\right\rangle$. We can give $G$ a tower structure over $\mathbb{F}_{2}:=\left\langle e_{1}, e_{2}\right\rangle$ as follows. The tower $\mathcal{T}\left(G, \mathbb{F}_{2}\right)$ consists of two floors:

- The first floor is just an abelian flat obtained by gluing $\mathbb{Z}^{2}$ along the maximal abelian group $\left\langle e_{1}^{2} e_{2}^{2}\right\rangle$ of $\mathbb{F}_{2}$. In group theoretic terms, the group 
corresponding to the first floor is the amalgamated free product $G^{1}:=$ $\mathbb{F}_{2} * e_{1}^{2} e_{2}^{2}=z\left(\langle z\rangle \oplus \mathbb{Z}^{2}\right)$. The retraction $r_{1}: G^{1} \rightarrow \mathbb{F}_{2}$ sends $z_{1}$ and $z_{2}$ to $z$ and stays the identity on $\mathbb{F}_{2}$.

- The second floor is just a surface flat obtained by gluing $\Sigma_{1,1}$, whose fundamental group is $\left\langle x_{1}, x_{2}, s \mid s^{-1}\left[x_{1}, x_{2}\right]\right\rangle$, along its boundary to the subgroup $\left\langle\left[z_{1}, e_{1}\right]\right\rangle$ of $G^{1}$. In group theoretic terms the group corresponding to the second floor is the amalgamated free product $G^{2}:=G^{1} *_{\left[z_{1}, e_{1}\right]=s} \pi_{1}\left(\Sigma_{1,1}\right)$. The retraction $r_{2}: G^{2} \rightarrow G^{1}$ sends $x_{1}$ to $z_{1}, x_{2}$ to $e_{1}$, and stays the identity on $G^{1}$.

We now consider the double of $G^{1}$ with respect to the splitting $\mathcal{G}\left(G^{1}, \mathbb{F}\right)$ of the first point above. As a group $G_{D b}^{1}$ has the following presentation:

$$
\left\langle e_{1}, e_{2}, z_{1}, z_{2}, y_{1}, y_{2} \mid\left[z_{i}, y_{j}\right] i, j \leq 2,\left[z_{1}, e_{1}^{2} e_{2}^{2}\right],\left[z_{2}, e_{1}^{2} e_{2}^{2}\right],\left[y_{1}, e_{1}^{2} e_{2}^{2}\right],\left[y_{2}, e_{1}^{2} e_{2}^{2}\right]\right\rangle \text {. }
$$

It can be seen as the amalgamated free product $G_{D b}^{1}:=\mathbb{F}_{2} *_{e_{1}^{2}} e_{2}^{2}=z\left(\langle z\rangle \oplus \mathbb{Z}^{4}\right)$.

The twin tower that corresponds to $\mathcal{T}\left(G, \mathbb{F}_{2}\right)$ consists of three floors as follows:

- The first floor is the floor double of $\mathcal{G}\left(G^{1}, \mathbb{F}_{2}\right)$, and the group corresponding to this floor is $G_{D b}^{1}$. The retraction $r_{1}^{D b}$ sends each $z_{1}, z_{2}, y_{1}, y_{2}$ to $z$ and stays the identity on $\mathbb{F}_{2}$.

- The second floor is just a surface flat obtained by gluing $\Sigma_{1,1}$, whose fundamental group is $\left\langle x_{1}, x_{2}, s \mid s^{-1}\left[x_{1}, x_{2}\right]\right\rangle$, along its boundary to the subgroup $\left\langle\left[z_{1}, e_{1}\right]\right\rangle$ of $G_{D b}^{1}$. In group theoretic terms the group corresponding to the second floor is the amalgamated free product $G_{D b}^{2}:=G_{D b}^{1}{ }^{*}\left[z_{1}, e_{1}\right]=s \pi_{1}\left(\Sigma_{1,1}\right)$. The retraction $r_{2}^{D b}: G_{D b}^{2} \rightarrow G_{D b}^{1}$ sends $x_{1}$ to $z_{1}, x_{2}$ to $e_{1}$, and stays the identity on $G_{D b}^{1}$.

- The third floor is again a surface flat obtained by gluing $\Sigma_{1,1}$, whose fundamental group is $\left\langle p_{1}, p_{2}, s \mid s^{-1}\left[p_{1}, p_{2}\right]\right\rangle$, along its boundary to the subgroup $\left\langle\left[y_{1}, e_{1}\right]\right\rangle$ of $G_{D b}^{2}$. In group theoretic terms the group corresponding to the second floor is the amalgamated free product $G_{D b}^{3}:=G_{D b}^{2}{ }^{*}\left[y_{1}, e_{1}\right]=s \pi_{1}\left(\Sigma_{1,1}\right)$. The retraction $r_{3}^{D b}: G_{D b}^{3} \rightarrow G_{D b}^{2}$ sends $p_{1}$ to $y_{1}, p_{2}$ to $e_{1}$, and stays the identity on $G_{D b}^{2}$.

The group corresponding to the twin tower has presentation

$$
\begin{array}{r}
\left\langle e_{1}, e_{2}, z_{1}, z_{2}, y_{1}, y_{2}, x_{1}, x_{2}, p_{1}, p_{2}\right|\left[z_{i}, y_{j}\right] i, j \leq 2,\left[z_{1}, e_{1}^{2} e_{2}^{2}\right],\left[z_{2}, e_{1}^{2} e_{2}^{2}\right],\left[y_{1}, e_{1}^{2} e_{2}^{2}\right], \\
\left.\left[y_{2}, e_{1}^{2} e_{2}^{2}\right],\left[x_{1}, x_{2}\right] \cdot\left[z_{1}, e_{1}\right],\left[p_{1}, p_{2}\right] \cdot\left[y_{1}, e_{1}\right]\right\rangle .
\end{array}
$$

4.3. Closures of towers. We pass to the notion of a tower closure. We first define the notion of an abelian floor closure.

Definition 4.24 (Abelian floor closure). Suppose $G$ has the structure of an abelian floor over a limit group $L$ and $\mathcal{G}(G, L)$ is the splitting witnessing it. Let $\left\{\mathbb{Z}^{m_{i}}\right\}_{i \in I}$ be the collection of the free abelian groups that we glue along the corresponding pegs $\left\{E_{i}\right\}_{i \in I}$ in forming the abelian flats of the floor.

Let $\left\{A^{m_{i}}\right\}_{i \in I}$ be free abelian groups and let $f_{i}: E_{i} \oplus \mathbb{Z}^{m_{i}} \rightarrow E_{i} \oplus A^{m_{i}}$ be an embedding with $f_{i} \uparrow E_{i}=\mathrm{Id}$ and such that $f_{i}\left(E_{i} \oplus \mathbb{Z}^{m_{i}}\right)$ is a finite index subgroup of $E_{i} \oplus A^{m_{i}}$ for every $i \in I$. We call $\left\{f_{i}\right\}_{i \in I}$ a family of closure embeddings.

We denote by $\operatorname{cl}(G)$ the group that is the fundamental group of a graph of groups in which all the data is as in $\mathcal{G}(G, L)$ apart from replacing $\left\{\mathbb{Z}^{m_{i}}\right\}_{i \in I}$ by $\left\{\mathbb{Z}^{m_{i}} \oplus A^{m_{i}}\right\}_{i \in I}$ and adding to the vertex groups $\left\{E_{i} \oplus \mathbb{Z}^{m_{i}} \oplus A^{m_{i}}\right\}_{i \in I}$ the relations 

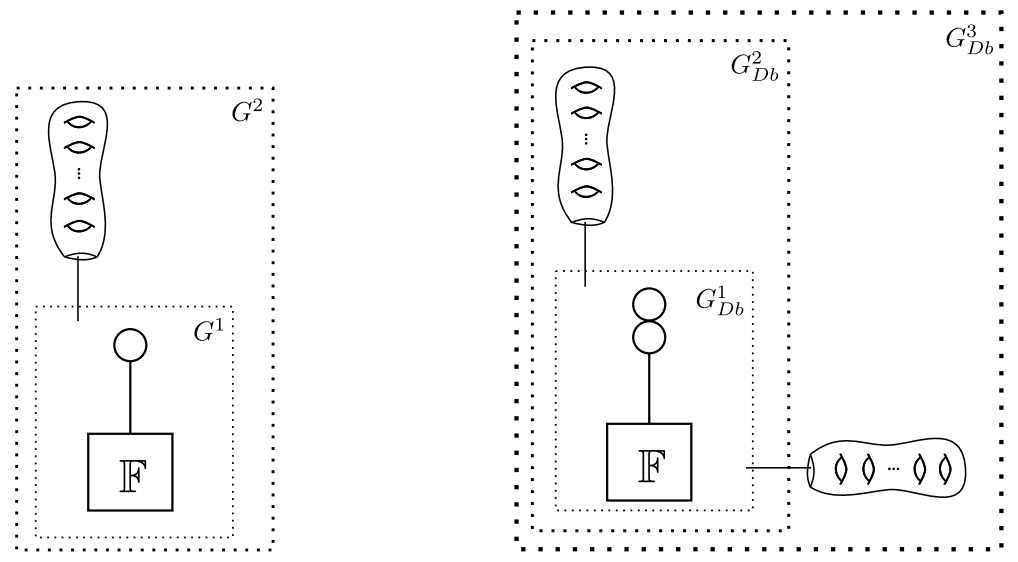

FiguRE 7. A tower and its corresponding twin tower - abelian case.

corresponding to the family of closure embeddings; i.e., $z=f_{i}(z)$ for every $z \in$ $E \oplus \mathbb{Z}^{m_{i}}$. Moreover, we call the latter graph of groups, $\mathcal{G}(\operatorname{cl}(G), L)$, the floor closure of $\mathcal{G}(G, L)$ with respect to $\left\{f_{i}\right\}_{i \in I}$.

In the following paragraph we identify closure embeddings with finite-index subgroups of a "formal" free abelian group $\mathbb{A}^{m}:=\langle(1,0, \ldots, 0), \ldots,(0,0, \ldots, 1)\rangle$. Such an identification will be of use when we wish to understand when a homomorphism from a tower $h: G \rightarrow \mathbb{F}$ extends to $c l(G)$. For simplicity and in accordance with our convention on tower structures, we phrase it for an abelian flat over $\mathbb{F}$, but it holds for an arbitrary tower.

Remark 4.25. Suppose $G$ has the structure of an abelian flat over $\mathbb{F}$, so $G$ is isomorphic to the amalgamated product of $\mathbb{F}$ with $\langle c\rangle \oplus \mathbb{Z}^{m}$ over $c$. Let $\gamma$ generate the centraliser of (the image of) $c$ in $\mathbb{F}$, so that $c=\gamma^{l}$ for some $l$, and let $f: \mathbb{Z}^{m} \rightarrow A^{m}$ be a closure embedding. For each $i \leq m, f\left(z_{i}\right)=c^{k_{i, 0}} a_{1}^{k_{i, 1}} \cdots a_{m}^{k_{i, m}}$ for some $k_{i, j} \in \mathbb{Z}$. Denote by $K=\left(k_{i, j}\right)_{1 \leq i, j \leq m}$ the coefficient matrix. Since the image of $f$ is of finite index in $\mathbb{A}^{m}$, det $K \neq 0$, and so the image of $K$ (considered as a linear transformation on $\mathbb{A}^{m}$ ) is of finite index in $\mathbb{A}^{m}$.

Now let $h: G \rightarrow \mathbb{F}$ be a morphism which restricts to the identity on $\mathbb{F}$. Then $h$ sends each $z_{i}$ to some power $x_{i}$ of $\gamma$, and we can assign to $h$ the vector $\left(x_{1}, \ldots, x_{m}\right)$. If $h$ extends to a morphism $H: \operatorname{cl}(G) \rightarrow \mathbb{F}$, then there are integers $\left(y_{1}, \ldots, y_{m}\right)$ such that $H\left(a_{i}\right)=\gamma^{y_{i}}$ and $x_{i}=k_{i, 0} l+k_{i, 1} y_{1}+\cdots+k_{i, m} y_{m}$. Clearly, the existence of such $y_{i}$ 's is also a sufficient condition for $h$ to extend, so $h$ extends to $\operatorname{cl}(G)$ if and only if $\left(x_{i}-l \cdot k_{i, 0}\right)_{i=1}^{m}$ belongs to $\operatorname{Im}(K)$ or $\left(x_{i}, \ldots, x_{m}\right)$ belongs to $\left(l \cdot k_{1,0}, \ldots, l\right.$. $\left.k_{m, 0}\right)+\operatorname{Im}(K)$.

Example 4.26. Consider the amalgamated product $G=\mathbb{F}_{2} *_{e_{1}^{5}=c}\left(\langle c\rangle \oplus \mathbb{Z}^{2}\right)$. This group admits the structure of an abelian flat over $\mathbb{F}_{2}:=\left\langle e_{1}, e_{2}\right\rangle$.

Let $f:\langle c\rangle \oplus \mathbb{Z}^{2} \rightarrow\langle c\rangle \oplus A^{2}$ be the morphism that restricts to the identity on $c$ and sends $z_{1}$ to $c^{2} a_{1}^{3} a_{2}$ and $z_{2}$ to $a_{1} a_{2}$. This is a closure embedding, and $c l(G)$ is the amalgamated product $c l(G)=\mathbb{F}_{2} *_{e_{1}^{5}=c}\left(\langle c\rangle \oplus \mathbb{Z}^{2} \oplus A^{2} /\left\langle z_{1}=c^{2} a_{1}^{3} a_{2}, z_{2}=a_{1} a_{2}\right\rangle\right)$. 
A morphism $h: G \rightarrow \mathbb{F}_{2}$ (that restricts to the identity on $\mathbb{F}_{2}$ ) satisfies $h\left(z_{i}\right)=e_{1}^{x_{i}}$ for $i=1,2$. It extends to a morphism $H: \operatorname{cl}(G) \rightarrow \mathbb{F}_{2}$ if and only if $x_{1}=$ $5 l+3 y_{1}+y_{2}$ and $x_{2}=y_{1}+y_{2}$ for some $y_{1}, y_{2} \in \mathbb{Z}$. Such $y_{1}, y_{2}$ exist if and only if $\left(x_{1}-5 l, x_{2}\right)=y_{1}(3,1)+y_{2}(1,1)$; that is, $\left(x_{1}, x_{2}\right)$ belongs to the coset [5l,0] of the image of $K=\left(\begin{array}{ll}3 & 1 \\ 1 & 1\end{array}\right)$.

Remark 4.27. Suppose $G$ has the structure of a floor over a limit group $L$. Suppose $\left\{\mathbb{Z}^{m_{i}}\right\}_{i \in I}$ is the collection of the free abelian groups that we glue along the corresponding pegs $\left\{E_{i}\right\}_{i \in I}$ in forming the abelian flats of the floor. Let $\left(\mathcal{G}(\operatorname{cl}(G), L),\left\{f_{i}\right\}_{i} \in I\right)$ be the closure of $G$ with respect to some family of closure embeddings. Let $G=G^{1} * \cdots * G^{m}$ be a free splitting of $G$. Then for each $i \in I$ there exists $\gamma_{i} \in G$ so that $\gamma_{i} E_{i} \oplus \mathbb{Z}^{m_{i}} \gamma_{i}^{-1}$ is a subgroup of some $G_{j}$ for $j \leq m$.

Thus, there exists a free splitting, $H^{1} * \cdots * H^{m}$, of $\operatorname{cl}(G)$ such that each $H^{i}$ is the group obtained by gluing $\gamma_{i} A^{m_{i}} \gamma_{i}^{-1}$ in $G^{i}$ along each maximal abelian group of the form $\gamma_{i} E_{i} \oplus \mathbb{Z}^{m_{i}} \gamma_{i}^{-1}$ that is contained in $G^{i}$ and adding the relations in this new vertex group according to the family of the closure embeddings; i.e., $\gamma_{i} z \gamma_{i}^{-1}=$ $\gamma_{i} f_{i}(z) \gamma_{i}^{-1}$ for every $z \in E_{i} \oplus \mathbb{Z}^{m_{i}}$.

Definition 4.28 (Tower closure). Let $\mathcal{T}(G, \mathbb{F})$ be a tower of height $m$. Let, for $i<m, \mathcal{G}\left(G^{i+1}, G^{i}\right)$ be the $i$-th floor, and let $\left\{f_{j}^{i}\right\}_{j \in J}$ be a family of closure embeddings (in the case where the $i$-th floor is a free product or a hyperbolic floor we take the family to be empty). Then the tower closure, $\operatorname{cl}(\mathcal{T}(G, \mathbb{F}))$, of the tower $\mathcal{T}(G, \mathbb{F})$ with respect to the previous families of closure embeddings for each floor of the tower is defined recursively as follows.

Base step. The first floor consists of the floor closure $\mathcal{G}\left(\operatorname{cl}\left(G^{1}\right), \mathbb{F}\right)$ with respect to $\left\{f_{j}^{1}\right\}_{j \in J}$.

Recursive step. Let $G_{c l}^{i}$ be the group that corresponds to the $i$-th floor of the tower closure.Then $G_{c l}^{i+1}$ is the fundamental group of the graph of groups in which:

- the underlying graph is the same as the underlying graph of $\mathcal{G}\left(G^{i+1}, G^{i}\right)$;

- the vertex groups $G_{1}^{i}, \ldots, G_{m}^{i}$ whose free product is $G^{i}$ are replaced by the corresponding groups in $G_{c l}^{i}$;

- the abelian flats $\mathbb{Z}^{m_{j}}$ are replaced by $A^{m_{j}} \oplus \mathbb{Z}^{m_{j}}$; and

- in the new abelian vertex groups $E_{j} \oplus A^{m_{j}} \oplus \mathbb{Z}^{m_{j}}$ we add relations according to the family of closure embeddings; i.e., $z=f_{j}^{i+1}(z)$ for every $z \in E_{j} \oplus \mathbb{Z}^{m_{j}}$.

It is not hard to see the following.

Lemma 4.29. Let $\mathcal{T}(G, \mathbb{F})$ be a tower over $\mathbb{F}$. Then $\operatorname{cl}(\mathcal{T}(G, \mathbb{F}))$ with respect to any families of closure embeddings is a tower over $\mathbb{F}$.

4.4. Symmetrizing closures of twin towers. When moving to the closure of a twin tower it could be the case that "twin" abelian flats that appear in the floors of the twin tower embed in different ways in the ambient free abelian groups. For example, if $E \oplus \mathbb{Z}^{2}$ is an abelian flat generated by $z_{1}, z_{2}$ and $\hat{E} \oplus \hat{\mathbb{Z}}^{2}$ is its twin generated by $\hat{z_{1}}, \hat{z_{2}}$, then we could have the closure embeddings $f: E \oplus \mathbb{Z}^{2} \rightarrow$ $E \oplus \mathbb{A}^{2}$ which correspond to $(1,2)+\operatorname{Im}(K), K=\left(\begin{array}{ll}3 & 1 \\ 1 & 1\end{array}\right)$ and $\hat{f}: \hat{E} \oplus \hat{\mathbb{Z}}^{2} \rightarrow \hat{E} \oplus \hat{\mathbb{A}}^{2}$ which correspond to $(3,4)+\operatorname{Im}(\hat{K}), \hat{K}=\left(\begin{array}{ll}4 & 5 \\ 1 & 3\end{array}\right)$. We would like the images of $z_{i}, \hat{z}_{i}$ under $f, \hat{f}$ to correspond to the same words in the generators of a closure. This is achieved by closure-embedding $\mathbb{A}^{2}, \hat{\mathbb{A}}^{2}$ in $\mathbb{B}^{2}, \hat{\mathbb{B}}^{2}$ with corresponding closureembeddings $g, \hat{g}$ with coefficient matrices $L, \hat{L}$ such that $K L=\hat{K} \hat{L}$. In this case, 
the closure embedding $g \circ f$ corresponds to $(1,2)+\operatorname{Im}(K L)$, and $\hat{g} \circ \hat{f}$ corresponds to $(3,4)+\operatorname{Im}(K L)$. Both columns of $K L=\hat{K} \hat{L}$ are in the image of $K$, so finding $L, \hat{L}$ comes down to finding two vectors in the images of both $K$ and $\hat{K}$, which are linearly independent. This can be done, since $\operatorname{Im}(K), \operatorname{Im}(\hat{K})$ are of finite index in $\mathbb{A}^{2}$, and so is their intersection.

We define the symmetric closure of a closure of a twin tower as follows.

Definition 4.30. Suppose $G$ has the structure of a tower (of height $m$ ) $\mathcal{T}(G, \mathbb{F}$ ) and that the first floor $\mathcal{G}\left(G^{1}, G^{0}\right)$ is an abelian floor. Enumerate the abelian flats in $G: E_{1} \oplus \mathbb{Z}_{1}^{m_{1}}, \ldots, E_{s} \oplus \mathbb{Z}_{s}^{m_{s}}$. Let $\mathcal{T} \# \mathcal{T}(G, \mathbb{F})$ be the twin tower of $\mathcal{T}(G, \mathbb{F})$. For each $1<i \leq s$, let $\hat{E}_{i} \oplus \mathbb{Z}^{\hat{m}_{i}}$ be the "twin" of the $i$-th flat.

Let $c l(\mathcal{T} \# \mathcal{T}(G, \mathbb{F}))$ be a closure with respect to some closure embeddings $\left\{\left(f_{i}, \hat{f}_{i}\right)_{1 \leq i \leq s}\right\}$, where $f_{i}, \hat{f}_{i}$ are defined on $E_{1} \oplus \mathbb{Z}_{i}^{m_{i}}, \hat{E}_{i} \oplus \mathbb{Z}^{\hat{m}_{i}}$, respectively, and correspond to $p_{i}+\operatorname{Im}\left(K_{i}\right), \hat{p_{i}}+\operatorname{Im}\left(\hat{K}_{i}\right)$. Let $L_{i}, \hat{L}_{i} \in M_{m_{i}}(\mathbb{Z})$ be such that $K_{i} L_{i}=$ $\hat{K}_{i} \hat{L}_{i}$ for each $i$. Let $\left\{g_{i}, \hat{g}_{i}\right\}_{1 \leq i \leq s}$ be the closure embedding corresponding to $p_{i}+\operatorname{Im}\left(K_{i} L_{i}\right), \hat{p}_{i}+\operatorname{Im}\left(K_{i} L_{i}\right)$ for each $i$. Then $\left\{g_{i}, \hat{g}_{i}\right\}_{1 \leq i \leq s}$ is called the symmetric closure embedding of $\left\{f_{i}, \hat{f}_{i}\right\}_{1 \leq i \leq s}$.

Lemma 4.31. The symmetric closure of the closure of a twin tower is a closure of its closure. Moreover, a homomorphism $h: G \rightarrow \mathbb{F}$ of a twin tower extends to the symmetric closure if and only if for each $i$ the vector $\left(x_{1}, \ldots, x_{m_{i}}\right)$ corresponding to $h \uparrow \mathbb{Z}^{m_{i}}$ is in $p_{i}+\operatorname{Im}\left(K_{i} L_{i}\right)$ (respectively, $h \uparrow \mathbb{Z}^{\hat{m}_{i}}$ is in $\hat{p}_{i}+\operatorname{Im}\left(K_{i} L_{i}\right)$ ). As a consequence, if $h: G \rightarrow \mathbb{F}$ extends to a closure of $G$, it also extends to its symmetric closure.

\section{SOLID LIMIT GROUPS AND STRICTLY SOLID MORPHISMS}

In this section we record the definitions of a strictly solid morphism and a family of such morphism as given by Sela. A strictly solid morphism is a morphism from a solid limit group to a free group that satisfies certain conditions. These morphisms are of fundamental importance in the work of Sela in answering Tarski's question: first because of a boundedness result (see [17, Theorem 2.9]) and second because in contrast to solid morphisms they are first order definable.

The definition of a strictly solid morphism requires a technical construction, called the completion of a strict map. The next subsection explains this construction.

In subsection 5.2 we define the above special class of morphisms and their families.

5.1. Completions. We start by modifying a GAD for a limit group $G$ in order to simplify the conditions for a map $\eta: G \rightarrow L$ to be strict with respect to it. The goal is to transform the GAD in a way that the rigid vertex groups will be enlarged to their envelopes, every edge group connecting two rigid vertex groups will be maximal abelian in both vertex groups of the one edged splitting induced by its edge (and after replacing all abelian vertex groups with their peripheral subgroups), and abelian vertex groups will be leaves connected through a rigid vertex to the rest of the graph.

The following lemma of Sela will be helpful. 
Lemma 5.1 (Sela). Let $L$ be a limit group and let $M$ be a noncyclic maximal abelian subgroup. Then:

- if $L$ admits an amalgamated free product splitting with abelian edge group, then $M$ can be conjugated into one of the factors;

- if $L$ admits an $H N N$ extension splitting $A *_{C}$ where $C$ is abelian, then either $M$ can be conjugated into $A$ or $M$ can be conjugated in $\langle C, t\rangle$ and $L=A *_{C}\langle C, t\rangle$.

Lemma 5.2. Let $G$ be a limit group and let $\Delta:=\left(\mathcal{G}(V, E),\left(V_{S}, V_{A}, V_{R}\right)\right)$ be a $G A D$ for $G$, where the image of each edge group is maximal abelian in at least one vertex group of the one edged splitting induced by the edge. Assume moreover that $V_{A}$ is empty. Then there exists a $G A D \hat{\Delta}:=\left(\hat{\mathcal{G}}(\hat{V}, \hat{E}),\left(\hat{V}_{S}, \hat{V}_{A}, \hat{V}_{R}\right)\right)$ for $G$ satisfying the following properties:

(1) the underlying graph $\hat{\mathcal{G}}$ is the same as $\mathcal{G}$ up to some sliding of edges;

(2) the set of rigid vertices and the set of surface type vertices are the same;

(3) every rigid vertex group of the graph of groups $\hat{\mathcal{G}}$ coincides with the envelope of the corresponding rigid vertex group in $\mathcal{G}$;

(4) the image of every edge group connecting two rigid vertices of $\hat{V}_{R}$ is maximal abelian in both vertex groups in the splitting induced by the edge.

Remark 5.3. Suppose $\eta: G \rightarrow L$ is a strict map with respect to the GAD $\Delta$ for $G$. We consider the following modification of $\Delta$ :

Step 1. We replace every vertex group $G_{u}$ with $u \in V_{A}$ by its peripheral subgroup and we place $u$ in the set $V_{R}$ (i.e., we consider it rigid); we call this GAD $\Delta_{0}$.

Step 2. We modify $\Delta_{0}$ according to Lemma 5.2 in order to obtain $\hat{\Delta}_{0}$.

Step 3. To every rigid vertex in $\hat{\Delta}_{0}$ whose vertex group was a peripheral subgroup in $\Delta_{0}$ we attach an edge whose edge group is the peripheral subgroup itself, and the vertex group on its other end is the abelian group that contained the peripheral subgroup in $\Delta$. These new vertex groups will be abelian type vertex groups. We denote by $\hat{\Delta}$ this GAD for $G$.

We will either explicitly or implicitly use the above modification for the rest of the paper.

Definition 5.4. Let $G$ be a group and let $\mathcal{G}(G)$ be a GAD for $G$ with $m$ edges and at least one rigid vertex. Let $\eta: G \rightarrow H$ and let $(\mathcal{G}(G), \eta)$ be strict.

Let $\mathcal{G}_{0} \subseteq \mathcal{G}_{1} \subseteq \cdots \subseteq \mathcal{G}_{m}:=\mathcal{G}(G)$ be a sequence of subgraphs of groups such that $\mathcal{G}_{i}$ has $i$ edges. We define the group $C_{\text {omp }}$ together with its splitting $\mathcal{G}\left(\mathrm{Comp}_{i}\right)$ by the following recursion:

Base step. The subgraph of groups $\mathcal{G}_{0}$ consists of a single vertex $V$ which we may assume is rigid. Then $C o m p_{0}$ is the group $H$ and $\mathcal{G}\left(C o m p_{0}\right)$ is the trivial splitting.

Recursive step. Let $e_{i+1}$ be the edge in $\mathcal{G}_{i+1} \backslash \mathcal{G}_{i}$. We take cases:

Case 1. Suppose $e_{i+1}$ connects two rigid vertices. We further take cases:

1A. Assume that the centralizer of $\eta\left(G_{e_{i+1}}\right)$ in $C o m p_{i}$ cannot be conjugated either to the centralizer of $\eta\left(G_{e}\right)$ for some edge $e$ in $\mathcal{G}_{i}$ that connects two rigid vertex groups or to the centralizer of the image of the peripheral subgroup of some abelian vertex group in $\mathcal{G}_{i}$. Then $C o m p_{i+1}$ is the fundamental group of the graph of groups obtained by gluing to $\mathcal{G}\left(\mathrm{Comp}_{i}\right)$ a free abelian flat 
of rank 1 along the centralizer of $\eta\left(G_{e_{i+1}}\right)$ in $C o m p_{i}$. The latter graph of groups is $\mathcal{G}\left(\operatorname{Comp}_{i+1}\right)$.

1B. Assume that the centralizer of $\eta\left(G_{e_{i+1}}\right)$ in $C_{o m p}$ can be conjugated to the centralizer $C$ (in $C o m p_{i}$ ) either of $\eta\left(G_{e}\right)$ for some edge $e$ in $\mathcal{G}_{i}$ that connects two rigid vertex groups or of the image of the peripheral group of some abelian vertex group (i.e., a vertex group whose vertex belongs to $\left.V_{A}\right)$ in $\mathcal{G}_{i}$. Then $C_{o m p}$ is the fundamental group of the graph of groups obtained by gluing to $\mathcal{G}\left(C o m p_{i}\right)$ a free abelian flat of rank 1 along $C$.

Case 2. Suppose $e_{i+1}$ connects a rigid vertex with a free abelian vertex group of rank $n$ and let $P$ be its peripheral subgroup. We may assume that the free abelian vertex group is not in $\mathcal{G}_{i}$ and we further take cases:

2A. Assume that $\eta(P)$ cannot be conjugated either to the centralizer of $\eta\left(G_{e}\right)$ for some edge $e$ in $\mathcal{G}_{i}$ that connects two rigid vertex groups or to the centralizer of the image of the peripheral subgroup of some abelian vertex group in $\mathcal{G}_{i}$. Then $C_{o m p} p_{i+1}$ is the fundamental group of the graph of groups obtained by gluing to $\mathcal{G}\left(C o m p_{i}\right)$ a free abelian flat of rank $n$ along the centralizer of $\eta(P)$ in $C o m p_{i}$, and moreover in the new abelian vertex group we add the relations identifying the peripheral subgroup in its centralizer. The latter graph of groups is $\mathcal{G}\left(\mathrm{Comp}_{i+1}\right)$.

2B. Assume that $\eta(P)$ can be conjugated to the centralizer $C$ (in $C o m p_{i}$ ) either of $\eta\left(G_{e}\right)$ for some edge $e$ in $\mathcal{G}_{i}$ that connects two rigid vertex groups or of the image of the peripheral group of some abelian vertex group (i.e., a vertex group whose vertex belongs to $\left.V_{A}\right)$ in $\mathcal{G}_{i}$. Then $C o m p_{i+1}$ is the group corresponding to the graph of groups obtained by gluing to $\mathcal{G}\left(C o m p_{i}\right)$ a free abelian flat of rank $n$ along $C$.

Case 3. Suppose $e_{i+1}:=(u, v)$ connects a surface type vertex with a rigid vertex (i.e., a vertex that belongs to $V_{R}$ ). We further take cases according to whether the surface type vertex belongs to $\mathcal{G}_{i}$ or not:

3A. Assume that the surface vertex group $G_{v}$ does not belong to $\mathcal{G}_{i}$. Then $C_{\text {omp }} p_{i+1}$ is the amalgamated free product $\operatorname{Comp}_{i} *_{G_{e_{i+1}}} \tilde{G}_{v}$ where $\tilde{G}_{v}$ is an isomorphic copy of $G_{v}$ witnessed by the isomorphism $f: G_{v} \rightarrow \tilde{G}_{v}$, and the edge group embeddings $\tilde{f}_{e_{i+1}}, \tilde{f}_{\bar{e}_{i+1}}$ are defined as follows: $\tilde{f}_{e_{i+1}}=f \circ f_{e_{i+1}}$ and $\tilde{f}_{\bar{e}_{i+1}}=\eta \circ f_{\bar{e}_{i+1}}$, where $f_{e_{i}+1}, f_{\bar{e}_{i+1}}$ are the injective morphisms that correspond to the edge group of the splitting $\pi_{1}\left(\mathcal{G}_{i}\right) *_{G_{e_{i+1}}} G_{v}$.

3B. Assume that the surface vertex group $G_{u}$ belongs to $\mathcal{G}_{i}$. Then by our recursive hypothesis there exists an isomorphic copy of $G_{u}$, say $f: G_{u} \rightarrow$ $\tilde{G}_{u}$, in $C_{o m p}$. We define $\operatorname{Comp}_{i+1}$ to be the HNN extension $\operatorname{Comp}_{i} *_{G_{e_{i+1}}}$, where the edge group embeddings $\tilde{f}_{e_{i+1}}, \tilde{f}_{\bar{e}_{i+1}}$ are defined as follows: $\tilde{f}_{e_{i+1}}=$ $\eta \circ f_{e_{i+1}}$ and $\tilde{f}_{\bar{e}_{i+1}}=f \circ f_{\bar{e}_{i+1}}$, where $f_{e_{i+1}}, f_{\bar{e}_{i+1}}$ are the injective morphisms that correspond to the edge group of the splitting $\pi_{1}\left(\mathcal{G}_{i}\right) *_{G_{e_{i+1}}} G_{v}$ (in the case $v$ is not in $\mathcal{G}_{i}$ ) or of the splitting $\pi_{1}\left(\mathcal{G}_{i}\right) *_{G_{e_{i+1}}}$ (in the case $v$ is in $\mathcal{G}_{i}$ ).

Finally the group $\operatorname{Comp}(\mathcal{G}(G), \eta):=\operatorname{Comp}_{m}$ is called the completion of $G$ with respect to $\mathcal{G}(G)$, the sequence $\mathcal{G}_{0} \subset \cdots \subset \mathcal{G}_{m}$, and $\eta$.

The completion of a group $G$ with respect to a strict map $\eta: G \rightarrow L$ has a natural structure of a floor over $L$. Moreover, Sela has proved [16, Lemma 1.13] that $G$ admits a natural embedding into its completion. 
Lemma 5.5. Let $G$ be a group and let $\left(\mathcal{G}(G),\left(V_{S}, V_{A}, V_{R}\right)\right)$ be a GAD for $G$. Let $L$ be a limit group and let $\eta: G \rightarrow L$ be such that $(\mathcal{G}(G), \eta)$ is strict. Let $\operatorname{Comp}(\mathcal{G}(G), \eta)$ be the completion of $G$ with respect to $\mathcal{G}(G)$ and $\eta$. Then $G$ admits a natural embedding to $\operatorname{Comp}(\mathcal{G}(G), \eta)$.

Proof. Let $\mathcal{G}_{0} \subset \mathcal{G}_{1} \subset \cdots \subset \mathcal{G}_{m}:=\mathcal{G}(G)$ be the sequence of subgraphs of groups that covers the graph of groups $\mathcal{G}(G)$ and with respect to which we have constructed the completion $\operatorname{Comp}(\mathcal{G}(G), \eta)$. We will prove by induction that for each $i \leq m$, there exists an injective map $f_{i}: \pi_{1}\left(\mathcal{G}_{i}\right) \rightarrow C o m p_{i}$ such that $f_{i+1} \supset f_{i}$ and $\bigcup f_{i}:=$ $f: G \rightarrow \operatorname{Comp}(\mathcal{G}(G), \eta)$ agrees with $\eta$ up to conjugation, by an element that is either trivial or does not live in $L$, in the vertex groups whose vertices belong to $V_{R}$ in the GAD for $G$.

Base step. We take $f_{0}$ to be $\eta \uparrow \pi_{1}\left(\mathcal{G}_{0}\right)$. Since we have assumed that the unique vertex in $\mathcal{G}_{0}$ belongs $V_{R}$ and $C_{0 m p}$ is $L$, one sees that $f_{0}: \pi_{1}\left(\mathcal{G}_{0}\right) \rightarrow C o m p_{0}$ is injective and respects our hypothesis on vertex groups whose vertices belong to $V_{R}$.

Inductive step. Let $f_{i}: \pi_{1}\left(\mathcal{G}_{i}\right) \rightarrow$ Comp $_{i}$ be the morphism that satisfies our induction hypothesis. We find an injective morphism $f_{i+1}: \pi_{1}\left(\mathcal{G}_{i+1}\right) \rightarrow C_{o m p} p_{i+1}$ that extends $f_{i}$ and satisfies the hypothesis on vertex groups whose vertices belong to $V_{R}$. We take cases according to the initial and terminal vertices of the edge $e:=(u, v) \in \mathcal{G}_{i+1} \backslash \mathcal{G}_{i}$.

- Suppose we are in Case 1A of Definition 5.4 Then $\pi_{1}\left(\mathcal{G}_{i+1}\right)$ is either the amalgamated free product $\pi_{1}\left(\mathcal{G}_{i}\right) *_{G_{e}} G_{v}$ (if $v \notin \mathcal{G}_{i}$ ) or the HNN extension $\pi_{1}\left(\mathcal{G}_{i}\right) *_{G_{e}}$ (if $v \in \mathcal{G}_{i}$ ) and $C o m p_{i+1}$ is the amalgamated free product $C o m p_{i} *_{C}(C \oplus\langle z\rangle)$ where $C$ is the centralizer of $\eta\left(G_{e}\right)$ in $C o m p_{i}$.

Suppose that $\pi_{1}\left(\mathcal{G}_{i+1}\right)$ is an amalgamated free product. By the induction hypothesis $f_{i} \uparrow G_{u}=\operatorname{Conj}\left(\gamma_{u}\right) \circ \eta \uparrow G_{u}$. We define $f_{i+1}$ to agree with $f_{i}$ on $\pi_{1}\left(\mathcal{G}_{i}\right)$ and $f_{i+1}(g)=\gamma_{u} z \eta(g) z^{-1} \gamma_{u}^{-1}$ for $g \in G_{v}$. Note that obviously $\gamma_{u} z$ does not belong to $C o m p_{i}$; thus it does not belong to $L$. The map $f_{i+1}$ is indeed a morphism since for any $g \in G_{e}$ we have that $f_{\bar{e}}(g)$ is an element that lives in $G_{u}$; thus $f_{i+1}\left(f_{\bar{e}}(g)\right)=f_{i}\left(f_{\bar{e}}(g)\right)=$ $\gamma_{u} \eta\left(f_{\bar{e}}(g)\right) \gamma_{u}^{-1}$. On the other hand $f_{i+1}\left(f_{e}(g)\right)=\gamma_{u} z \eta\left(f_{e}(g)\right) z^{-1} \gamma_{u}^{-1}$, and since $\eta\left(f_{e}(g)\right)$ is in $C$ we get that $f_{i+1}\left(f_{e}(g)\right)=\gamma_{u} \eta\left(f_{e}(g)\right) \gamma_{u}^{-1}$. Therefore, since $\eta\left(f_{\bar{e}}(g)\right)=\eta\left(f_{e}(g)\right)$, we see that $f_{i+1}\left(f_{e}(g)\right)=f_{i+1}\left(f_{\bar{e}}(g)\right)$. We continue by proving that $f_{i+1}$ is injective. Let $g:=a_{1} b_{1} \cdots a_{n} b_{n}$ be an element of $\pi_{1}\left(\mathcal{G}_{i}\right) *_{G_{e}} G_{v}$ in reduced form. Then $f_{i+1}(g)=$ $f_{i}\left(a_{1}\right) \gamma_{u} z \eta\left(b_{1}\right) z^{-1} \gamma_{u}^{-1} \cdots f_{i}\left(a_{n}\right) \gamma_{u} z \eta\left(b_{n}\right) z^{-1} \gamma_{u}^{-1}$. We show that this form is reduced with respect to $\operatorname{Comp}_{i} *_{C}(C \oplus\langle z\rangle)$. Suppose not. Then either $\gamma_{u}^{-1} f_{i}\left(a_{j}\right) \gamma_{u}$, for some $2 \leq j \leq n$, or $\eta\left(b_{j}\right)$ for some $j \leq n$ is in $C$. In the first case this means that $\gamma_{u}^{-1} f_{i}\left(a_{j}\right) \gamma_{u}$ commutes with some (any) nontrivial element, say $\gamma$, of $\eta\left(G_{e}\right)$. Thus $f_{i}\left(a_{j}\right)$ commutes with $\gamma_{u} \gamma \gamma_{u}^{-1}$, but $\gamma_{u} \gamma \gamma_{u}^{-1}$ is the image of an element in $G_{e}$ under $f_{i}$, and since $f_{i}$ is injective we have that $a_{j}$ commutes with an element of $G_{e}$ by the maximality condition of $G_{e}$. This shows that $a_{j}$ belongs to $G_{e}$, a contradiction. In the second case, this means that $\eta\left(b_{j}\right)$ commutes with some (any) nontrivial element, say $\gamma$, of $\eta\left(G_{e}\right)$. Thus since $\eta$ is injective on $G_{v}$, we see that $b_{j}$ commutes with an element of $G_{e}$. By the maximality condition of $G_{e}, b_{j}$ must belong to $G_{e}$, a contradiction. 
Suppose that $\pi_{1}\left(\mathcal{G}_{i+1}\right)$ is an HNN extension. By the induction hypothesis $f_{i} \uparrow G_{u}=\operatorname{Conj}\left(\gamma_{u}\right) \circ \eta \uparrow G_{u}, f_{i} \uparrow G_{v}=\operatorname{Conj}\left(\gamma_{v}\right) \circ \eta \uparrow G_{v}$. We define $f_{i+1}$ to agree with $f_{i}$ on $\pi_{1}\left(\mathcal{G}_{i}\right)$ and $f_{i+1}(t)=\gamma_{v} \eta(t) z \gamma_{u}^{-1}$, where $t$ is the stable letter of the HNN extension. The map $f_{i+1}$ is indeed a morphism, since for any $g \in G_{e}$ we have that $f_{e}(g)$ is an element that lives in $G_{v}$; thus $f_{i+1}\left(f_{e}(g)\right)=f_{i}\left(f_{e}(g)\right)=\gamma_{v} \eta\left(f_{e}(g)\right) \gamma_{v}^{-1}$. On the other hand $f_{\bar{e}}(g)$ is an element that lives in $G_{u}$. Thus $f_{i+1}\left(t f_{\bar{e}}(g) t^{-1}\right)=$ $\gamma_{v} \eta(t) z \gamma_{u}^{-1} \gamma_{u} \eta\left(f_{\bar{e}}(g)\right) \gamma_{u}^{-1} \gamma_{u} z^{-1} \eta(t)^{-1} \gamma_{v}^{-1}=\gamma_{v} \eta\left(t f_{\bar{e}}(g) t^{-1}\right) \gamma_{v}^{-1}$, and it follows that $f_{i+1}$ is a morphism. We continue by proving that $f_{i+1}$ is injective. Let $g:=g_{0} t^{\epsilon_{1}} g_{1} t^{\epsilon_{2}} \cdots t^{\epsilon_{n}} g_{n}$ with $\epsilon_{i} \in\{-1,1\}$ be an element in reduced form with respect to the HNN extension. Then $f_{i+1}(g)=$ $f_{i}\left(g_{0}\right)\left(\gamma_{v} \eta(t) z \gamma_{u}^{-1}\right)^{\epsilon_{1}} f_{i}\left(g_{1}\right)\left(\gamma_{v} \eta(t) z \gamma_{u}^{-1}\right)^{\epsilon_{2}} \cdots\left(\gamma_{v} \eta(t) z \gamma_{u}^{-1}\right)^{\epsilon_{n}} f_{i}\left(g_{n}\right)$. We will show by induction that for every $n \geq 1$, if $g$ is an element of $\pi_{1}\left(\mathcal{G}_{i+1}\right)$ of length $n$ (in reduced form) with respect to the HNN extension that $\pi_{1}\left(\mathcal{G}_{i+1}\right)$ admits, then $f_{i+1}(g)$ can be put in reduced form of length at least one with respect to the amalgamated free product $\operatorname{Comp}_{i} *_{C}(C \oplus\langle z\rangle)$ that Comp $_{i+1}$ admits. Moreover $f_{i+1}(g)$ ends with either $z \gamma_{u}^{-1} f_{i}\left(g_{n}\right)$ or $z^{-1} \eta(t)^{-1} \gamma_{v}^{-1} f_{i}\left(g_{n}\right)$ depending on whether $\epsilon_{n}$ is positive or negative. For the base step $(n=1)$, the result is obvious. Suppose it is true for every $k<$ $n$; we show it is true for $n$. We take cases with respect to whether $\epsilon_{n-1}, \epsilon_{n}$ are positive or negative. Since the cases when both are negative or both are positive are symmetric we assume that both are positive and we leave the symmetric case as an exercise. Thus, $f_{i+1}(g)=f_{i+1}\left(g_{0} t^{\epsilon_{1}} g_{1} t^{\epsilon_{2}} \cdots t g_{n-1}\right)$. $f_{i+1}\left(t g_{n}\right)$, and by the induction hypothesis $f_{i+1}\left(g_{0} t^{\epsilon_{1}} g_{1} t^{\epsilon_{2}} \cdots t^{\epsilon_{n-1}} g_{n-1}\right)$ can be put in reduced form of length at least one that ends with $z \gamma_{u}^{-1} f_{i}\left(g_{n-1}\right)$. Therefore $f_{i+1}(g)=\gamma z \gamma_{u}^{-1} f_{i}\left(g_{n-1}\right) \gamma_{v} \eta(t) z \gamma_{u}^{-1} f_{i}\left(g_{n}\right)$. This latter element has the desired properties, since if $\gamma_{u}^{-1} f_{i}\left(g_{n-1}\right) \gamma_{v} \eta(t)$ belongs to $C$, then we consider $z \gamma_{u}^{-1} f_{i}\left(g_{n-1}\right) \gamma_{v} \eta(t) z$ as an element of $C \oplus\langle z\rangle \backslash C$, and if not, then already the element is in reduced form ending with $z \gamma_{u}^{-1} f_{i}\left(g_{n}\right)$. We now treat the case where $\epsilon_{n-1}=1$ and $\epsilon_{n}=-1$. In this case $f_{i+1}(g)=$ $f_{i+1}\left(g_{0} t^{\epsilon_{1}} g_{1} t^{\epsilon_{2}} \cdots t g_{n-1}\right) \cdot f_{i+1}\left(t^{-1} g_{n}\right)$, and by the induction hypothesis

$$
f_{i+1}\left(g_{0} t^{\epsilon_{1}} g_{1} t^{\epsilon_{2}} \ldots t g_{n-1}\right)
$$

can be put in reduced form of length at least one that ends with $z \gamma_{u}^{-1} f_{i}\left(g_{n-1}\right)$. Thus, $f_{i+1}(g)=\gamma z \gamma_{u}^{-1} f_{i}\left(g_{n-1}\right) \gamma_{u} z^{-1} \eta(t)^{-1} \gamma_{v}^{-1} f_{i}\left(g_{n}\right)$. It is enough to show that $\gamma_{u}^{-1} f_{i}\left(g_{n-1}\right) \gamma_{u}$ does not belong to the centralizer $C$ of $\eta\left(G_{e}\right)$. Suppose, for a contradiction, that it does. Then $\gamma_{u}^{-1} f_{i}\left(g_{n-1}\right) \gamma_{u}$ commutes with some (any) element of $\eta\left(G_{e}\right)$; such an element can be written as $\gamma_{u}^{-1} f_{i}(\beta) \gamma_{u}$ for some $\beta \in G_{u}$. Therefore, $f_{i}\left(g_{n-1}\right)$ commutes with $f_{i}(b)$, and since $f_{i}$ is injective, we see that $g_{n-1}$ commutes with $b$. We can now use the maximality of $f_{\bar{e}}\left(G_{e}\right)$ to conclude that $g_{n-1}$ belongs to it, contradicting the reduced form for $g$. The case where $\epsilon_{n-1}=-1$ and $\epsilon_{n}=1$ is symmetric to the previous case, and we leave it to the reader.

- Suppose we are in case 1B of Definition 5.4. Suppose the centralizer of $\eta\left(G_{e}\right)$ (in $C o m p_{i}$ ) can be conjugated by the element $\gamma$ into $C$, where $C$ satisfies the hypothesis of case 1B. Then $\pi_{1}\left(\mathcal{G}_{i+1}\right)$ is either the amalgamated free product $\pi_{1}\left(\mathcal{G}_{i}\right) *_{G_{e}} G_{v}$ (if $v \notin \mathcal{G}_{i}$ ) or the HNN extension $\pi_{1}\left(\mathcal{G}_{i}\right) *_{G_{e}}$ (if $\left.v \in \mathcal{G}_{i}\right)$, and $\operatorname{Comp}_{i+1}$ is the amalgamated free product $\operatorname{Comp}_{i} *_{C}(C \oplus\langle z\rangle)$. 
Suppose that $\pi_{1}\left(\mathcal{G}_{i+1}\right)$ is an amalgamated free product. By the induction hypothesis $f_{i} \uparrow G_{u}=\operatorname{Conj}\left(\gamma_{u}\right) \circ \eta \uparrow G_{u}$. We define $f_{i+1}$ to agree with $f_{i}$ on $\pi_{1}\left(\mathcal{G}_{i}\right)$ and $f_{i+1}(g)=\gamma_{u} \gamma^{-1} z \gamma \eta(g) \gamma^{-1} z^{-1} \gamma \gamma_{u}^{-1}$ for $g \in G_{v}$. It is not hard to check that $f_{i+1}$ is a morphism, the reason being that for any element $g$ of $G_{e}$, since $\gamma \eta\left(f_{e}(g)\right) \gamma^{-1}$ belongs to $C$, it commutes with $z$; thus $f_{i+1}\left(f_{e}(g)\right)=\gamma_{u} \eta\left(f_{e}(g)\right) \gamma_{u}^{-1}$ and this is enough. We next prove that $f_{i+1}$ is injective. Let $g:=a_{1} b_{1} \cdots a_{n} b_{n}$ be an element of $\pi_{1}\left(\mathcal{G}_{i}\right) *_{G_{e}} G_{v}$ in reduced form. Then

$$
f_{i+1}(g)=f_{i}\left(a_{1}\right) \gamma_{u} \gamma^{-1} z \gamma \eta\left(b_{1}\right) \gamma^{-1} z^{-1} \gamma \gamma_{u}^{-1} \cdots f_{i}\left(a_{n}\right) \gamma_{u} \gamma^{-1} z \gamma \eta\left(b_{n}\right) \gamma^{-1} z^{-1} \gamma \gamma_{u}^{-1}
$$

We show that this form is reduced with respect to $C o m p_{i} *_{C}(C \oplus\langle z\rangle)$. Suppose not. Then either $\gamma \gamma_{u}^{-1} f_{i}\left(a_{j}\right) \gamma_{u} \gamma^{-1}$, for some $2 \leq j \leq n$, or $\gamma \eta\left(b_{j}\right) \gamma^{-1}$ for some $j \leq n$ is in $C$. In the first case this means that $\gamma \gamma_{u}^{-1} f_{i}\left(a_{j}\right) \gamma_{u} \gamma^{-1}$ commutes with some (any) nontrivial element, say $\beta$, of $\gamma \eta\left(G_{e}\right) \gamma^{-1}$. Thus $\gamma_{u}^{-1} f_{i}\left(a_{j}\right) \gamma_{u}$ commutes with $\gamma^{-1} \beta \gamma$, which is a nontrivial element of $\eta\left(G_{e}\right)$. Thus $f_{i}\left(a_{j}\right)$ commutes with $\gamma_{u} \gamma^{-1} \beta \gamma \gamma_{u}^{-1}$, and the latter can be taken to be the image of some element of $G_{e}$ under $f_{i}$. The injectivity of $f_{i}$ tells us that $a_{j}$ commutes with a nontrivial element of $G_{e}$, and the maximality of $G_{e}$ gives us that $a_{j}$ belongs to $G_{e}$, a contradiction. The argumentation for the case where $\gamma \eta\left(b_{j}\right) \gamma^{-1}$, for some $j \leq n$, is in $C$ is similar.

Suppose that $\pi_{1}\left(\mathcal{G}_{i+1}\right)$ is an HNN extension. By the induction hypothesis $f_{i} \uparrow G_{u}=\operatorname{Conj}\left(\gamma_{u}\right) \circ \eta \uparrow G_{u}, f_{i} \uparrow G_{v}=\operatorname{Conj}\left(\gamma_{v}\right) \circ \eta \uparrow G_{v}$. We define $f_{i+1}$ to agree with $f_{i}$ on $\pi_{1}\left(\mathcal{G}_{i}\right)$ and $f_{i+1}(t)=\gamma_{v} \eta(t) \gamma^{-1} z \gamma \gamma_{u}^{-1}$, where $t$ is the stable letter of the HNN extension. One can check in a way similar to the corresponding case $1 \mathrm{~A}$ that $f_{i+1}$ is an injective morphism.

- Suppose we are in case $2 \mathrm{~A}$ of Definition [5.4. Then $\pi_{1}\left(\mathcal{G}_{i+1}\right)$ is the amalgamated free product $\pi_{1}\left(\mathcal{G}_{i}\right) *_{G_{e}} G_{v}$, where $G_{v}$ is a free abelian group of rank $n$ and $C o m p_{i+1}$ is the amalgamated free product $C o m p_{i} *_{C} A$ where $C$ is isomorphic to the centralizer of the image of the peripheral subgroup $P\left(G_{v}\right)$ by $\eta$ (which is actually the same as $G_{e}$ ) and $A$ is the free abelian group $C \oplus \mathbb{Z}^{n}$ with the relations identifying the peripheral subgroup as a subgroup of $C$ and as a subgroup of $\mathbb{Z}^{n}$.

By the induction hypothesis $f_{i} \uparrow G_{u}=\operatorname{Conj}\left(\gamma_{u}\right) \circ \eta \uparrow G_{u}$. We define $f_{i+1}$ to agree with $f_{i}$ on $\pi_{1}\left(\mathcal{G}_{i}\right)$ and $f_{i+1}(g)=\gamma_{u} f(g) \gamma_{u}^{-1}$ for $g \in G_{v}$, where $f: G_{v} \rightarrow \mathbb{Z}^{n}$ is an isomorphism between $G_{v}$ and its copy in $C o m p_{i+1}$. It is not hard to check that $f_{i+1}$ is a morphism: Let $g \in G_{e}$. Then $f_{i+1}\left(f_{e}(g)\right)=$ $\gamma_{u} f\left(f_{e}(g)\right) \gamma_{u}^{-1}$ and $f_{i+1}\left(f_{\bar{e}}(g)\right)=\gamma_{u} \eta\left(f_{\bar{e}}(g)\right) \gamma_{u}^{-1}$. Since $f\left(f_{e}(g)\right)=\eta\left(f_{\bar{e}}(g)\right)$ in $C o m p_{i+1}$, we have what we wanted. We next prove that $f_{i+1}$ is injective. Let $g:=a_{1} b_{1} \cdots a_{n} b_{n}$ be an element of $\pi_{1}\left(\mathcal{G}_{i}\right) *_{G_{e}} G_{v}$ in reduced form. Then we prove that $f_{i+1}(g)=f_{i}\left(a_{1}\right) \gamma_{u} f\left(b_{1}\right) \gamma_{u}^{-1} \cdots f_{i}\left(a_{n}\right) \gamma_{u} f\left(b_{n}\right) \gamma_{u}^{-1}$ is in reduced form with respect to $C_{o m p} *_{C} A$. It is trivial to check that $f\left(b_{j}\right)$ does not live in $\tilde{f}_{e}(C)$ (since $b_{j} \in G_{v} \backslash G_{e}$ ); thus we only need to check that $\gamma_{u}^{-1} f_{i}\left(a_{j}\right) \gamma_{u}$ is not in $\tilde{f}_{\bar{e}}(C)$ for $2 \leq j \leq n$. Suppose not. Then $\gamma_{u}^{-1} f_{i}\left(a_{j}\right) \gamma_{u}$ commutes with some (any) element of $\eta\left(f_{\bar{e}}\left(G_{e}\right)\right)$. Therefore, $f_{i}\left(a_{j}\right)$ commutes with $\gamma_{u} \eta\left(f_{\bar{e}}(g)\right) \gamma_{u}^{-1}$, for some $g \in G_{e}$, but that is the image of $g$ under $f_{i}$, and since $f_{i}$ is injective we see that $a_{j}$ commutes with $f_{\bar{e}}(g)$. By the maximality of $f_{\bar{e}}\left(G_{e}\right)$ we have that $a_{j}$ belongs to it, a contradiction. 
- Suppose we are in case 3A of Definition [5.4. Then $\pi_{1}\left(\mathcal{G}_{i+1}\right)$ is the amalgamated free product $\pi_{1}\left(\mathcal{G}_{i}\right) *_{G_{e}} G_{v}$ and $C_{0 m p} p_{i+1}$ is the amalgamated free product $\operatorname{Comp}_{i} *_{G_{e}} \tilde{G}_{v}$, where $\tilde{G}_{v}$ is an isomorphic copy of $G_{v}$ witnessed by the isomorphism $f: G_{v} \rightarrow \tilde{G}_{v}$.

By the induction hypothesis $f_{i} \uparrow G_{u}=\operatorname{Conj}\left(\gamma_{u}\right) \circ \eta \uparrow G_{u}$. We define $f_{i+1}$ to agree with $f_{i}$ on $\pi_{1}\left(\mathcal{G}_{i}\right)$ and $f_{i+1}(g)=\gamma_{u} f(g) \gamma_{u}^{-1}$ for $g \in G_{v}$. It is not hard to check that $f_{i+1}$ is a morphism: Let $g \in G_{e}$. Then $f_{i+1}\left(f_{e}(g)\right)=$ $\gamma_{u} f\left(f_{e}(g)\right) \gamma_{u}^{-1}$ and $f_{i+1}\left(f_{\bar{e}}(g)\right)=\gamma_{u} \eta\left(f_{\bar{e}}(g)\right) \gamma_{u}^{-1}$. Since $\eta\left(f_{\bar{e}}(g)\right)=f\left(f_{e}(g)\right)$ in $C_{o m p} *_{G_{e}} \tilde{G}_{v}$ we have what we wanted. We next prove that $f_{i+1}$ is injective. Let $g:=a_{1} b_{1} \cdots a_{n} b_{n}$ be an element of $\pi_{1}\left(\mathcal{G}_{i}\right) *_{G_{e}} G_{v}$ in reduced form. Then we prove that $f_{i+1}(g)=f_{i}\left(a_{1}\right) \gamma_{u} f\left(b_{1}\right) \gamma_{u}^{-1} \cdots f_{i}\left(a_{n}\right) \gamma_{u} f\left(b_{n}\right) \gamma_{u}^{-1}$ is in reduced form with respect to $C o m p_{i} *_{G_{e}} \tilde{G}_{v}$. We only need to check that $\gamma_{u}^{-1} f_{i}\left(a_{j}\right) \gamma_{u}$ does not live in $\eta\left(G_{e}\right)$ for any $2 \leq j \leq n$. Suppose, for a contradiction, not. Then $f_{i}\left(a_{j}\right)$ is $\gamma_{u} \eta(\gamma) \gamma_{u}^{-1}$, for some $\gamma \in G_{e}$, but the latter is the image of $\gamma$ under $f_{i}$, and since $f_{i}$ is injective we have that $a_{j}$ is $\gamma$, a contradiction.

- Suppose we are in case 3B of Definition 5.4. Then $\pi_{1}\left(\mathcal{G}_{i+1}\right)$ is the amalgamated free product $\pi_{1}\left(\mathcal{G}_{i}\right) *_{G_{e}} G_{v}$ or the HNN extension $\pi_{1}\left(\mathcal{G}_{i}\right) *_{G_{e}}$, and Comp $_{i+1}$ is the HNN extension $\operatorname{Comp}_{i} *_{G_{e}}$.

Suppose that $\pi_{1}\left(\mathcal{G}_{i+1}\right)$ is an amalgamated free product. By the induction hypothesis $f_{i} \uparrow G_{u}=\operatorname{Conj}\left(\gamma_{u}\right) \circ f$, where $f: G_{u} \rightarrow$ $\tilde{G}_{u}$ is an isomorphism, and $\tilde{G}_{u}$ is the isomorphic copy of $G_{u}$ in $C_{u m p}$. We define $f_{i+1}$ to agree with $f_{i}$ on $\pi_{1}\left(\mathcal{G}_{i}\right)$ and $f_{i+1}(g)=\gamma_{u} t^{-1} \eta(g) t \gamma_{u}^{-1}$ for $g \in G_{v}$, where $t$ is the stable letter of the HNN extension $\operatorname{Comp}_{i} *_{G_{e}}$. It is not hard to check that $f_{i+1}$ is a morphism: Let $g \in G_{e}$. Then $f_{i+1}\left(f_{e}(g)\right)=\gamma_{u} t^{-1} \eta\left(f_{e}(g)\right) t \gamma_{u}^{-1}$ and $f_{i+1}\left(f_{\bar{e}}(g)\right)=\gamma_{u} f\left(f_{\bar{e}}(g)\right) \gamma_{u}^{-1}$. Since $\eta\left(f_{e}(g)\right)=t f\left(f_{\bar{e}}(g)\right) t^{-1}$ in $C_{o m p} *^{*} G_{e}$, we have what we wanted. We next prove that $f_{i+1}$ is injective. Let $g:=a_{1} b_{1} \cdots a_{n} b_{n}$ be an element of $\pi_{1}\left(\mathcal{G}_{i}\right) *_{G_{e}} G_{v}$ in reduced form. Then we prove that $f_{i+1}(g)=$ $f_{i}\left(a_{1}\right) \gamma_{u} t^{-1} \eta\left(b_{1}\right) t \gamma_{u}^{-1} \cdots f_{i}\left(a_{n}\right) \gamma_{u} t^{-1} \eta\left(b_{n}\right) t \gamma_{u}^{-1}$ is in reduced form with respect to $\operatorname{Comp}_{i} *_{G_{e}}$. We need to check that $\eta\left(b_{j}\right)$ is not in $\tilde{f}\left(G_{e}\right)$ (which is actually $\eta\left(f_{e}\left(G_{e}\right)\right)$ ) for any $j \leq n$ and $\gamma_{u}^{-1} f_{i}\left(a_{j}\right) \gamma_{u}$ is not in $\tilde{f}_{\bar{e}}\left(G_{e}\right)$ (which is actually $f\left(f_{\bar{e}}\left(G_{e}\right)\right)$ ) for any $2 \leq j \leq n$. But both follow easily by the fact that $b_{j}$ is in $G_{v} \backslash f_{e}\left(G_{e}\right)$ and $a_{j} \in \pi_{1}\left(\mathcal{G}_{i}\right) \backslash f_{\bar{e}}\left(G_{e}\right)$.

Suppose that $\pi_{1}\left(\mathcal{G}_{i+1}\right)$ is an HNN extension. By the induction hypothesis $f_{i} \uparrow G_{u}=\operatorname{Conj}\left(\gamma_{u}\right) \circ f$, where $f: G_{u} \rightarrow \tilde{G}_{u}$ is an isomorphism, and $\tilde{G}_{u}$ is the isomorphic copy of $G_{u}$ in $\operatorname{Comp}_{i}$, and $f_{i}\left\lceil G_{v}=\operatorname{Conj}\left(\gamma_{v}\right) \circ \eta\right.$. We define $f_{i+1}$ to agree with $f_{i}$ on $\pi_{1}\left(\mathcal{G}_{i}\right)$ and $f_{i+1}(t)=\gamma_{v} \tilde{t} \gamma_{u}^{-1}$, where $\tilde{t}$ is the Bass-Serre element of the HNN extension $\operatorname{Comp}_{i} *_{G_{e}}$. It is not hard to check that $f_{i+1}$ is a morphism: Let $g \in G_{e}$. Then $f_{i+1}\left(f_{e}(g)\right)=\gamma_{v} \eta\left(f_{e}(g)\right) \gamma_{v}^{-1}$ and $\left.f_{i+1}\left(t f_{\bar{e}}(g)\right) t^{-1}\right)=\gamma_{v} \tilde{t} f\left(f_{\bar{e}}\right) \tilde{t}^{-1} \gamma_{v}^{-1}$. Since $\eta\left(f_{e}(g)\right)=\tilde{t} f\left(f_{\bar{e}}\right) \tilde{t}^{-1}$ in $C_{\text {omp }}{ }_{i+1}$, we have what we wanted. We next prove that $f_{i+1}$ is injective. Let $g:=g_{0} t^{\epsilon_{1}} g_{1} t^{\epsilon_{2}} \cdots t^{\epsilon_{n}} g_{n}$, with $\epsilon_{j} \in\{1,-1\}$, be an element in reduced form with respect to the HNN extension $\pi_{1}\left(\mathcal{G}_{i}\right)$. Then we prove that $f_{i+1}(g)=f_{i}\left(g_{0}\right)\left(\gamma_{v} \tilde{t} \gamma_{u}^{-1}\right)^{\epsilon_{1}} f_{i}\left(g_{1}\right) \cdots\left(\gamma_{v} \tilde{t} \gamma_{u}^{-1}\right)^{\epsilon_{n}} f_{i}\left(g_{n}\right)$ is in reduced form. We need to check that when $\epsilon_{j}=1$ and $\epsilon_{j+1}=-1$, then $\gamma_{u}^{-1} f_{i}\left(g_{j}\right) \gamma_{u}$ does not live in $\tilde{f}_{\bar{e}}\left(G_{e}\right)$ (which is actually $f\left(f_{\bar{e}}\left(G_{e}\right)\right)$ ) and when $\epsilon_{j}=-1$ and $\epsilon_{j+1}=1$, then $\gamma_{v}^{-1} f_{i}\left(g_{j}\right) \gamma_{v}$ does not live in $\tilde{f}_{e}\left(G_{e}\right)$ (which is actually 
$\left.\eta\left(f_{e}\left(G_{e}\right)\right)\right)$. We show just the former, since the latter case is symmetric. Suppose, for a contradiction, that $\gamma_{u}^{-1} f_{i}\left(g_{j}\right) \gamma_{u}$ is in $f\left(f_{\bar{e}}\left(G_{e}\right)\right)$. Then there exists an element in $\gamma \in f_{\bar{e}}\left(G_{e}\right)$ such that $f_{i}\left(g_{j}\right)=\gamma_{u} f(\gamma) \gamma_{u}^{-1}$, but then $f_{i}\left(g_{j}\right)=f_{i}(\gamma)$, and since $f_{i}$ is injective we see that $g_{j}=\gamma$, a contradiction.

It is not hard to deduce from the construction of the completion that:

Lemma 5.6. Let Sld be a solid group with respect to a finitely generated subgroup $H$. Let $J S J_{H}(S l d)$ be the abelian JSJ decomposition of Sld with respect to $H$ and let Comp $(S l d, I d)$ be the completion of Sld with respect to Id : Sld $\rightarrow$ Sld and the modification $\left.J \hat{S} J_{H}(S l d)\right)$ of its relative JSJ decomposition. Let $\hat{H}$ be the vertex group in $J \hat{S} J_{H}(S l d)$ that contains $H$ and let $i_{t}, i_{b}$ be the natural embeddings from Sld to $\operatorname{Comp}(S l d$, Id $)$. Then $i_{t}(S l d) \cap i_{b}(S l d)=\hat{H}$.

For the notion of the abelian $J S J$ decomposition of a solid limit group we refer the reader to [15, Theorem 9.2].

5.2. Strictly solid morphisms and families. We start by defining the notion of a degenerate map with respect to a tower over a solid limit group.

Definition 5.7. Let $\mathcal{T}(G, S l d)$ be a tower of height $m$ over a limit group $L$,

$$
\left\{G,\left(\mathcal{G}\left(G^{m}, G^{m-1}\right), r_{m}\right),\left(\mathcal{G}\left(G^{m-1}, G^{m-2}\right), r_{m-1}\right), \ldots,\left(\mathcal{G}\left(G^{1}, G^{0}\right), r_{1}\right), L\right\},
$$

and let $s: L \rightarrow \mathbb{F}$ be a morphism. We say that a morphism $h: G \rightarrow \mathbb{F}$ factors through the tower $\mathcal{T}(G, L)$ based on $s$ if $h=s \circ r_{1} \circ \alpha_{1} \circ \cdots \circ r_{m} \circ \alpha_{m}$ where $\alpha_{i} \in \operatorname{Mod}\left(\mathcal{G}\left(G^{i}, G^{i-1}\right)\right)$.

Definition 5.8. Let $\mathcal{T}(G, S l d)$ be a tower of height $m$ over a solid limit group $S l d$,

$$
\left\{G,\left(\mathcal{G}\left(G^{m}, G^{m-1}\right), r_{m}\right),\left(\mathcal{G}\left(G^{m-1}, G^{m-2}\right), r_{m-1}\right), \ldots,\left(\mathcal{G}\left(G^{1}, G^{0}\right), r_{1}\right), S l d\right\},
$$

and let $s: S l d \rightarrow \mathbb{F}$ be a morphism. We say that $s$ is degenerate if for all morphisms $h: G \rightarrow \mathbb{F}$ that factor through the tower $\mathcal{T}(G, S l d)$ based on $s$ and some $i<m$ one of the following holds:

- an edge group of $\left(\mathcal{G}\left(G^{i+1}, G^{i}\right),\left(V_{S}, V_{A}, V_{R}\right)\right)$ is always mapped to the trivial element;

- a vertex group, $G_{u}$, of $\left(\mathcal{G}\left(G^{i+1}, G^{i}\right),\left(V_{S}, V_{A}, V_{R}\right)\right)$ with $u \notin V_{A}$ is always mapped to a cyclic subgroup of $\mathbb{F}$.

As a matter of fact being degenerate is a condition definable by a system of equations.

Lemma 5.9 (Sela). Let $\mathbb{F}:=\mathbb{F}(\bar{a})$ be a nonabelian free group. Let $\mathcal{T}(G, S l d)$ be a graded tower over a solid limit group Sld $:=\langle\bar{x}, \bar{a} \mid \Sigma(\bar{x}, \bar{a})\rangle$. Then there exists a finite system of equations $\Psi(\bar{x}, \bar{a})=1$ such that a morphism $s:$ Sld $\rightarrow \mathbb{F}$ is degenerate with respect to $\mathcal{T}(G, S l d)$ if and only if $\Psi(s(\bar{x}), \bar{a})=1$.

Definition 5.10 (Strictly solid morphism). Let $S l d$ be a solid group with respect to a finitely generated subgroup $H$. Let $J S J_{H}(S l d)$ be the abelian $J S J$ decomposition of Sld with respect to $H$ and let $\operatorname{Comp}(S l d, I d)$ be the completion of Sld with respect to Id : Sld $\rightarrow$ Sld and the modification $\left.J \hat{S} J_{H}(S l d)\right)$ of its relative $J S J$ decomposition. Let $i_{t}, i_{b}$ be the natural embeddings from $\operatorname{Sld}$ to $\operatorname{Comp}(\operatorname{Sld}, \mathrm{Id})$. The morphisms $h_{1}, h_{2}: S l d \rightarrow \mathbb{F}$ are tower equivalent with respect to $\operatorname{Comp}(S l d$, Id) if there is a morphism $H: \operatorname{Comp}(S l d) \rightarrow \mathbb{F}$ such that $H \circ i_{t}=h_{1}$ and $H \circ i_{b}=h_{2}$. 
Moreover, a morphism $h: S l d \rightarrow \mathbb{F}$ is called strictly solid if it is nondegenerate and it is not tower equivalent with respect to $\operatorname{Comp}(S l d, I d)$ to a flexible morphism.

The relation of tower equivalence between two morphisms is an equivalence relation, and we call the set of nondegenerate morphisms that belong to the class of a strictly solid morphism a strictly solid family.

We prove the following:

Lemma 5.11. Let Sld be a solid limit group with respect to a finitely generated subgroup $H$. Let $h_{1}, h_{2}:$ Sld $\rightarrow \mathbb{F}$ be two morphisms. Then $h_{1}, h_{2}$ are tower equivalent with respect to $\operatorname{Comp}(S l d, \mathrm{Id})$ if and only if they agree up to conjugation in the rigid vertices of the relative JSJ decomposition of Sld and they are identical on the vertex group that contains $H$.

Proof. Let $\mathcal{G}_{0} \subseteq \mathcal{G}_{1} \subseteq \cdots \subseteq \mathcal{G}_{m}:=J S J(S l d)$ be the sequence of the subgraphs of groups used in the construction of the completion $\operatorname{Comp}(S l d, \mathrm{Id})$. We recall that $\mathcal{G}_{0}$ is the vertex group that contains $H$ and it is rigid by definition. Let $f_{b}: S l d \rightarrow$ $\operatorname{Comp}(S l d)$ be the identity map that sends Sld onto the Comp $p_{0}$, and let, for each $i \leq m, g_{i}: \pi_{1}\left(\mathcal{G}_{i}\right) \rightarrow C o m p_{i}$ be the natural injective map as in the proof of Lemma 5.5. We will define recursively a sequence of morphisms $\left(F_{i}\right)_{i \leq m}: C o m p_{i} \rightarrow \mathbb{F}$, so that for each $i \leq m$ the morphism $F_{i}$ :

(1) extends the map $F_{i-1}$;

(2) satisfies $F_{i} \circ f_{b}=h_{1}$; and

(3) satisfies $F_{i} \circ g_{i}=h_{2}\left\lceil\pi_{1}\left(\mathcal{G}_{i}\right)\right.$.

Base step. We define $F_{0}: C o m p_{0} \rightarrow \mathbb{F}$ to be essentially the map $h_{1}$, i.e., a morphism such that $F_{0} \circ f_{b}=h_{1}$. Clearly, since $g_{0}\left(\pi_{1}\left(\mathcal{G}_{0}\right)\right)$ belongs to $C o m p_{0}$ and $h_{1}, h_{2}$ agree on $\pi_{1}\left(\mathcal{G}_{0}\right)$, the morphism $F_{0}$ satisfies the required properties.

Before moving to the recursive step we note that property (2) is automatically satisfied for $i>1$ once property (1) is satisfied.

Recursive step. Suppose there exists a morphism $F_{i}$ that satisfies properties (1)-(3). We define $F_{i+1}$ according to the cases for the edge $e:=(u, v) \in \mathcal{G}_{i+1} \backslash \mathcal{G}_{i}$.

- Suppose we are in case $1 \mathrm{~A}$ of Definition 5.4. Then $\pi_{1}\left(\mathcal{G}_{i+1}\right)$ is either the amalgamated free product $\pi_{1}\left(\mathcal{G}_{i}\right) *_{G_{e}} G_{v}$ (if $v \notin \mathcal{G}_{i}$ ) or the HNN extension $\pi_{1}\left(\mathcal{G}_{i}\right) *_{G_{e}}$ (if $v \in \mathcal{G}_{i}$ ), and $C o m p_{i+1}$ is the amalgamated free product $C o m p_{i} *_{C}(C \oplus\langle z\rangle)$ where $C$ is the centralizer of $G_{e}$ in $C o m p_{i}$. By the hypothesis we have that $h_{1}(g)=r_{v} h_{2}(g) r_{v}^{-1}$ for every $g \in G_{v}$ and $h_{1}(g)=r_{u} h_{2}(g) r_{u}^{-1}$ for every $g \in G_{u}$.

Suppose that $\pi_{1}\left(\mathcal{G}_{i+1}\right)$ is an amalgamated free product. The fact that $h_{1}$ and $h_{2}$ are morphisms induces a relation between $r_{v}$ and $r_{u}$. For every $g \in G_{e}$ we have that $h_{2}\left(f_{\bar{e}}(g)\right)=h_{2}\left(f_{e}(g)\right)$; thus $r_{u}^{-1} h_{1}\left(f_{\bar{e}}(g)\right) r_{u}=$ $r_{v}^{-1} h_{1}\left(f_{e}(g)\right) r_{v}$. The last equation implies that $r_{v} r_{u}^{-1}$ commutes with $h_{1}\left(f_{e}(g)\right)$; thus $r_{v}=c r_{u}$ for some element $c$ in the centralizer of $h_{1}\left(f_{e}(g)\right)$.

By the inductive hypothesis and the definition of the map $g_{i}: \pi_{1}\left(\mathcal{G}_{i}\right) \rightarrow$ $C_{\text {omp }}$ we see that, for every element $g \in G_{u}, F_{i} \circ g_{i}(g)=F_{i}\left(\gamma_{u} f_{b}(g) \gamma_{u}^{-1}\right)=$ $h_{2}(g)=r_{u}^{-1} h_{1}(g) r_{u}$ for some $\gamma_{u} \in C_{C o m p}$. This implies that $r_{u} F_{i}\left(\gamma_{u}\right)$ commutes with $h_{1}(g)$ for every $g \in G_{u}$. Therefore $r_{u} F_{i}\left(\gamma_{u}\right)=d$ for some element $d$ in the intersection of all centralizers $C\left(h_{1}(g)\right)$ for $g \in G_{u}$.

Finally, we define $F_{i+1}$ to agree with $F_{i}$ in $\operatorname{Comp}_{i}$ and $F_{i+1}(z)=$ $d^{-1} r_{u} r_{v}^{-1}$. This definition indeed makes $F_{i+1}$ a morphism as $r_{u} r_{v}^{-1}$ and 
$d$ both commute with the image of $C$ under $F_{i}$. Moreover,

$$
F_{i+1} \circ g_{i+1}(g)=F_{i+1}\left(\gamma_{u} z f_{b}(g) z^{-1} \gamma_{u}^{-1}\right)=r_{v}^{-1} h_{1}(g) r_{v}=h_{2}(g)
$$

for every $g \in G_{v}$, as we wanted.

Suppose that $\pi_{1}\left(\mathcal{G}_{i+1}\right)$ is an HNN extension. By the fact that $h_{1}$ and $h_{2}$ are morphisms we get, for every $g \in G_{e}$, that $h_{1}(t) h_{1}\left(f_{\bar{e}}(g)\right) h_{1}(t)^{-1}=$ $h_{1}\left(f_{e}(g)\right)$ and $h_{2}(t) h_{2}\left(f_{\bar{e}}(g)\right) h_{2}(t)^{-1}=h_{2}\left(f_{e}(g)\right)$.

By the inductive hypothesis and the definition of the map $g_{i}: \pi_{1}\left(\mathcal{G}_{i}\right) \rightarrow$ $C_{\text {Comp }}$ we see that for every element $g \in G_{u}, F_{i} \circ g_{i}(g)=F_{i}\left(\gamma_{u} f_{b}(g) \gamma_{u}^{-1}\right)=$ $h_{2}(g)$. The same line of thought as above gives us that $F_{i} \circ g_{i}(g)=$ $F_{i}\left(\gamma_{v} f_{b}(g) \gamma_{v}^{-1}\right)=h_{2}(g)$ for $g \in G_{v}$.

Finally, we define $F_{i+1}$ to agree with $F_{i}$ in $\operatorname{Comp}_{i}$ and $F_{i+1}(z)=$ $h_{1}(t)^{-1} F_{i}\left(\gamma_{v}\right)^{-1} h_{2}(t) F_{i}\left(\gamma_{u}\right)$. We can easily check that

$$
F_{i+1} \circ g_{i+1}(t)=F_{i+1}\left(\gamma_{v} f_{b}(t) z \gamma_{u}^{-1}\right)=h_{2}(t)
$$

as well as that $F_{i+1}$ is a morphism. For the latter we only need to check that $F_{i+1}(z)$ commutes with $F_{i+1}\left(f_{\bar{e}}(g)\right)$ for some nontrivial $g \in G_{e}$. Indeed,

$$
\begin{aligned}
& F_{i+1}(z)^{-1} F_{i+1}\left(f_{\bar{e}}(g)\right) F_{i+1}(z) \\
& \quad=F_{i}\left(\gamma_{u}\right)^{-1} h_{2}(t)^{-1} F_{i}\left(\gamma_{v}\right) h_{1}(t) F_{i+1}\left(f_{\bar{e}}(g)\right) h_{1}(t)^{-1} F_{i}\left(\gamma_{v}\right)^{-1} h_{2}(t) F_{i}\left(\gamma_{u}\right) .
\end{aligned}
$$

We can replace $h_{1}(t) F_{i+1}\left(f_{\bar{e}}(g)\right) h_{1}(t)^{-1}$ with $F_{i+1}\left(f_{e}(g)\right)$, so

$$
\begin{aligned}
& F_{i+1}(z)^{-1} F_{i+1}\left(f_{\bar{e}}(g)\right) F_{i+1}(z) \\
& \quad=F_{i}\left(\gamma_{u}\right)^{-1} h_{2}(t)^{-1} F_{i}\left(\gamma_{v}\right) F_{i+1}\left(f_{e}(g)\right) F_{i}\left(\gamma_{v}\right)^{-1} h_{2}(t) F_{i}\left(\gamma_{u}\right) .
\end{aligned}
$$

Again we can replace, by the induction hypothesis,

$$
F_{i}\left(\gamma_{v}\right) F_{i+1}\left(f_{e}(g)\right) F_{i}\left(\gamma_{v}\right)^{-1}
$$

with $h_{2}\left(f_{e}(g)\right)$, so

$$
F_{i+1}(z)^{-1} F_{i+1}\left(f_{\bar{e}}(g)\right) F_{i+1}(z)=F_{i}\left(\gamma_{u}\right)^{-1} h_{2}(t)^{-1} h_{2}(g) h_{2}(t) F_{i}\left(\gamma_{u}\right) .
$$

We can continue by replacing $h_{2}(t)^{-1} h_{2}\left(f_{e}(g)\right) h_{2}(t)$ with $F_{i} \circ g_{i}\left(f_{\bar{e}}(g)\right)$, so $F_{i+1}(z)^{-1} F_{i+1}\left(f_{\bar{e}}(g)\right) F_{i+1}(z)=F_{i}\left(\gamma_{u}\right)^{-1} F_{i} \circ g_{i}\left(f_{\bar{e}}(g)\right) F_{i}\left(\gamma_{u}\right)$. And finally, $F_{i}\left(\gamma_{u}\right)^{-1} F_{i} \circ g_{i}\left(f_{\bar{e}}(g)\right) F_{i}\left(\gamma_{u}\right)=F_{i}\left(f_{\bar{e}}(g)\right)$, as we wanted.

- Suppose we are in case 2A of Definition 5.4. Then $\pi_{1}\left(\mathcal{G}_{i+1}\right)$ is the amalgamated free product $\pi_{1}\left(\mathcal{G}_{i}\right) *_{G_{e}} G_{v}$, where $G_{v}$ is a free abelian group of rank $n$ and $C o m p_{i+1}$ is the amalgamated free product $C o m p_{i} *_{C} A$, where $C$ is isomorphic to the centralizer (in $C o m p_{i}$ ) of the peripheral subgroup $P\left(G_{v}\right)$ of $G_{v}$ and $A$ is the free abelian group $C \oplus \mathbb{Z}^{n}$, where $\mathbb{Z}^{n}$ is an isomorphic copy of $G_{v}$ witnessed by $\phi: G_{v} \rightarrow \mathbb{Z}^{n}$, together with the relations identifying the peripheral subgroup as a subgroup of $C$ and as a subgroup of $\mathbb{Z}^{n}$. Moreover, $g_{i+1}(g)=\gamma_{u} \phi(g) \gamma_{u}^{-1}$ for every $g \in G_{u}$, where $G_{u}$ is by our modification the peripheral subgroup of $G_{v}$.

By the hypothesis we have that $h_{1}(g)=r_{u} h_{2}(g) r_{u}^{-1}$ for every $g \in G_{u}$. By the inductive hypothesis we know that $F_{i} \circ g_{i}(g)=F_{i}\left(\gamma_{u} f_{b}(g) \gamma_{u}^{-1}\right)=h_{2}(g)$ for every $g \in G_{u}$, so we have that $F_{i}\left(\gamma_{u}\right) h_{1}(g) F_{i}\left(\gamma_{u}\right)^{-1}=r_{u}^{-1} h_{1}(g) r_{u}(*)$. We define $F_{i+1}$ to agree with $F_{i}$ in $C o m p_{i}$ and

$$
F_{i+1}(\phi(g))=F_{i}\left(\gamma_{u}\right)^{-1} h_{2}(g) F_{i}\left(\gamma_{u}\right)
$$


for every $g \in G_{v}$. It is immediate to check that $F_{i+1} \circ g_{i+1}(g)=h_{2}(g)$ for every $g \in G_{v}$; thus we only check that $F_{i+1}$ is a morphism. Let $g \in G_{u}$. We show that $F_{i}(g)=F_{i+1}(\phi(g))$, as these are the relations in the group $A$. Indeed, $F_{i}(g)=h_{1}(g)$ and $F_{i+1}(\phi(g))=F_{i}\left(\gamma_{u}\right)^{-1} h_{2}(g) F_{i}\left(\gamma_{u}\right)$, and replacing $h_{2}(g)$ by $r_{u}^{-1} h_{1}(g) r_{u}$ we get $F_{i+1}(\phi(g))=F_{i}\left(\gamma_{u}\right)^{-1} r_{u}^{-1} h_{1}(g) r_{u} F_{i}\left(\gamma_{u}\right)$, which is by $(*)$ equal to $h_{1}(g)$, as we wanted.

- Suppose we are in case 3A of Definition 5.4 Then $\pi_{1}\left(\mathcal{G}_{i+1}\right)$ is the amalgamated free product $\pi_{1}\left(\mathcal{G}_{i}\right) *_{G_{e}} G_{v}$, where $G_{v}$ is the fundamental group of a surface, and $C o m p_{i+1}$ is the amalgamated free product $\operatorname{Comp}_{i} *_{G_{e}} \tilde{G}_{v}$, where $\tilde{G}_{v}$ is an isomorphic copy of $G_{v}$ witnessed by $\phi: G_{v} \rightarrow \tilde{G}_{v}$, and the edge group embeddings are $\tilde{f}_{e}=\phi \circ f_{e}$ and $\tilde{f}_{\bar{e}}=f_{b} \circ f_{\bar{e}}$. Moreover, $g_{i+1}(g)=\gamma_{u} \phi(g) \gamma_{u}^{-1}$ for every $g \in G_{u}$, where $G_{u}$ is a rigid vertex.

By the hypothesis we have that $h_{1}(g)=r_{u} h_{2}(g) r_{u}^{-1}$ for every $g \in G_{u}$. By the inductive hypothesis we know that $F_{i} \circ g_{i}(g)=F_{i}\left(\gamma_{u} f_{b}(g) \gamma_{u}^{-1}\right)=h_{2}(g)$ for every $g \in G_{u}$, so we have that $F_{i}\left(\gamma_{u}\right) h_{1}(g) F_{i}\left(\gamma_{u}\right)^{-1}=r_{u}^{-1} h_{1}(g) r_{u}(*)$. We define $F_{i+1}$ to agree with $F_{i}$ in $C o m p_{i}$ and

$$
F_{i+1}(\phi(g))=F_{i}\left(\gamma_{u}\right)^{-1} h_{2}(g) F_{i}\left(\gamma_{u}\right)
$$

for every $g \in G_{v}$. It is easy to see that $F_{i+1} \circ g_{i+1}(g)=h_{2}(g)$ for every $g \in G_{v}$; thus we only check that $F_{i+1}$ is a morphism. Indeed, $F_{i+1}\left(\tilde{f}_{\bar{e}}(g)\right)=h_{1}\left(f_{\bar{e}}(g)\right)$ and $F_{i+1}\left(\phi\left(\tilde{f}_{e}(g)\right)\right)=F_{i}\left(\gamma_{u}\right)^{-1} h_{2}\left(f_{e}(g)\right) F_{i}\left(\gamma_{u}\right)$, and replacing $h_{2}\left(f_{e}(g)\right)$ by $h_{2}\left(f_{\bar{e}}(g)\right)$ and then by $r_{u}^{-1} h_{1}\left(f_{\bar{e}}(g)\right) r_{u}$, which is by $(*)$ equal to $h_{1}\left(f_{\bar{e}}(g)\right)$, we get what we wanted.

- Suppose we are in case 3B of Definition 5.4. Then $\pi_{1}\left(\mathcal{G}_{i+1}\right)$ is the amalgamated free product $\pi_{1}\left(\mathcal{G}_{i}\right) *_{G_{e}} G_{v}$ or the HNN extension $\pi_{1}\left(\mathcal{G}_{i}\right) *_{G_{e}}$, and Comp $_{i+1}$ is the HNN extension $\operatorname{Comp}_{i} *_{G_{e}}$. In this case there exists an isomorphic copy $\phi: G_{u} \rightarrow \tilde{G}_{u}$ of the surface group $G_{u}$ in $C o m p_{i}$, and the edge group embeddings are $\tilde{f}_{e}=f_{b} \circ f_{e}, \tilde{f}_{\bar{e}}=\phi \circ f_{\bar{e}}$.

Suppose that $\pi_{1}\left(\mathcal{G}_{i+1}\right)$ is an amalgamated free product. In this case $g_{i}(g)=\gamma_{u} \phi(g) \gamma_{u}^{-1}$ for every $g \in G_{u}$, and $g_{i+1}(g)=\gamma_{u} t^{-1} f_{b}(g) t \gamma_{u}^{-1}$ for every $g \in G_{v}$, where $t$ is the Bass-Serre element of the HNN extension $\operatorname{Comp}_{i} *_{G_{e}}$. By the hypothesis we have that $h_{1}(g)=r_{v} h_{2}(g) r_{v}^{-1}$ for every $g \in G_{v}$.

We define $F_{i+1}$ to agree with $F_{i}$ in $C o m p_{i}$ and $F_{i+1}(t)=r_{v} F_{i+1}\left(\gamma_{u}\right)$. It is easy to see that $F_{i+1} \circ g_{i+1}(g)=h_{2}(g)$ for every $g \in G_{v}$; thus we only need to check that $F_{i+1}$ is a morphism. Indeed, for every $g \in G_{e}, F_{i+1}\left(\tilde{f}_{e}(g)\right)=$ $h_{1}\left(f_{e}(g)\right)$ and $F_{i+1}\left(t \tilde{f}_{\bar{e}}(g) t^{-1}\right)=F_{i+1}(t) F_{i+1}\left(\phi\left(f_{\bar{e}}(g)\right)\right) F_{i+1}(t)^{-1}$. If, in the latter equation, we replace $F_{i+1}(t)$ with $r_{v} F_{i+1}\left(\gamma_{u}\right)$, we get $F_{i+1}\left(t \tilde{f}_{\bar{e}}(g) t^{-1}\right)$ $=r_{v} F_{i+1}\left(\gamma_{u}\right) F_{i+1}\left(\phi\left(f_{\bar{e}}(g)\right)\right) F_{i+1}\left(\gamma_{u}\right)^{-1} r_{v}^{-1}$, and replacing $\gamma_{u} \phi\left(f_{\bar{e}}(g)\right) \gamma_{u}^{-1}$ with $g_{i+1}\left(f_{\bar{e}}(g)\right)$ we get $F_{i+1}\left(t \tilde{f}_{\bar{e}}(g) t^{-1}\right)=r_{v} F_{i+1} \circ g_{i+1}\left(f_{\bar{e}}(g)\right) r_{v}^{-1}$, which is $r_{v} h_{2}\left(f_{\bar{e}}(g)\right) r_{v}^{-1}$, and finally it is $h_{1}\left(f_{\bar{e}}(g)\right)$, as we wanted.

Suppose that $\pi_{1}\left(\mathcal{G}_{i+1}\right)$ is an HNN extension. In this case $g_{i}(g)=$ $\gamma_{u} \phi(g) \gamma_{u}^{-1}$ for every $g \in G_{u}$, and $g_{i}(g)=\gamma_{v} f_{b}(g) \gamma_{v}^{-1}$ for every $g \in G_{v}$. Moreover $g_{i+1}(t)=\gamma_{v} \tilde{t} \gamma_{u}^{-1}$ where $t$ is the Bass-Serre element of the HNN extension $\pi_{1}\left(\mathcal{G}_{i}\right) *_{G_{e}}$, and $\tilde{t}$ is the Bass-Serre element of the HNN extension $\operatorname{Comp}_{i} *_{G_{e}}$. By the hypothesis we have that $h_{1}(g)=r_{v} h_{2}(g) r_{v}^{-1}$ for every $g \in G_{v}$. 
We define $F_{i+1}$ to agree with $F_{i}$ in $C o m p_{i}$ and

$$
F_{i+1}(\tilde{t})=F_{i+1}\left(\gamma_{v}\right)^{-1} h_{2}(t) F_{i+1}\left(\gamma_{u}\right) .
$$

It is easy to see that $F_{i+1} \circ g_{i+1}(t)=h_{2}(t)$; thus we only need to check that $F_{i+1}$ is a morphism. Indeed, $F_{i+1}\left(\tilde{f}_{e}(g)\right)=h_{1}\left(f_{e}(g)\right)$ and $F_{i+1}\left(\tilde{t} \tilde{f}_{\bar{e}}(g) \tilde{t}^{-1}\right)=$ $F_{i+1}\left(\gamma_{v}\right)^{-1} h_{2}(t) F_{i+1}\left(\gamma_{u}\right) F_{i+1}\left(\tilde{f}_{\bar{e}}(g)\right) F_{i+1}\left(\gamma_{u}\right)^{-1} h_{2}(t)^{-1} F_{i+1}\left(\gamma_{v}\right)$. If, in the latter equation, we replace $\gamma_{u} \tilde{f}_{\bar{e}}(g) \gamma_{u}^{-1}$ with $g_{i+1}\left(f_{\bar{e}}(g)\right)$, we get

$$
F_{i+1}\left(\tilde{t} \tilde{f}_{\bar{e}}(g) \tilde{t}^{-1}\right)=F_{i+1}\left(\gamma_{v}\right)^{-1} h_{2}(t) h_{2}\left(f_{\bar{e}}(g)\right) h_{2}(t)^{-1} F_{i+1}\left(\gamma_{v}\right),
$$

and replacing $t f_{\bar{e}}(g) t^{-1}$ with $f_{e}(g)$ we get

$$
F_{i+1}\left(\tilde{t} \tilde{f}_{\bar{e}}(g) \tilde{t}^{-1}\right)=F_{i+1}\left(\gamma_{v}\right)^{-1} h_{2}\left(f_{e}(g)\right) F_{i+1}\left(\gamma_{v}\right)
$$

which in turn is equal to $F_{i+1}\left(\gamma_{v}\right)^{-1} r_{v}^{-1} h_{1}\left(f_{e}(g)\right) r_{v} F_{i+1}\left(\gamma_{v}\right)$, which finally equals $h_{1}\left(f_{e}(g)\right)$, as we wanted.

\section{Test sequences, Diophantine envelopes, And applications}

In this section we record a notion that we will use extensively throughout the rest of the paper: the notion of a test sequence over a tower (see [16, p. 222]). A test sequence is a sequence of morphisms from a group that has the structure of a tower to a free group that, roughly speaking, witnesses the tower structure of the group in the limit action. The corresponding notion in the work of Kharlampovich-Myasnikov [6] is the notion of a generic family. We give more details in subsection 6.1.

As we have already noted in the introduction of our paper, it is hard to decide when a subset of some cartesian product of a nonabelian free group $\mathbb{F}$ is definable in $\mathbb{F}$. Our main idea is that one can deduce certain properties of a definable set through "generic" points in its envelope. The envelope of a definable set consists of a finite set of diophantine sets which moreover have a geometric structure. The union of the diophantine sets that take part in the envelope "covers" the definable set, and in addition "generic" elements (with respect to the geometric structure of each diophantine set) live in the definable set. In subsection 6.3 we give all the details.

In the final subsection we explain the connections between the geometric tools developed and model theory. We prove some variations of Merzlyakov's theorem that make apparent the usefulness of passing from an arbitrary definable set to the diophantine sets in its envelope.

6.1. Test sequences. We begin by giving some examples of groups that have the structure of a tower and we define the notion of a test sequence for them. The simplest cases of groups that admit a structure of a tower are finitely generated free groups and free abelian groups.

For the rest of this section we fix a nonabelian free group $\mathbb{F}$ and a basis of $\mathbb{F}$ with respect to which we will measure the length of elements of $\mathbb{F}$.

Definition 6.1. Let $\left\langle x_{1}, \ldots, x_{k}\right\rangle$ be a free group of rank $k$. Then a sequence of morphisms $\left(h_{n}\right)_{n<\omega}:\langle\bar{x}\rangle \rightarrow \mathbb{F}$ is a test sequence with respect to $\langle\bar{x}\rangle$ if $h_{n}(\bar{x})$ satisfies the small cancellation property $C^{\prime}(1 / n)$ for each $n<\omega$.

Remark 6.2. Without loss of generality we will assume that all the $x_{i}$ 's have similar growth under $\left(h_{n}\right)_{n<\omega}$; i.e., for each $i, j<k$ there are $c_{i, j}, c_{i, j}^{\prime} \in \mathbb{R}^{+}$such that $c_{i, j}<\frac{\left|h_{n}\left(x_{i}\right)\right|_{\mathbb{F}}}{\left|h_{n}\left(x_{j}\right)\right|_{\mathbb{F}}}<c_{i, j}^{\prime}$. 
Definition 6.3. Let $\mathbb{Z}^{k}:=\left\langle x_{1}, \ldots, x_{k} \mid\left[x_{i}, x_{j}\right]\right\rangle$ be a free abelian group of rank $k$. Let $x_{i_{1}}>x_{i_{2}}>\cdots>x_{i_{k}}$ be some order on the $x_{i}$ 's. Then a sequence of morphisms $\left(h_{n}\right)_{n<\omega}: \mathbb{Z}^{k} \rightarrow \mathbb{F}$ is a test sequence with respect to the free abelian group $\mathbb{Z}^{k}$ (and the given order) if $h_{n}\left(x_{i_{1}}\right)=b_{n}^{m_{i_{1}}(n)}, \ldots, h_{n}\left(x_{i_{k}}\right)=b_{n}^{m_{i_{k}}(n)}$, where $b_{n}$ satisfies the small cancellation property $C^{\prime}(1 / n)$ for each $n<\omega$, and $\frac{m_{i_{j+1}}(n)}{m_{i_{j}}(n)}$ goes to 0 as $n \rightarrow \infty$ for every $j<k$.

We continue by defining a test sequence for a tower that consists of a single abelian flat over the parameter free group.

Definition 6.4. Let $\mathbb{Z}^{k}:=\left\langle x_{1}, \ldots, x_{k} \mid\left[x_{i}, x_{j}\right]\right\rangle$ be a free abelian group of rank $k$. Let $G$ be the amalgamated free product $\mathbb{F} *_{C} C \oplus \mathbb{Z}^{k}$, where $C:=\langle c\rangle$ is infinite cyclic and $f_{\bar{e}}(c)=a$ for some $a \in \mathbb{F}$ such that $\langle a\rangle$ is maximal abelian in $\mathbb{F}$ and $f_{e}(c)=c$. Let $x_{i_{1}}>x_{i_{2}}>\cdots>x_{i_{k}}$ be some order on the fixed basis of $\mathbb{Z}^{k}$.

Then a sequence of morphisms $\left(h_{n}\right)_{n<\omega}: G \rightarrow \mathbb{F}$ is a test sequence with respect to (the tower structure of) $G$ (and the given order) if $h_{n} \uparrow \mathbb{F}=$ Id for every $n<\omega$ and $h_{n}\left(x_{i_{1}}\right)=a^{m_{i_{1}}(n)}, \ldots, h_{n}\left(x_{i_{k}}\right)=a^{m_{i_{k}}(n)}$, where $m_{i_{k}} \rightarrow \infty$ and $\frac{m_{i_{j}+1}(n)}{m_{i_{j}}(n)}$ goes to 0 as $n \rightarrow \infty$ for every $j<k$.

Remark 6.5. In particular when in the above definition $k=1$, any infinite sequence $\left(h_{n}\right)_{n<\omega}: G \rightarrow \mathbb{F}$ with $h_{n} \uparrow \mathbb{F}=$ Id is a test sequence with respect to (the tower structure of) $G$.

Definition 6.6. If $\left(h_{n}\right)_{n<\omega}: G \rightarrow H$ is a sequence of morphisms from $G$ to a finitely generated group $H$ (with a fixed generating set) and $g_{1}, g_{2}$ are in $G$, then we say that the growth of $g_{1}$ dominates the growth of $g_{2}\left(\right.$ under $\left.\left(h_{n}\right)_{n<\omega}\right)$ if $\frac{\left|h_{n}\left(g_{2}\right)\right|_{H}}{\left|h_{n}\left(g_{1}\right)\right|_{H}} \rightarrow 0$ as $n \rightarrow \infty$, where $|g|_{H}$ is the word length of $g$ with respect to the fixed generating set for $H$.

When a tower consists only of abelian floors and free products we define a test sequence as follows.

Definition 6.7 (Test sequence for abelian towers). Let $G$ be a group that has the structure of a tower $\mathcal{T}(G, \mathbb{F})$ over $\mathbb{F}$. Suppose $\mathcal{T}(G, \mathbb{F})$ only contains abelian floors and free products. For each abelian floor of the tower we choose an order for the abelian flats or equivalently we assume that each abelian floor consists of a single abelian flat. For each abelian flat $\mathbb{Z}^{k}:=\left\langle x_{1}, \ldots, x_{k} \mid\left[x_{i}, x_{j}\right]\right\rangle$ we choose an order $x_{i_{1}}>x_{i_{2}}>\cdots>x_{i_{k}}$ for the elements of the fixed basis $x_{1}, \ldots, x_{k}$.

Then a sequence of morphisms $\left(h_{n}\right)_{n<\omega}: G \rightarrow \mathbb{F}$ is called a test sequence for $\mathcal{T}(G, \mathbb{F})$ (with respect to the given order of abelian flats and the given order of their generating sets) if the following conditions hold:

- $h_{n} \uparrow \mathbb{F}=$ Id for every $n<\omega$.

- We define the conditions of the restriction of $\left(h_{n}\right)_{n<\omega}$ to the $i+1$-th flat by taking cases according to whether $G^{i+1}$ has a structure of a free product or a free abelian flat over $G^{i}$ :

(1) Suppose $G^{i+1}$ is the free product of $G^{i}$ with $\mathbb{F}_{l}$. Let $\bar{g}=\left(g_{1}, \ldots, g_{n}\right)$ be a generating set for $G_{i}$ and let $\bar{x}=\left(x_{1}, \ldots, x_{l}\right)$ be a basis for $\mathbb{F}_{l}$. Then $h_{n}(\bar{a}, \bar{x})$ satisfies the small cancellation property for each $n$, and the growth of $\bar{x}$ is equivalent to the growth of $\bar{g}$. 
(2) Suppose $G^{i+1}=G^{i} *_{E}\left(E \oplus \mathbb{Z}^{k}\right)$ is obtained from $G^{i}$ by gluing a free abelian flat along $E$ (where $E$ is maximal abelian in $G^{i}$ ). Let $\gamma_{n}$ be the generator of the cyclic group (in $\mathbb{F}$ ) that $E$ is mapped into by $h_{n} \uparrow G^{i}$. Then we define $h_{n}\left(x_{i_{1}}\right)=\gamma_{n}^{m_{i_{1}}(n)}, \ldots, h_{n}\left(x_{i_{k}}\right)=\gamma_{n}^{m_{i_{k}}(n)}$, where $m_{i_{k}}(n) \rightarrow \infty$ and $\frac{m_{i_{j+1}}(n)}{m_{i_{j}}(n)}$ goes to 0 as $n \rightarrow \infty$ for every $j<k$. Moreover the growth of $x_{i_{k}}$ (under $h_{n}$ ) dominates the growth of every element in $G^{i}$ (under $h_{n}$ ).

More generally, in order to define a test sequence for a group $G$ that has the structure of a tower $\mathcal{T}(G, \mathbb{F})$ over $\mathbb{F}$ we first need to order the abelian flats, the surface flats, and the free factors that appear in the floors of the tower, and in addition we need to order the base elements of each abelian flat.

Definition 6.8 (Ordering a tower). Let

$$
\mathcal{T}(G, \mathbb{F}):=\left(\left(\mathcal{G}\left(G^{1}, G^{0}\right), r_{1}\right),\left(\mathcal{G}\left(G^{2}, G^{1}\right), r_{2}\right), \ldots,\left(\mathcal{G}\left(G^{m}, G^{m-1}\right), r_{m}\right)\right)
$$

be a tower of height $m$ over $\mathbb{F}$. Assume that each floor is either a single flat (abelian or surface) or it is a free product. For each $i<m$, let $B_{i}$ be one of the following:

- if $\mathcal{G}\left(G^{i+1}, G^{i}\right)$ is a surface flat that is obtained by gluing a surface $\Sigma_{g, n}$ along its boundary onto some subgroups of $G_{i}$, then $B_{i}$ is the subgroup of $G^{i+1}$ generated by the fundamental group of the surface together with the Bass-Serre elements corresponding to loops generated by the edges of the surface: $\left\langle\pi_{1}\left(\Sigma_{g, n}\right), t_{1}, \ldots, t_{n}\right\rangle$;

- if $\mathcal{G}\left(G^{i+1}, G^{i}\right)$ is an abelian flat obtained from $G^{i}$ by gluing a free abelian group $\mathbb{Z}^{k}$ along the maximal abelian subgroup $E$, then $B_{i}$ is $\mathbb{Z}^{k}$;

- if $\mathcal{G}\left(G^{i+1}, G^{i}\right)$ is the free product $G^{i+1}=G^{i} * \mathbb{F}_{l}$, then $B_{i}$ is $F_{l}$.

We say that $B_{i_{0}}<B_{i_{1}}<\cdots<B_{i_{m}}$ is a legitimate ordering if the following conditions hold:

- $r_{1}\left(B_{i_{0}}\right) \leq \mathbb{F}$;

- for each $0<j<m$, the image of $B_{i_{j}}$ under the retraction $r_{i_{j}+1}$ is a subgroup of the group $\left\langle\mathbb{F}, B_{i_{0}}, \ldots, B_{i_{j}-1}\right\rangle$.

Moreover, the tower $\mathcal{T}(G, \mathbb{F})$ together with a legitimate ordering and an order for the basis of each abelian flat is called an ordered tower. We will denote an ordered tower (for some ordering) by $(\mathcal{T}(G, \mathbb{F}),<$ ).

A tower can always be ordered by choosing an arbitrary order on the surface flats and abelian flats of each floor, then placing the flats of the $i$-th floor before those of the $i+1$-th floor and choosing an order for the basis elements of each abelian flat. On the other hand, one could have more complicated legitimate orderings in the sense that a flat that is part of a higher floor than some other flat can be ordered before this latter flat. In any case, one can obtain from a legitimate ordering a tower structure by reshuffling the floors according to the legitimate order and changing the retractions accordingly. We give some examples.

Example 6.9. We consider the tower over $\mathbb{F}$ with two floors defined as follows:

- The first floor $\mathcal{G}\left(G^{1}, \mathbb{F}\right)$ is a surface flat that is obtained by gluing the surface $\Sigma_{1,1}$ (whose fundamental group is $\left\langle x_{1}, x_{2}\right\rangle$ ) along its boundary onto the subgroup of $\mathbb{F}$ which is generated by the commutator of two noncommuting elements $a_{1}, a_{2}$. Thus, $G^{1}:=\left\langle\mathbb{F}, x_{1}, x_{2} \mid\left[x_{1}, x_{2}\right]=\left[a_{1}, a_{2}\right]\right\rangle$, and $r_{1}: G^{1} \rightarrow \mathbb{F}$ 
is the morphism staying the identity on $\mathbb{F}$ and sending $x_{i}$ to $a_{i}$ for $i \leq 2$. Note that $B_{0}$ is $\left\langle x_{1}, x_{2}\right\rangle$.

- The second floor $\mathcal{G}\left(G^{2}, G^{1}\right)$ is a surface flat that is obtained by gluing the surface $\Sigma_{1,1}$ (whose fundamental group is $\left\langle y_{1}, y_{2}\right\rangle$ ) along its boundary onto the subgroup of $\mathbb{F}$ which is generated by the commutator of two noncommuting elements $b_{1}, b_{2}$. Thus, $G^{2}:=\left\langle G^{1}, y_{1}, y_{2} \mid\left[y_{1}, y_{2}\right]=\left[b_{1}, b_{2}\right]\right\rangle$, and $r_{2}: G^{2} \rightarrow G^{1}$ is the morphism staying the identity on $G^{1}$ and sending $y_{i}$ to $b_{i}$ for $i \leq 2$. Note that $B_{1}$ is $\left\langle y_{1}, y_{2}\right\rangle$.

This tower admits two legitimate orderings: the natural one, $B_{0}<B_{1}$, but also $B_{1}<B_{0}$ since $r_{2}\left(B_{1}\right) \leq \mathbb{F}$ and $r_{1}\left(B_{0}\right) \leq\left\langle\mathbb{F}, B_{1}\right\rangle$.

As noted above one can give $G^{2}$ the following tower structure:

- The first floor $\mathcal{G}\left(\hat{G}^{1}, \mathbb{F}\right)$ is a surface flat that is obtained by gluing the surface $\Sigma_{1,1}$ (whose fundamental group is $\left\langle y_{1}, y_{2}\right\rangle$ ) along its boundary onto the subgroup of $\mathbb{F}$ which is generated by the commutator of $b_{1}, b_{2}$. Thus, $\hat{G}^{1}:=\left\langle\mathbb{F}, y_{1}, y_{2} \mid\left[y_{1}, y_{2}\right]=\left[b_{1}, b_{2}\right]\right\rangle$, and $\hat{r}_{1}: \hat{G}^{1} \rightarrow \mathbb{F}$ is $r_{2}$ restricted on $\left\langle\mathbb{F}, y_{1}, y_{2}\right\rangle$.

- The second floor $\mathcal{G}\left(G^{2}, \hat{G}^{1}\right)$ is a surface flat that is obtained by gluing the surface $\Sigma_{1,1}$ (whose fundamental group is $\left\langle x_{1}, x_{2}\right\rangle$ ) along its boundary onto the subgroup of $\mathbb{F}$ which is generated by the commutator of $a_{1}, a_{2}$. Thus, $G^{2}:=\left\langle\hat{G}^{1}, x_{1}, x_{2} \mid\left[x_{1}, x_{2}\right]=\left[a_{1}, a_{2}\right]\right\rangle$, and $\hat{r}_{2}: G^{2} \rightarrow \hat{G}^{1}$ is the morphism agreeing with $r_{1}$ on $\left\langle\mathbb{F}, x_{1}, x_{2}\right\rangle$ and stays the identity on $\left\langle y_{1}, y_{2}\right\rangle$.

Example 6.10. We consider the tower over $\mathbb{F}$ with two floors defined as follows:

- The first floor $\mathcal{G}\left(G^{1}, \mathbb{F}\right)$ is a surface flat that is obtained by gluing the surface $\Sigma_{1,1}$ (whose fundamental group is $\left\langle x_{1}, x_{2}\right\rangle$ ) along its boundary onto the subgroup of $\mathbb{F}$ which is generated by the commutator of two noncommuting elements $a_{1}, a_{2}$. Thus, $G^{1}:=\left\langle\mathbb{F}, x_{1}, x_{2} \mid\left[x_{1}, x_{2}\right]=\left[a_{1}, a_{2}\right]\right\rangle$, and $r_{1}: G^{1} \rightarrow \mathbb{F}$ is the morphism staying the identity on $\mathbb{F}$ and sending $x_{i}$ to $a_{i}$ for $i \leq 2$. Note that $B_{0}$ is $\left\langle x_{1}, x_{2}\right\rangle$.

- The second floor $\mathcal{G}\left(G^{2}, G^{1}\right)$ is a surface flat that is obtained by gluing the surface $\Sigma_{1,1}$ (whose fundamental group is $\left\langle y_{1}, y_{2}\right\rangle$ ) along its boundary onto the subgroup which is generated by the commutator of $x_{1}$ and $b$ for some nontrivial $b \in \mathbb{F}$. Thus, $G^{2}:=\left\langle G^{1}, y_{1}, y_{2} \mid\left[y_{1}, y_{2}\right]=\left[x_{1}, b\right]\right\rangle$, and $r_{2}: G^{2} \rightarrow G^{1}$ is the morphism staying the identity on $G^{1}$ and sending $y_{1}$ to $x_{1}$ and $y_{2}$ to $b$. Note that $B_{1}$ is $\left\langle y_{1}, y_{2}\right\rangle$.

This tower admits only one legitimate ordering: the natural one, $B_{0}<B_{1}$.

One can easily check that $B_{1}<B_{0}$ is not a legitimate ordering since $r_{2}\left(B_{1}\right)$ is not a subgroup of $\mathbb{F}$.

Remark 6.11. It is not hard to check that:

- a twin tower admits two natural legitimate orderings;

- a tower closure inherits an ordering from the corresponding tower.

Definition 6.12 (Test sequence for a general tower). Suppose $G$ has the structure of a tower $\mathcal{T}(G, \mathbb{F})$ over $\mathbb{F}$ and let $(\mathcal{T}(G, \mathbb{F}),<)$ be some ordering on it. Then a sequence of morphisms $\left(h_{n}\right)_{n<\omega}: G \rightarrow \mathbb{F}$ is a test sequence for this (ordered) tower if it satisfies the combinatorial conditions (i)-(xiv) in [16, p. 222]. 
The existence of a test sequence for a group that has the structure of a tower (for any ordering of the tower) has been proved in [16, Lemma 1.21].

Proposition 6.13. Suppose $G$ has the structure of a tower $\mathcal{T}(G, \mathbb{F})$ over $\mathbb{F}$. Let $(\mathcal{T}(G, \mathbb{F}),<)$ be some ordering. Then a test sequence for $(\mathcal{T}(G, \mathbb{F}),<)$ exists.

The conditions consisting of the definition of a test sequence in [16] are used there to show how a test sequence designs an action of a tower on a limiting real tree. In particular, the following facts (appearing in [16]; cf. Theorem 1.3, Proposition 1.8, Theorem 1.18) about test sequences will be of use for us.

Fact 6.14 (Free product limit action). Let $\mathcal{T}(G, \mathbb{F})$ be a tower and let $\left(h_{n}\right)_{n<\omega}$ : $G \rightarrow \mathbb{F}$ be a test sequence for $\mathcal{T}(G, \mathbb{F})$.

Suppose $G^{i+1}$ is the free product of $G^{i}$ with a group $\mathbb{F}_{l}$ and $\left(h_{n}\left\lceil G^{i+1}\right)_{n<\omega}\right.$ is the restriction of $\left(h_{n}\right)_{n<\omega}$ to $G^{i+1}$. Then, any subsequence of $\left(h_{n}\left\lceil G^{i+1}\right)_{n<\omega}\right.$ that converges, as in Lemma 3.16 induces a faithful action of $G^{i+1}$ on a based real tree $(Y, *)$, with the following properties:

(1) The action $G^{i+1} \curvearrowright Y$ decomposes as a graph of actions in the following way: $\left(G^{i+1} \curvearrowright T,\left\{Y_{u}\right\}_{u \in V(T)},\left\{p_{e}\right\}_{e \in E(T)}\right)$.

(2) The Bass-Serre presentation for $G^{i+1} \curvearrowright T,\left(T_{1}=T_{0}, T_{0}\right)$, is a segment $(u, v)$.

(3) $\operatorname{Stab}_{G}(u):=\mathbb{F}_{l} \curvearrowright Y_{u}$ is a simplicial type action, its Bass-Serre presentation, $\left(Y_{u}^{1}, Y_{u}^{0}, t_{1}, \ldots, t_{l}\right)$ consists of a "star graph" $Y_{u}^{1}:=\left\{\left(x, b_{1}\right), \ldots,\left(x, b_{l}\right)\right\}$ with all of its edges trivially stabilized, a point $Y_{u}^{0}=x$ which is trivially stabilized and Bass-Serre elements $t_{i}=e_{i}$, for $i \leq l$.

(4) $Y_{v}$ is a point and $\operatorname{Stab}_{G}(v)$ is $G^{i}$.

(5) the edge $(u, v)$ is trivially stabilized.

Fact 6.15 (Surface flat limit action). Let $\mathcal{T}(G, \mathbb{F})$ be a tower and let $\left(h_{n}\right)_{n<\omega}$ : $G \rightarrow \mathbb{F}$ be a test sequence with respect to $\mathcal{T}(G, \mathbb{F})$.

Suppose $G^{i+1}$ is a surface flat over $G^{i}:=G_{1}^{i} * \ldots * G_{m}^{i}$, witnessed by $\mathcal{A}\left(G^{i+1}, G^{i}\right)$, and $\left(h_{n}\left\lceil G^{i+1}\right)_{n<\omega}\right.$ is the restriction of $\left(h_{n}\right)_{n<\omega}$ to the $i+1$-flat of $\mathcal{T}(G, \mathbb{F})$.

Then, any subsequence of $\left(h_{n} \uparrow G^{i+1}\right)_{n<\omega}$ that converges, as in Lemma 3.16. induces a faithful action of $G^{i+1}$ on a based real tree $(Y, *)$, with the following properties:

(1) $G^{i+1} \curvearrowright Y$ decomposes as a graph of actions $\left(G^{i+1} \curvearrowright T,\left\{Y_{u}\right\}_{u \in V(T)}\right.$, $\left.\left\{p_{e}\right\}_{e \in E(T)}\right)$, with the action $G^{i+1} \curvearrowright T$ being identical to $\mathcal{A}\left(G^{i+1}, G^{i}\right)$;

(2) if $v$ is not a surface type vertex, then $Y_{v}$ is a point stabilized by the corresponding $G_{j}^{i}$ for some $j \leq m$;

(3) if $u$ is the surface type vertex, then $\operatorname{Stab}_{G}(u)=\pi_{1}\left(\Sigma_{g, l}\right)$ and the action $\operatorname{Stab}_{G}(u) \curvearrowright Y_{u}$ is a surface type action coming from $\pi_{1}\left(\Sigma_{g, l}\right)$.

Fact 6.16 (Abelian flat limit action). Let $\mathcal{T}(G, \mathbb{F})$ be a limit tower and let $\left(h_{n}\right)_{n<\omega}$ : $G \rightarrow \mathbb{F}$ be a test sequence with respect to $\mathcal{T}(G, \mathbb{F})$.

Suppose $G^{i+1}=G^{i} *_{A}(A \oplus \mathbb{Z})$ is obtained from $G^{i}$ by gluing a free abelian flat along $A$ (where $A$ is a maximal abelian subgroup of $\left.G^{i}\right)$ and $\left(h_{n}\left\lceil G^{i+1}\right)_{n<\omega}\right.$ is the restriction of $\left(h_{n}\right)_{n<\omega}$ to the $i+1$-flat of $\mathcal{T}(G, \mathbb{F})$. 
Then any subsequence of $\left(h_{n} \uparrow G^{i+1}\right)_{n<\omega}$ that converges, as in Lemma 3.16. induces a faithful action of $G^{i+1}$ on a based real tree $(Y, *)$, with the following properties:

(1) the action of $G^{i+1}$ on $Y, G^{i+1} \curvearrowright Y$, decomposes as a graph of actions $\left(G^{i+1} \curvearrowright T,\left\{Y_{u}\right\}_{u \in V(T)},\left\{p_{e}\right\}_{e \in E(T)}\right)$;

(2) the Bass-Serre presentation for $G^{i+1} \curvearrowright T,\left(T_{1}=T_{0}, T_{0}\right)$, is a segment $(u, v)$;

(3) $\operatorname{Stab}_{G}(u):=A \oplus \mathbb{Z} \curvearrowright Y_{u}$ is a simplicial type action, and its Bass-Serre presentation, $\left(Y_{u}^{1}, Y_{u}^{0}, t_{e}\right)$, consists of a segment $Y_{u}^{1}:=(a, b)$ whose stabilizer is $A$, a point $Y_{u}^{0}=a$ whose stabilizer is $A$, and a Bass-Serre element $t_{e}$ which is $z$;

(4) $Y_{v}$ is a point, and $\operatorname{Stab}_{G}(v)$ is $G_{i}$;

(5) the edge $(u, v)$ is stabilized by $A$.

6.2. Graded towers and test sequences. We begin by defining the notion of a graded tower.

Definition 6.17. Let $S l d$ be a solid limit group with respect to the finitely generated subgroup $H$ and let $\operatorname{Comp}(S l d):=\operatorname{Comp}\left(J S J_{H}(S l d)\right.$, Id) be its completion with respect to the relative $J S J$ decomposition and the identity map Id : Sld $\rightarrow$ $S l d$. Assume that $J S J_{H}(S l d)$ is not trivial and let $i_{b}, i_{t}: S l d \rightarrow C o m p(S l d)$ be the natural injective maps (where $i_{b}$ is the identity). Let $\mathcal{T}(G, S l d)$ be a tower over Sld, as in Definition 4.8

Then the group corresponding to the graded tower $\mathcal{G} \mathcal{T}(G, S l d)$ is the amalgamated free product $G *_{S l d} \operatorname{Comp}(S l d)$, where $f_{e}: S l d \rightarrow C o m p(S l d)$ is the map $i_{t}$, and $f_{\bar{e}}:$ Sld $\rightarrow G$ maps Sld isomorphically onto the ground floor of $\mathcal{T}(G, S l d)$. Moreover, $\mathcal{G} \mathcal{T}(G, S l d)$ is the tower over $i_{b}(S l d)$ starting with the floors of $\operatorname{Comp}(S l d)$ and continuing with the floors of $\mathcal{T}(G, S l d)$ over the $i_{t}(S l d)$.

Remark 6.18. Concerning Definition 6.17 we note the following:

- In the case where the relative $J S J$ decomposition of $S l d$ is trivial, we take the graded tower to be the tower itself. In the arguments that will follow this will always be a degenerate case, so we will always assume that the relative $J S J$ of $S l d$ is not trivial.

- It is not hard to verify that the natural structure of the graded tower (that is, viewing $G *_{\text {Sld }} \operatorname{Comp}(S l d)$ as a tower over $S l d$ with $G$ as the first floor and gluing the tower over $G$ on top) is a tower structure for $\mathcal{G} \mathcal{T}(G, S l d)$, unless a peg of one of the abelian flats of $\mathcal{T}(G, S l d)$ can be conjugated to a peg of an abelian flat in $\operatorname{Comp}(S l d)$. In such a case, one needs to merge the corresponding flats to have a tower structure.

A graded test sequence for a graded tower $\mathcal{G} \mathcal{T}(G, S l d$ ) (with respect to some ordering) is a sequence of morphisms that restricts to a fixed nondegenerate (with respect to $\mathcal{G} \mathcal{T}(G, S l d)$ ) strictly solid morphism on $i_{b}(S l d)$, and moreover it satisfies the same properties as in Facts 6.14, 6.15, and 6.16 for the floors of $\mathcal{G} \mathcal{T}(G, S l d)$ with the exception that the group acting on the limit action is quotiented by the kernel of the fixed strictly solid morphism.

Fact 6.19. Let $\mathcal{G} \mathcal{T}(G, S l d)$ be a graded tower over $S l d$. Let $s: S l d \rightarrow \mathbb{F}$ be a nondegenerate (with respect to $\mathcal{G} \mathcal{T}(G, S l d)$ ) strictly solid morphism and let 
$\left(h_{n}\right)_{n<\omega}: G *_{\text {Sld }} \operatorname{Comp}(S l d) \rightarrow \mathbb{F}$ be a graded test sequence for $\mathcal{G} \mathcal{T}(G, \mathbb{F})$ (for some ordering) based on $s$. Let $K$ be the kernel of $s$.

Suppose $G^{i+1}$ is the free product of $G^{i}$ with a free group $\mathbb{F}_{l}:=\left\langle e_{1}, \ldots, e_{l}\right\rangle$ and $\left(h_{n}\left\lceil G^{i+1}\right)_{n<\omega}\right.$ is the restriction of $\left(h_{n}\right)_{n<\omega}$ to the $i+1$-flat of $\mathcal{G} \mathcal{T}(G, S l d)$. Then, any subsequence of $\left(h_{n}\left\lceil G^{i+1}\right)_{n<\omega}\right.$ that converges, as in Lemma 3.16, induces a faithful action of $G^{i+1} / K$ on a based real tree $(Y, *)$, with the following properties:

(1) the action $G^{i+1} / K \curvearrowright Y$ decomposes as a graph of actions in the following way: $\left(G^{i+1} / K \curvearrowright T,\left\{Y_{u}\right\}_{u \in V(T)},\left\{p_{e}\right\}_{e \in E(T)}\right)$;

(2) the Bass-Serre presentation for $G^{i+1} / K \curvearrowright T,\left(T_{1}=T_{0}, T_{0}\right)$, is a segment $(u, v)$;

(3) $\operatorname{Stab}_{G}(u):=\mathbb{F}_{l} \curvearrowright Y_{u}$ is a simplicial type action, and its Bass-Serre presentation, $\left(Y_{u}^{1}, Y_{u}^{0}, t_{1}, \ldots, t_{l}\right)$, consists of a "star graph" $Y_{u}^{1}:=$ $\left\{\left(x, b_{1}\right), \ldots,\left(x, b_{l}\right)\right\}$ with all of its edges trivially stabilized, a point $Y_{u}^{0}=x$ which is trivially stabilized, and Bass-Serre elements $t_{i}=e_{i}$ for $i \leq l$;

(4) $Y_{v}$ is a point, and $\operatorname{Stab}_{G}(v)$ is $G^{i} / K$;

(5) the edge $(u, v)$ is trivially stabilized.

Fact 6.20. Let $\mathcal{G} \mathcal{T}(G, S l d)$ be a graded tower over $S l d$. Let $s:$ Sld $\rightarrow \mathbb{F}$ be a nondegenerate (with respect to $\mathcal{G} \mathcal{T}(G, S l d)$ ) strictly solid morphism and let $\left(h_{n}\right)_{n<\omega}: G *_{\text {Sld }} \operatorname{Comp}(S l d) \rightarrow \mathbb{F}$ be a graded test sequence for $\mathcal{G} \mathcal{T}(G, \mathbb{F}$ ) (for some ordering) based on $s$. Let $K$ be the kernel of $s$.

Suppose $G^{i+1}$ is a surface flat over $G^{i}:=G_{1}^{i} * \ldots G_{m}^{i}$, witnessed by $\mathcal{A}\left(G^{i+1}, G^{i}\right)$ and $\left(h_{n}\left\lceil G^{i+1}\right)_{n<\omega}\right.$ is the restriction of $\left(h_{n}\right)_{n<\omega}$ to the $i+1$-flat of $\mathcal{G} \mathcal{T}(G, S l d)$.

Then, any subsequence of $\left(h_{n} \uparrow G^{i+1}\right)_{n<\omega}$ that converges, as in Lemma 3.16 . induces a faithful action of $G^{i+1} / K$ on a based real tree $(Y, *)$, with the following properties:

(1) $G^{i+1} / K \curvearrowright Y$ decomposes as a graph of actions $\left(G^{i+1} / K \curvearrowright T,\left\{Y_{u}\right\}_{u \in V(T)}\right.$, $\left.\left\{p_{e}\right\}_{e \in E(T)}\right)$, with the action $G^{i+1} / K \curvearrowright T$ having the same data as $\mathcal{A}\left(G^{i+1}, G^{i}\right)$, apart from replacing the vertex stabilizer that contains $f_{\bar{e}}(S l d)$ with its quotient $f_{\bar{e}}(S l d) / K$;

(2) if $v$ is not a surface type vertex, then $Y_{v}$ is a point stabilized by the corresponding $G_{j}^{i} / K$ for some $j \leq m$;

(3) if $u$ is the surface type vertex, then $\operatorname{Stab}_{G}(u)=\pi_{1}\left(\Sigma_{g, l}\right)$ and the action $\operatorname{Stab}_{G}(u) \curvearrowright Y_{u}$ is a surface type action coming from $\pi_{1}\left(\Sigma_{g, l}\right)$.

Fact 6.21. Let $\mathcal{G} \mathcal{T}(G, S l d)$ be a graded tower over Sld. Let $s: S l d \rightarrow \mathbb{F}$ be a nondegenerate (with respect to $\mathcal{G} \mathcal{T}(G, S l d)$ ) strictly solid morphism, and let $\left(h_{n}\right)_{n<\omega}: G *_{\text {Sld }} \operatorname{Comp}(S l d) \rightarrow \mathbb{F}$ be a graded test sequence for $\mathcal{G} \mathcal{T}(G, \mathbb{F}$ ) (for some ordering) based on $s$. Let $K$ be the kernel of $s$.

Suppose $G^{i+1}=G^{i} *_{A}(A \oplus \mathbb{Z})$ is obtained from $G^{i}$ by gluing a free abelian flat along $A$ (where $A$ is a maximal abelian subgroup of $\left.G^{i}\right)$ and $\left(h_{n}\left\lceil G^{i+1}\right)_{n<\omega}\right.$ is the restriction of $\left(h_{n}\right)_{n<\omega}$ to the $i+1$-flat of $\mathcal{G} \mathcal{T}(G, S l d)$.

Then any subsequence of $\left(h_{n} \uparrow G^{i+1}\right)_{n<\omega}$ that converges, as in Lemma 3.16. induces a faithful action of $G^{i+1} / K$ on a based real tree $(Y, *)$, with the following properties:

(1) the action of $G^{i+1} / K$ on $Y, G^{i+1} / K \curvearrowright Y$, decomposes as a graph of actions $\left(G^{i+1} / K \curvearrowright T,\left\{Y_{u}\right\}_{u \in V(T)},\left\{p_{e}\right\}_{e \in E(T)}\right)$;

(2) the Bass-Serre presentation for $G^{i+1} / K \curvearrowright T,\left(T_{1}=T_{0}, T_{0}\right)$, is a segment $(u, v)$; 
(3) $\operatorname{Stab}_{G}(u):=A / K \oplus \mathbb{Z} \curvearrowright Y_{u}$ is a simplicial type action, its Bass-Serre presentation, $\left(Y_{u}^{1}, Y_{u}^{0}, t_{e}\right)$, consists of a segment $Y_{u}^{1}:=(a, b)$ whose stabilizer is $A / K$, a point $Y_{u}^{0}=a$ whose stabilizer is $A / K$, and a Bass-Serre element $t_{e}$ which is $z$;

(4) $Y_{v}$ is a point, and $\operatorname{Stab}_{G}(v)$ is $G_{i} / K$;

(5) the edge $(u, v)$ is stabilized by $A / K$.

Using the above properties we can prove the following.

Lemma 6.22. Let Sld be a solid limit group with respect to the finitely generated subgroup $H$. Let $J \hat{S} J_{H}(S l d)$ be the modification of the relative JSJ decomposition of Sld (see Lemma [5.2) and let $\hat{H}$ be the vertex group containing $H$. Let $\operatorname{Comp}(S l d):=\operatorname{Comp}\left(J \hat{S} J_{H}(S l d), \mathrm{Id}\right)$ be its completion with respect to $J \hat{S} J_{H}(S l d)$ and the identity map Id : Sld $\rightarrow$ Sld.

Let $\mathcal{T}(G, S l d)$ be a tower over Sld and let $\left(s_{n}\right)_{n<\omega}:$ Sld $\rightarrow \mathbb{F}$ be a convergent sequence of nondegenerate (with respect to $\mathcal{T}(G, S l d)$ ) strictly solid morphisms with trivial stable kernel.

Let $g \in G$ and assume that for each $n$ there exists a graded test sequence $\left(h_{m}^{n}\right)_{m<\omega}: G *_{\text {Sld }} \operatorname{Comp}(S l d) \rightarrow \mathbb{F}$ based on $s_{n}$ such that $\left|\left\{h_{m}^{n}(g) \mid m<\omega\right\}\right|<\infty$. Then $g \in \hat{H}$.

Proof. The proof is by induction on the height of the floor in the tower $\mathcal{T}(G, S l d)$ that $g$ belongs to. Suppose that $g$ belongs to the ground floor, i.e., $g \in S l d$, and consider the image of $g$ under the edge map of $G *_{S l d} \operatorname{Comp}(S l d)$ in $\operatorname{Comp}(S l d)$. If $g$ belongs to the ground floor of $\operatorname{Comp}(S l d)$, i.e., to $i_{b}(S l d)$, then by Lemma $5.6 \mathrm{~g}$ belongs to $\hat{H}$ as we wanted. On the other hand, if $g$ belongs to $\operatorname{Comp}(S l d)^{i+1} \backslash \operatorname{Comp}(S l d)^{i}$ (in the first floor of the completion) we can choose a morphism $s_{j}: S l d \rightarrow \mathbb{F}$ such that $g \cdot \operatorname{Ker}\left(s_{j}\right)$ belongs to $\operatorname{Comp}(S l d)^{i+1} / \operatorname{Ker}\left(s_{j}\right)$ but not to $C o m p(S l d)^{i} / \operatorname{Ker}\left(s_{j}\right)$. Since the restriction of $\left\{h_{m}^{j}\right\}$ to $\operatorname{Comp}(S l d)$ is a test sequence for $\operatorname{Comp}(S l d)$, by Facts 6.19, 6.20, 6.21 if the set $\left\{h_{m}^{j}(g)\right\}$ is finite, then $g$ stabilizes a point in the limit tree and so belongs to the ground floor of $\operatorname{Comp}(S l d)$, a contradiction.

Similarly, we continue by assuming that $g$ belongs to some higher level $G^{i+1} \backslash G^{i}$ in the tower $\mathcal{T}(G, S l d)$. In this case, we can choose a morphism $s_{j}: S l d \rightarrow \mathbb{F}$ so that $g \cdot \operatorname{Ker}\left(s_{j}\right)$ belongs to $G^{i+1} / \operatorname{Ker}\left(s_{j}\right)$ but not to $G^{i} / \operatorname{Ker}\left(s_{j}\right)$. By the same argument, $g$ must take infinitely many values under $\left(h_{m}^{j}\right)_{m<\omega}$, and this gives a contradiction.

6.3. Diophantine envelopes. In this subsection we start by collecting some theorems of Sela that give an understanding of the "rough" structure of definable sets or parametric families of definable sets in nonabelian free groups. We will use the machinery developed in the previous subsections, namely, towers and test sequences on them.

To give a rough idea before moving to the detailed statements: we would like to have an object (e.g. a definable set equipped with some geometric structure) which we can more easily handle than an "arbitrarily complicated" definable set but as close as possible (in terms of the solution sets) to the definable set. The next theorem as well as Theorem 6.26 can be obtained from Theorem 1.3 of [14] by applying the techniques to construct formal (graded) limit groups and the description of the output of the quantifier elimination for a definable set. 
Theorem 6.23 (Sela - Graded diophantine envelope). Let $\phi(\bar{x}, \bar{y}, \bar{a})$ be a parametric family (with respect to $\bar{y}$ ) of first order formulas over $\mathbb{F}$. Then there exist finitely many, maybe no, graded towers $\left\{\mathcal{G} \mathcal{T}\left(G_{i}, S l d_{i}\right)\right\}_{i \leq k}$, which we call the graded diophantine envelope, where for each $i \leq k, G_{i}$ is generated by $\bar{t}_{i}, \bar{x}, \bar{y}, \bar{a}$, Sld $_{i}:=\left\langle\bar{v}_{i}, \bar{y}, \bar{a}\right\rangle$ is a solid limit group with respect to the subgroup generated by $\langle\bar{y}, \bar{a}\rangle$, for which the following hold:

(i) for each $i \leq k$, there exists a convergent sequence of nondegenerate (with respect to $\left.\mathcal{T}\left(G_{i}, S l d_{i}\right)\right)$ strictly solid morphisms $s_{n}: S l d_{i} \rightarrow \mathbb{F}$ with trivial stable kernel, and for each $n$ there exists a graded test sequence $\left(h_{m}^{n}\right)_{n<\omega}$ : $G_{i} *_{S l d} \operatorname{Comp}\left(\operatorname{Sld}_{i}\right) \rightarrow \mathbb{F}$ based on $s_{n}$, with $\mathbb{F} \models \phi\left(h_{m}^{n}(\bar{x}), h_{m}^{n}(\bar{y}), \bar{a}\right) ;$

(ii) if $\mathbb{F}=\phi\left(\bar{b}_{0}, \bar{c}_{0}, \bar{a}\right)$, then there exist $i \leq k$ and:

(1) a nondegenerate (with respect to $\mathcal{T}\left(G_{i}, S l d_{i}\right)$ ) strictly solid morphism $s: \operatorname{Sld}_{i} \rightarrow \mathbb{F}$ with $s(\bar{y})=\bar{c}_{0}$;

(2) a morphism $h: G_{i} \rightarrow \mathbb{F}$ that extends $s$, factors through the tower $\mathcal{T}\left(G_{i}, S_{l} d_{i}\right)$, and such that $h(\bar{x})=\bar{b}_{0}$;

(3) a graded test sequence $\left(h_{m}\right)_{m<\omega}: G^{i} *_{S l d} \operatorname{Comp}\left(S l d_{i}\right) \rightarrow \mathbb{F}$ based on s such that $\mathbb{F} \models \phi\left(h_{m}(\bar{x}), h_{m}(\bar{y}), \bar{a}\right)$.

We also record the following easy corollary of the above theorem.

Corollary 6.24 (Sela - Diophantine envelope). Let $\phi(\bar{x}, \bar{a})$ be a first order formula over $\mathbb{F}$. Then there exist finitely many, maybe no, towers over $\mathbb{F},\left\{\left(\mathcal{T}\left(G_{i}, \mathbb{F}\right)\right)_{i \leq k}\right\}$, which we call the diophantine envelope, where $G_{i}:=\left\langle\bar{u}_{i}, \bar{x}, \bar{a} \mid \Sigma_{i}\right\rangle$ such that:

(i) $\mathbb{F} \models \phi(\bar{x}, \bar{a}) \rightarrow \exists \bar{u}_{1}, \ldots, \bar{u}_{k}\left(\bigvee_{i=1}^{k} \Sigma_{i}\left(\bar{u}_{i}, \bar{x}, \bar{a}\right)=1\right)$;

(ii) for each $i \leq k$, there exists a test sequence, $\left(h_{n}\right)_{n<\omega}: G_{i} \rightarrow \mathbb{F}$ for $\mathcal{T}\left(G_{i}, \mathbb{F}\right)$, such that $\mathbb{F}=\phi\left(h_{n}(\bar{x}), \bar{a}\right)$.

We use Theorem 6.23 and the definition of a graded test sequence in order to prove the following theorem. Recall that $\exists<\infty x \phi(x)$ reads that the solution set of $\phi(x)$ is nonempty and contains finitely many elements.

Theorem 6.25. Let $\{\phi(\bar{x}, \bar{y}, \bar{a})\}$ be a parametric family (with respect to $\bar{y}$ ) of first order formulas over $\mathbb{F}$. Suppose that $\mathbb{F}=\forall \bar{y} \exists{ }^{<\infty} \bar{x} \phi(\bar{x}, \bar{y}, \bar{a})$. Let $\left\{\left(\mathcal{G} \mathcal{T}\left(G_{i}, S_{l d}\right)\right)_{i \leq k}\right\}$ be a graded envelope for $\phi(\bar{x}, \bar{y}, \bar{a})$.

Let, for each $i \leq k, H_{i}:=\langle\bar{y}, \bar{a}\rangle_{S_{\text {Sld }}}$. Suppose $\hat{H}_{i}$ is the vertex group that contains $H_{i}$ in the modification $J \hat{S} J_{H_{i}}\left(S l d_{i}\right)$ of the relative $J S J$ decomposition of $S l d_{i}$.

Then the tuple of elements $\bar{x}$ of each $G_{i}$ belongs to $\hat{H}_{i}$.

Proof. The first property of the graded envelope (Theorem 6.23)(i)) brings us to the situation of Lemma 6.22 for each graded tower $\mathcal{G T}\left(G_{i}, S l d_{i}\right)$. Thus the result follows.

The following theorem is a consequence of Theorem 1.3 in [14. It has been used in the proof of the stability of the first order theory of nonabelian free groups as well as in showing that this theory is not equational, but also in the (weak) elimination of imaginaries.

Theorem 6.26 (Sela). Let $\mathcal{T}(G, \mathbb{F})$ where $G:=\langle\bar{u}, \bar{x}, \bar{a} \mid \Sigma\rangle$ be a tower over $\mathbb{F}$. Let $\phi(\bar{x}, \bar{a})$ be a first order formula over $\mathbb{F}$. Suppose there exists a test sequence, $\left(h_{n}\right)_{n<\omega}: G \rightarrow \mathbb{F}$ for $\mathcal{T}(G, \mathbb{F})$, such that $\mathbb{F} \models \phi\left(h_{n}(\bar{x}), \bar{a}\right)$. 
Then there exist:

- a closure, $\mathcal{R}:=\operatorname{cl}(\mathcal{T}(G, \mathbb{F}))$, of $\mathcal{T}(G, \mathbb{F})$;

- finitely many closures, $\mathcal{R}_{1}:=\operatorname{cl}_{1}(\mathcal{R}), \ldots, \mathcal{R}_{k}:=\operatorname{cl}_{k}(\mathcal{R})$, of $\mathcal{R}$;

- for each $i \leq k$, finitely many closures, $c_{1}\left(\mathcal{R}_{i}\right), \ldots, c_{m_{i}}\left(\mathcal{R}_{i}\right)$,

so that a subsequence of $\left(h_{n}\right)_{n<\omega}$ extends to a test sequence of $\mathcal{R}$ and either:

- it cannot be extended to a test sequence for any of the closures $\mathcal{R}_{i}$ or

- for each $i \leq k$ that can be extended to a test sequence for $\mathcal{R}_{i}$, there exists $1 \leq j \leq m_{i}$ so that it extends to a test sequence for $c_{j}\left(\mathcal{R}_{i}\right)$ of $\mathcal{R}_{i}$.

Finally, for any test sequence, $\left(h_{n}^{\prime}\right)_{n<\omega}: G^{c l} \rightarrow \mathbb{F}$, for $\mathcal{R}$ for which one of the above conditions holds, there exists $n_{0}<\omega$ such that $\mathbb{F} \models \phi\left(h_{n}^{\prime}(\bar{x}), \bar{a}\right)$ for all $n>n_{0}$.

The above fact has some strong consequences regarding definability. We record some corollaries that resolve some long-standing questions.

A first order formula $\phi(x)$ is generic in a group $G$ if finitely many translates of the solution set of the formula in $G$ cover $G$.

Corollary 6.27. A first order formula $\phi(x)$ over $\mathbb{F}$ is generic if and only if it contains a test sequence for the tower $\mathcal{T}(\langle x\rangle * \mathbb{F}, \mathbb{F})$.

We note that the following Corollary has been proved by C. Perin using different methods.

Corollary 6.28. Let $c \in \mathbb{F} \backslash\{1\}$. Then the induced structure on $\left(C_{F}(c), \cdot\right)$ (seen as a subgroup of $(\mathbb{F}, \cdot))$ is the structure of a pure group; i.e., every definable set in the induced structure can be defined by multiplication alone.

Proof. By [20] and [21] it is enough to prove that every infinite definable subset of $C_{F}(c)$ is generic in $C_{F}(c)$. Let $X$ be an infinite definable subset of $C_{\mathbb{F}}(c)=\langle\gamma\rangle$. Then we can extract from $X$ a test sequence $\left(b_{n}, \bar{a}\right)_{n<\omega}$ for the tower $\mathcal{T}\left(\langle x, z \mid[x, z]\rangle *_{z=\gamma} \mathbb{F}, \mathbb{F}\right)$, obtained by gluing an infinite cyclic group along the centralizer of $c$ in $\mathbb{F}$.

According to the definition of a test sequence and Theorem 6.26 there exist test sequences $\left(\gamma^{k n+l}\right)_{n<\omega}$ and $\left(\gamma^{-k n+l}\right)_{n<\omega}$ for some natural numbers $k>0$ and $l \geq 0$ such that all but finitely many elements of them belong to the definable set $X$. Thus, $X$ is generic in $C_{\mathbb{F}}(c)$.

6.4. Implicit function theorems. We fix a nonabelian free group $\mathbb{F}:=\mathbb{F}(\bar{a})$. In this section we record some generalizations of Merzlyakov's theorem. Merzlyakov's original theorem stated:

Theorem 6.29 (Merzlyakov). Let $\Sigma(\bar{x}, \bar{y}, \bar{a}) \subset_{\text {finite }}\langle\bar{x}, \bar{y}\rangle * \mathbb{F}$ be a finite set of words. Suppose $\mathbb{F} \models \forall \bar{x} \exists \bar{y}(\Sigma(\bar{x}, \bar{y}, \bar{a})=1)$. Then there exists a "formal solution" $\bar{w}(\bar{x}, \bar{a}) \subset\langle\bar{x}\rangle * \mathbb{F}$ such that $\Sigma(\bar{x}, \bar{w}(\bar{x}, \bar{a}), \bar{a})$ is trivial in $\langle\bar{x}\rangle * \mathbb{F}$.

The above theorem is the conceptual basis of the positive solution to Tarski's problem. The first step towards the solution consists of generalizations of Merzlyakov's theorem to varieties corresponding to limit groups that have the structure of a tower (see [16, Corollary 2.5] and [5, Theorem 12]).

Theorem 6.30. Let $\Sigma(\bar{x}, \bar{y}, \bar{a}) \subset_{\text {finite }}\langle\bar{x}, \bar{y}\rangle * \mathbb{F}$ be a finite set of words. Let $\mathcal{T}(G, \mathbb{F})$ with $G:=\langle\bar{x}, \bar{a} \mid T(\bar{x}, \bar{a})\rangle$ be a tower over $\mathbb{F}$. Let $\mathbb{F} \models \forall \bar{x}(T(\bar{x}, \bar{a})=$ $1 \rightarrow \exists \bar{y}(\Sigma(\bar{x}, \bar{y}, \bar{a})=1))$. 
Then there exist finitely many closures, $\operatorname{cl}_{1}(\mathcal{T}(G, \mathbb{F})), \ldots, c l_{k}(\mathcal{T}(G, \mathbb{F}))$ with $G^{c l_{i}}$ $:=\left\langle\bar{u}_{i}, \bar{x}, \bar{a}\right\rangle$, and for each $i \leq k$ a "formal solution" $\bar{w}_{i}\left(\bar{u}_{i}, \bar{x}, \bar{a}\right)$ such that $\Sigma\left(\bar{x}, \bar{w}_{i}\left(\bar{u}_{i}, \bar{x}, \bar{a}\right), \bar{a}\right)$ is trivial in $G^{c l_{i}}$.

Moreover, for any morphism $h: G \rightarrow \mathbb{F}$ that factors through $\mathcal{T}(G, \mathbb{F})$, there exists some $i \leq k$ such that $h$ extends to a morphism from $G^{c_{i}}$ to $\mathbb{F}$ that factors through $c l_{i}(\mathcal{T}(G, \mathbb{F}))$.

One can generalize Merzlyakov's theorem after strengthening the assumptions in the following way.

Theorem 6.31. Let $\mathbb{F} \models \forall \bar{x} \exists<\infty \bar{y} \phi(\bar{x}, \bar{y}, \bar{a})$ and assume there exist a test sequence $\left(h_{n}\right)_{n<\omega}: G \rightarrow \mathbb{F}$ with respect to the hyperbolic tower $\mathcal{T}(G, \mathbb{F}), G:=\langle u, x, a|$ $T(u, x, a)\rangle$ (for some ordering $(\mathcal{T}(G, \mathbb{F}),<)$ ) and a sequence of tuples $\left(\bar{c}_{n}\right)_{n<\omega}$ in $\mathbb{F}$ such that $\mathbb{F}=\phi\left(h_{n}(\bar{x}), \bar{c}_{n}, \bar{a}\right)$. Then there exists a tuple of words $\bar{w}(\bar{x}, \bar{a}) \subset G$ such that for any test sequence $\left(h_{n}^{\prime}\right)_{n<\omega}: G \rightarrow \mathbb{F}$ for the tower $\mathcal{T}(G, \mathbb{F})$, there exists $n_{0}$ (that depends on the test sequence) with $\mathbb{F} \models \phi\left(h_{n}^{\prime}(\bar{x}), h_{n}^{\prime}(\bar{w}(\bar{x}, \bar{a})), \bar{a}\right)$ for all $n>n_{0}$.

If $h: G \rightarrow \mathbb{F}$ is a morphism and $H$ is a subgroup of $G$, then by $h \uparrow H$ we denote the restriction of $h$ on $H$. We prove:

Theorem 6.32. Let $\mathcal{T}(G, \mathbb{F})$ be a tower over $\mathbb{F}:=\mathbb{F}(\bar{a})$ with $G:=\langle\bar{u}, \bar{y}, \bar{a}\rangle$, and let Sld $:=\langle\bar{v}, \bar{y}, \bar{a} \mid \Sigma(\bar{v}, \bar{y}, \bar{a})\rangle$ be a solid limit group with respect to $H:=\langle\bar{y}, \bar{a}\rangle \leq G$.

Assume that for some ordering of the tower $(\mathcal{T}(G, \mathbb{F}),<)$ there exists a test sequence $\left(h_{n}\right)_{n<\omega}: G \rightarrow \mathbb{F}$ that extends to a sequence $\left(H_{n}\right)_{n<\omega}: G *_{H}$ Sld $\rightarrow \mathbb{F}$ so that for each $n<\omega,\left(H_{n}\lceil S l d)_{n<\omega}\right.$ is a strictly solid morphism.

Then:

- (Existence for a single test sequence) There exist a closure $\operatorname{cl}(\mathcal{T}(G, \mathbb{F})$ ) of $\mathcal{T}(G, \mathbb{F})$ with $G^{c l}:=\langle\bar{w}, \bar{u}, \bar{y}, \bar{a}\rangle$ and a morphism $r:$ Sld $\rightarrow G^{\text {cl }}$ with $r(\bar{y}, \bar{a})=(\bar{y}, \bar{a})$ such that a subsequence of $\left(h_{n}\right)_{n<\omega}\left(\right.$ still denoted $\left.\left(h_{n}\right)_{n<\omega}\right)$ extends to a test sequence $\left(g_{n}\right)_{n<\omega}: G^{c l} \rightarrow \mathbb{F}$ of $\operatorname{cl}(\mathcal{T}(G, \mathbb{F}))$ for the inherited ordering and for which, for each $n, g_{n} \circ r$ is in the same strictly solid family as $H_{n} \uparrow$ Sld.

- (Universal property for all test sequences) There exist finitely many closures $\operatorname{cl}_{1}(\mathcal{T}(G, \mathbb{F})), \ldots, c l_{k}(\mathcal{T}(G, \mathbb{F}))$ with $G^{c l_{i}}:=\left\langle\bar{w}_{i}, \bar{u}, \bar{y}, \bar{a}\right\rangle$, and for each $i \leq k$ there is a morphism $r_{i}: S l d \rightarrow G^{c l_{i}}$ with $r_{i}(\bar{y}, \bar{a})=(\bar{y}, \bar{a})$ such that for every test sequence with respect to $(\mathcal{T}(G, \mathbb{F})<),\left(h_{n}\right)_{n<\omega}: G \rightarrow \mathbb{F}$, which extends to a sequence $\left(H_{n}\right): G *_{H} S l d \rightarrow \mathbb{F}$ so that, for each $n<\omega, H_{n} \uparrow$ Sld is a strictly solid morphism, there exists $i \leq k$ such that a subsequence of $\left(h_{n}\right)_{n<\omega}$ extends to a test sequence $\left(g_{n}\right)_{n<\omega}: G^{c l_{i}} \rightarrow \mathbb{F}$ for the inherited ordering and, for each $n<\omega, g_{n} \circ r_{i}$ is in the same strictly solid family as $H_{n} \uparrow S l d$.

We first record a result of Sela (see [17, Proposition 1.9]) which is essential in proving Theorem 6.32

Proposition 6.33 (Sela). Let Sld be a solid limit group with respect to a finitely generated subgroup $H$. Let $\left(s_{n}\right)_{n<\omega}:$ Sld $\rightarrow \mathbb{F}$ be a converging sequence of strictly solid morphisms. Let $q:$ Sld $\rightarrow Q:=S l d / \operatorname{Ker} s_{n}$ and let $\Delta$ be a GAD of $Q$ relative to $q(H)$. 
Then for each edge group and rigid (nonabelian) vertex group of $J S J_{H}(S l d)$, its image by $q$ can be either

- conjugated into an edge group or rigid (nonabelian) vertex group of $\Delta$ or

- if conjugated into an abelian vertex group, then it is conjugated into the peripheral subgroup of this group.

Proof of Theorem 6.32. The proof follows the arguments in the proof of Theorem 1.18 in [16]. Thus we only point out the parts that differ.

We note that by Lemma 5.9 there exists a system of equations $\Psi=1$ over $\mathbb{F}$ that collects all morphisms $h: S l d \rightarrow \mathbb{F}$ that are degenerate.

We start with a test sequence $\left(h_{n}\right)_{n<\omega}: G \rightarrow \mathbb{F}$ that by the hypothesis we can extend to a sequence of morphisms $\left(H_{n}\right)_{n<\omega}: G *_{H} S l d \rightarrow \mathbb{F}$ such that each morphism restricts to a strictly solid morphism on Sld. For each $n$ we choose the shortest possible morphism (with respect to a fixed basis of $\mathbb{F}$ ) that belongs to the same strictly solid family as $H_{n} \uparrow S l d$. The problem in applying directly the arguments of the proof of Theorem 1.18 in [16] would be that the shortening argument could shorten our morphisms in a way that they do not belong in the same strictly solid family. According to Proposition 6.33 and Lemma 5.11 this is not possible. Thus, the quotient group $G *_{H} S l d / \operatorname{Ker}\left(H_{n}\right)$ under the stable kernel of $\left(H_{n}\right)_{n<\omega}$ would have the structure of a closure of $\mathcal{T}(G, \mathbb{F})$, as we wanted.

The universal property follows exactly as in Theorem 1.18 in [16].

Theorem 6.31 is an easy corollary of our next result.

Theorem 6.34. Let $\mathcal{T}(G, \mathbb{F})$ with $G:=\langle\bar{u}, \bar{y}, \bar{a} \mid T(\bar{u}, \bar{y}, \bar{a})\rangle$ be a tower over $\mathbb{F}$. Let $\{\phi(\bar{x}, \bar{y}, \bar{a})\}$ be a parametric family of first order formulas with respect to $\bar{y}$ such that $\mathbb{F} \models \forall \bar{y} \exists<\infty \bar{x} \phi(\bar{x}, \bar{y}, \bar{a})$.

Suppose there exist a test sequence $\left(h_{n}\right)_{n<\omega}: G \rightarrow \mathbb{F}$ for $\mathcal{T}(G, \mathbb{F})$ and a sequence $\left\{\left(\bar{b}_{n}\right)_{n<\omega}\right\}$ of tuples in $\mathbb{F}$ such that $\mathbb{F} \models \phi\left(\bar{b}_{n}, h_{n}(\bar{y}), \bar{a}\right)$.

Then there exist finitely many closures $\operatorname{cl}_{1}(\mathcal{T}(G, \mathbb{F})), \ldots, c l_{k}(\mathcal{T}(G, \mathbb{F}))$ with $G^{c_{i}}$ $:=\left\langle\bar{v}_{i}, \bar{u}, \bar{y}, \bar{a} \mid \operatorname{cl}_{i}(T)\left(\bar{v}_{i}, \bar{u}, \bar{y}, \bar{a}\right)\right\rangle$ and for each $i \leq k$ a tuple of words $\bar{x}_{i}:=$ $\bar{x}_{i}\left(\bar{v}_{i}, \bar{u}, \bar{y}, \bar{a}\right)$ such that for any test sequence $\left(h_{n}^{\prime}\right)_{n<\omega}: G \rightarrow \mathbb{F}$ for $\mathcal{T}(G, \mathbb{F})$ for which there exists $\left\{\left(\bar{b}_{n}\right)_{n<\omega}\right\}$ with $\mathbb{F} \models \phi\left(\bar{b}_{n}, h_{n}^{\prime}(\bar{y}), \bar{a}\right)$, there exists $i \leq k$, and a subsequence of $\left(h_{n}^{\prime}\right)_{n<\omega}$ extends to a test sequence $\left(g_{n}\right)_{n<\omega}: G^{c_{i}} \rightarrow \mathbb{F}$ for $c_{i}(\mathcal{T}(G, \mathbb{F})$ ) with $\mathbb{F} \mid=\phi\left(g_{n}\left(\bar{x}_{i}\right), g_{n}(\bar{y}), \bar{a}\right)$.

Proof. We consider the solid limit groups on which the graded towers of the envelope of $\{\phi(\bar{x}, \bar{y}, \bar{a})\}$ are based. By Theorem 6.25 the tuple $\bar{x}$ of each graded tower belongs to $\hat{H}_{i}$ of each $J S J\left(S l d_{i}\right)$. By the properties of the graded envelope every test sequence for $\mathcal{T}(G, \mathbb{F})$ that can be extended to a sequence of solutions for $\phi$ has a subsequence that extends to a sequence of morphisms of $G *_{H_{i}} S l d_{i}$, so $h_{n}^{\prime}$ extends to $H_{n}^{\prime}: G *_{H_{i}} S l d_{i}$. Now by Theorem 6.32 there are closures $c l_{1}, \ldots, c l_{n}$ with $x_{i}=r_{i}(x)$ such that $h_{n}^{\prime}$ extends to $g_{n}: G^{c l_{i}} \rightarrow \mathbb{F}$. Since $g_{n} \circ r$ is in the same strictly solid family as $H_{n}^{\prime} \uparrow S l d$, by Theorem 6.25 it does not change the value of $H_{n}^{\prime}\left(x_{i}\right)=b_{n}$; that is, $\mathbb{F} \models \phi\left(g_{n}\left(x_{i}\right), h_{n}^{\prime}(y), a\right)$.

\section{MAIN PROOF}

In this final section we bring everything together in order to prove the main result of this paper: no infinite field is definable in a nonabelian free group. We have split the proof into two parts. Assuming that $X$ is an infinite definable set, in subsection 7.1 we tackle the case where $X$ is coordinated by a finite set of centralizers. In this 
case we have already proved that $X$ is one-based; thus we cannot give it a definable field structure. In subsection 7.3 we prove that in any other case $X$ cannot be given definably the structure of an abelian group.

We have inserted a subsection between subsections 7.1 and 7.3 that is free of certain technical problems in order to make the ideas of our proof more transparent.

7.1. Abelian case. In this subsection we tackle the special case where the diophantine envelope of a definable set $X$ consists essentially of the ground floor $\mathbb{F}$ (the coefficient group) and only abelian floors are glued over centralizers of elements in $\mathbb{F}$, the prototypical case being that $X$ is a product of centralizers. Since centralizers are pure groups (see Corollary 6.28, their theory is one-based; thus $X$ cannot be given definably a field structure. In the general case we can show that $X$ is internal to a product of centralizers, thus still one-based.

Theorem 7.1. Let $X:=\phi(\bar{x}, \bar{a})$ be a first order formula over $\mathbb{F}$ with $|X|=\infty$. Let $\left\{\left(\mathcal{T}\left(G_{i}, \mathbb{F}\right)\right)_{i \leq k}\right\}$, where $G_{i}:=\left\langle\bar{u}_{i}, \bar{x}, \bar{a} \mid \Sigma_{i}\left(\bar{u}_{i}, \bar{x}, \bar{a}\right)\right\rangle$ is a diophantine envelope for $X$. Suppose, for each $i \leq k$, the tuple $\bar{x}$ belongs to the subtower of $\mathcal{T}_{i}$ consisting of the ground floor $\mathbb{F}$ and the abelian flats glued over centralizers of elements in $\mathbb{F}$.

Then $X$ cannot be given definably the structure of a field.

Proof. By Corollary 6.24(i), we have that $X$ is a subset of the diophantine set $D(\bar{x}):=\exists \bar{u}\left(\bigvee_{i=1}^{k} \Sigma_{i}(\bar{u}, \bar{x}, \bar{a})=1\right)$. But then by the hypothesis we have that there are finitely many words $\bar{w}_{i}(\bar{z}, \bar{a})$ so that for each element $\bar{b}$ of $D$ there exist some $i$ and some elements $\bar{c}_{1}, \ldots, \bar{c}_{l}$ from centralizers of elements in $\mathbb{F}$ such that $\bar{b}=$ $w_{i}\left(\bar{c}_{1}, \ldots, \bar{c}_{l}, \bar{a}\right)$.

Thus, $D$ is internal to a set of centralizers (see Definition 2.6), and by Corollary 6.28 these centralizers are one-based sets. Now by Fact 2.4 and Theorem 2.7 no infinite definable subset of $D$ can be given definably a field structure.

7.2. Hyperbolic case. In this subsection we tackle a case in which a diophantine envelope for a definable set $X$ contains a hyperbolic tower. In contrast to the abelian case, this case is not strictly needed, but it will serve as a toy example for the general case.

Theorem 7.2. Let $X:=\phi(\bar{x}, \bar{a})$ be a first order formula over $\mathbb{F}$ with $|X|=\infty$. Let $\left\{\left(\mathcal{T}_{i}\left(G_{i}, \mathbb{F}\right)\right)_{i \leq k}\right\}$, where $G_{i}:=\left\langle\bar{u}, \bar{x}, \bar{a} \mid \Sigma_{i}\right\rangle$, be a diophantine envelope for $X$. Suppose, for some $i \leq k$, that $\mathcal{T}_{i}$ is hyperbolic.

Then $X$ cannot be given definably an abelian group structure.

Proof. Suppose for the sake of contradiction that $(\phi(\bar{x}, \bar{a}), \oplus(\bar{x}, \bar{y}, \bar{z}, \bar{a}))$ is an abelian group. Let $\mathcal{T}(G, \mathbb{F})$, where $G:=\langle\bar{u}, \bar{x}, \bar{a} \mid \Sigma(\bar{u}, \bar{x}, \bar{a})\rangle$ be the hyperbolic tower contained in a diophantine envelope of $\phi(\bar{x}, \bar{a})$.

We consider the twin tower $\mathcal{T} \# \mathcal{T}(G, \mathbb{F})$, its corresponding group $G *_{\mathbb{F}} G^{\prime}:=$ $\left\langle\bar{u}, \bar{x}, \bar{u}^{\prime}, \bar{x}^{\prime}, \bar{a} \mid \Sigma(\bar{u}, \bar{x}, \bar{a}), \Sigma\left(\bar{u}^{\prime}, \bar{x}^{\prime}, \bar{a}\right)\right\rangle$, and a test sequence $\left(h_{n}\right)_{n<\omega}: G *_{\mathbb{F}} G^{\prime} \rightarrow \mathbb{F}$ for $\mathcal{T} \# \mathcal{T}$ with respect to the twin tower and its natural order.

We note that by the properties of test sequences the restrictions $\left(h_{n}\right)_{n<\omega} \uparrow G$, $\left(h_{n}\right)_{n<\omega} \uparrow G^{\prime}$ are both test sequences for $\mathcal{T}(G, \mathbb{F})$ (for the natural order). Thus by Theorem 6.26 the sequence of couples $\left(h_{n}(\bar{x}), h_{n}\left(\bar{x}^{\prime}\right)\right)$ belongs to $X \times X$. Therefore, there exists a sequence of tuples $\left(\bar{c}_{n}\right)_{n<\omega}$ of $\mathbb{F}$ such that $\mathbb{F} \models \oplus\left(h_{n}(\bar{x}), h_{n}\left(\bar{x}^{\prime}\right), \bar{c}_{n}\right)$. We can now use Theorem 6.31 in order to obtain a formal solution $\bar{w}\left(\bar{u}, \bar{x}, \bar{u}^{\prime}, \bar{x}^{\prime}, \bar{a}\right)$, i.e., a tuple of words in $G *_{\mathbb{F}} G^{\prime}$, such that

$$
\mathbb{F} \models \oplus\left(h_{n}(\bar{x}), h_{n}\left(\bar{x}^{\prime}\right), h_{n}\left(\bar{w}\left(\bar{u}, \bar{x}, \bar{u}^{\prime}, \bar{x}^{\prime}, \bar{a}\right)\right), \bar{a}\right) .
$$


We claim that there is an element in the tuple $\bar{w}\left(\bar{u}, \bar{x}, \bar{u}^{\prime}, \bar{x}^{\prime}, \bar{a}\right)$ that does not belong to $\mathbb{F}$. If, for a contradiction, $\bar{w}\left(\bar{u}, \bar{x}, \bar{u}^{\prime}, \bar{x}^{\prime}, \bar{a}\right)=\bar{b}$ for some tuple $\bar{b}$ of $\mathbb{F}$, then we can replace $\left(h_{n}\right) \uparrow G^{\prime}$ with a proper subsequence. This will still be a test sequence for $\mathcal{T} \# \mathcal{T}$; call it $\left(g_{n}\right)_{n<\omega}$. By Theorem 6.26 we get $\mathbb{F} \models \oplus\left(g_{n}(\bar{x}), g_{n}\left(\bar{x}^{\prime}\right), \bar{b}, \bar{a}\right)$, but $g_{n}(\bar{x})=h_{n}(\bar{x})$, while for $n$ large enough $g_{n}\left(\bar{x}^{\prime}\right) \neq h_{n}\left(\bar{x}^{\prime}\right)$. Thus $h_{n}(\bar{x}) \oplus h_{n}\left(\bar{x}^{\prime}\right) \neq$ $h_{n}(\bar{x}) \oplus g_{n}\left(\bar{x}^{\prime}\right)$, a contradiction since both should be equal to $\bar{b}$.

We next consider exchanging the role of $\left(h_{n}\right)_{n<\omega} \uparrow G$ with $\left(h_{n}\right)_{n<\omega} \uparrow G^{\prime}$ since $G^{\prime}$ is a copy of $G$. This is again a sequence of morphisms from $G *_{\mathbb{F}} G^{\prime}$ to $\mathbb{F}$, which we call $\left(f_{n}\right)_{n<\omega}$. The sequence $\left(f_{n}\right)_{n<\omega}$ is a test sequence for $\mathcal{T} \# \mathcal{T}$ but for a different order: namely, the floors that consist of the group $G^{\prime}$ come before the floors that consist of the group $G$. Thus, we can still apply Theorem 6.26 so that $\mathbb{F}=\oplus\left(f_{n}(\bar{x}), f_{n}\left(\bar{x}^{\prime}\right), f_{n}\left(\bar{w}\left(\bar{u}, \bar{x}, \bar{u}^{\prime}, \bar{x}^{\prime}, \bar{a}\right)\right), \bar{a}\right)$. By the definition of $\left(f_{n}\right)_{n<\omega}$ it follows that $h_{n}(\bar{x})=f_{n}\left(\bar{x}^{\prime}\right)$ and $h_{n}\left(\bar{x}^{\prime}\right)=f_{n}(\bar{x})$. In particular, since the group operation $\oplus$ is abelian we observe that

$$
h_{n}\left(\bar{w}\left(\bar{u}, \bar{x}, \bar{u}^{\prime}, \bar{x}^{\prime}, \bar{a}\right)\right)=f_{n}\left(\bar{w}\left(\bar{u}, \bar{x}, \bar{u}^{\prime}, \bar{x}^{\prime}, \bar{a}\right)\right)=h_{n}\left(\bar{w}\left(\bar{u}^{\prime}, \bar{x}^{\prime}, \bar{u}, \bar{x}, \bar{a}\right)\right) .
$$

In particular the equation $\left.\left.\bar{w}\left(\bar{u}, \bar{x}, \bar{u}^{\prime}, \bar{x}^{\prime}, \bar{a}\right)\right)=\bar{w}\left(\bar{u}^{\prime}, \bar{x}^{\prime}, \bar{u}, \bar{x}, \bar{a}\right)\right)$ holds for a test sequence with respect to $\mathcal{T} \# \mathcal{T}$. But a test sequence witnesses that $G *_{\mathbb{F}} G^{\prime}$ is a limit group. Thus the above equation is a formal relation in the corresponding group $G *_{\mathbb{F}} G^{\prime}$. Now since $\left.\bar{w}\left(\bar{u}, \bar{x}, \bar{u}^{\prime}, \bar{x}^{\prime}, \bar{a}\right)\right)$ does not live in $\mathbb{F}$, some element in $\left.\bar{w}\left(\bar{u}, \bar{x}, \bar{u}^{\prime}, \bar{x}^{\prime}, \bar{a}\right)\right)$ and the corresponding element in $\left.\bar{w}\left(\bar{u}^{\prime}, \bar{x}^{\prime}, \bar{u}, \bar{x}, \bar{a}\right)\right)$ have different normal forms with respect to the amalgamated free product $G *_{\mathbb{F}} G^{\prime}$, a contradiction.

\subsection{General case.}

Theorem 7.3. Let $X:=\phi(\bar{x}, \bar{a})$ be a first order formula over $\mathbb{F}$ with $|X|=\infty$. Then $X$ cannot be given definably a field structure.

Proof. Let $\left\{\left(\mathcal{T}\left(G_{i}, \mathbb{F}\right)\right)_{i \leq k}\right\}$ be a diophantine envelope of $\phi(\bar{x}, \bar{a})$. By Theorem 7.1 we may assume that there exists a tower $\mathcal{T}(G, \mathbb{F})($ where $G:=\langle\bar{u}, \bar{x}, \bar{a}| \Sigma(\bar{u}, \bar{x}, \bar{a}\rangle)$ in the diophantine envelope such that either the first floor is not an abelian floor or the tuple of elements $\bar{x}$ does not live in the group that corresponds to the first floor. We next prove that in either case $X$ cannot be given definably an abelian group structure. Assume for a contradiction that $\left(X, \oplus\left(\bar{x}, \bar{x}^{\prime}, \bar{z}\right)\right)$ for some first order formula $\oplus\left(\bar{x}, \bar{x}^{\prime}, \bar{z}\right)$ is an abelian group.

We first consider the case where the first floor, $\mathcal{G}\left(G^{1}, \mathbb{F}\right)$, of the tower $\mathcal{T}(G, \mathbb{F})$ is not abelian. Let $G^{\prime}:=\left\langle\bar{u}^{\prime}, \bar{x}^{\prime}, \bar{a} \mid \Sigma\left(\bar{u}^{\prime}, \bar{x}^{\prime}, \bar{a}\right)\right\rangle$ be a copy of $G$. We consider the twin tower $\mathcal{T} \# \mathcal{T}\left(G *_{\mathbb{F}} G^{\prime}, \mathbb{F}\right)$, where $G *_{\mathbb{F}} G^{\prime}:=\left\langle\bar{u}, \bar{x}, \bar{u}^{\prime}, \bar{x}^{\prime}, \bar{a} \mid \Sigma(\bar{u}, \bar{x}, \bar{a}), \Sigma\left(\bar{u}^{\prime}, \bar{x}^{\prime}, \bar{a}\right)\right\rangle$ and the finite set of closures $c l_{1}\left(\mathcal{T} \# \mathcal{T}\left(G *_{\mathbb{F}} G^{\prime}, \mathbb{F}\right)\right), \ldots, c l_{k}\left(\mathcal{T} \# \mathcal{T}\left(G *_{\mathbb{F}} G^{\prime}, \mathbb{F}\right)\right)$ of the twin tower provided by applying Theorem 6.34 to the twin tower $\mathcal{T} \# \mathcal{T}\left(G *_{\mathbb{F}} G^{\prime}, \mathbb{F}\right)$ and the first order formula $\oplus\left(\bar{x}, \bar{x}^{\prime}, \bar{z}\right)$ can be seen as a parametric family with respect to $\bar{x}, \bar{x}^{\prime}$. According to Lemma 4.31, we may assume those closures are symmetric closures.

We now choose a test sequence for the twin tower $\mathcal{T} \# \mathcal{T}\left(G *_{\mathbb{F}} G^{\prime}, \mathbb{F}\right)$ such that $\left(h_{n}(\bar{x}), h_{n}\left(\bar{x}^{\prime}\right)\right)$ belongs to $X \times X$. Moreover, if $K_{i}$ is the coefficient matrix corresponding to the closure embedding $f: E \oplus \mathbb{Z}^{m} \rightarrow E \oplus \mathbb{A}^{m}$ in the $i$-th closure, then the vectors of integers corresponding to $h \uparrow \mathbb{Z}^{m}$ and $h \uparrow \hat{\mathbb{Z}^{m}}$ (see Remark 4.25) belong to the same coset for each $1 \leq i \leq k$. By Theorem 6.34, such a test sequence must extend to a test sequence $\left(h_{n}^{\prime}\right)_{n<\omega}$ of one of the closures, say 
$c l_{1}\left(\mathcal{T} \# \mathcal{T}\left(G *_{\mathbb{F}} G^{\prime}, \mathbb{F}\right)\right)$, giving a formal solution for $z$ in $c l_{1}\left(\mathcal{T} \# \mathcal{T}\left(G *_{\mathbb{F}} G^{\prime}, \mathbb{F}\right)\right)$, and by reversing the role of $\left(h_{n}\right)_{n<\omega} \uparrow G$ with $\left(h_{n}\right)_{n<\omega}\left\lceil G^{\prime}\right.$ we obtain a test sequence that extends to the same closure. We can now apply the same argument as in the case of the hyperbolic tower.

Finally, we are left with the case where the first floor $\mathcal{G}\left(G^{1}, \mathbb{F}\right)$ is abelian. In this case the twin tower has a slightly different form (see Proposition 4.22); as a group it is the amalgamated free product of $G_{D b}$ with ${ }_{f} G_{D b}$ over the double $G_{D b}^{1}$ of $G^{1}$ with respect to the floor $\mathcal{G}\left(G^{1}, \mathbb{F}\right)$. In the first step of our previous argument we applied Theorem 6.34 in order to obtain finitely many closures and formal solutions in these closures so that their images under a test sequence give us the product operation. In order to conclude as in the previous case it is enough to show that these formal solutions do not live in the closure of $G_{D b}^{1}$ for each closure of the twin tower. Indeed, the values of such a formal solution would depend only on the values a test sequence $\left(h_{n}\right)_{n<\omega}: G_{D b} *_{G_{D b}^{1}}\left({ }_{f} G_{D b} \rightarrow \mathbb{F}\right.$ of the twin tower gives to the elements of $G_{D b}^{1}$. But as we have assumed that $\bar{x}$ does not live in $G^{1}$, we can refine the test sequence $\left(h_{n}\right)_{n<\omega}$ as follows: $\left(h_{n}^{\prime}\right)_{n<\omega} \uparrow G_{D b}=\left(h_{n}\right)_{n<\omega} \uparrow G_{D b}$, and $\left(h_{n}^{\prime}\right)_{n<\omega} \uparrow{ }_{f} G_{D b}$ is a sequence that eventually gives to $\bar{x}^{\prime}$ different values from $\left(h_{n}\right)_{n<\omega}$. Moreover, we choose $h_{n}^{\prime}$ so that it has the same properties listed in the previous case, assuring that if it extends to the $i$-th closure, then also the test sequence obtained by reversing the order extends to the same closure. Now, as in the hyperbolic case, since the product of $h_{n}(\bar{x}), h_{n}\left(\bar{x}^{\prime}\right)$ depends only on the values $h_{n}$ gives to the elements of the group $G_{D b}^{1}$, there is a contradiction.

\section{REFERENCES}

[1] Mladen Bestvina, Degenerations of the hyperbolic space, Duke Math. J. 56 (1988), no. 1, 143-161, DOI 10.1215/S0012-7094-88-05607-4. MR.932860

[2] Mladen Bestvina and Mark Feighn, Notes on Sela's work: limit groups and Makanin-Razborov diagrams, Geometric and cohomological methods in group theory, London Math. Soc. Lecture Note Ser., vol. 358, Cambridge Univ. Press, Cambridge, 2009, pp. 1-29. MR2605174

[3] Vincent Guirardel, Actions of finitely generated groups on $\mathbb{R}$-trees (English, with English and French summaries), Ann. Inst. Fourier (Grenoble) 58 (2008), no. 1, 159-211. MR2401220

[4] O. Kharlampovich and A. Myasnikov, Irreducible affine varieties over a free group. I. Irreducibility of quadratic equations and Nullstellensatz, J. Algebra 200 (1998), no. 2, 472-516, DOI 10.1006/jabr.1997.7183. MR.1610660

[5] Olga Kharlampovich and Alexei Myasnikov, Implicit function theorem over free groups, J. Algebra 290 (2005), no. 1, 1-203, DOI 10.1016/j.jalgebra.2005.04.001. MR2154989

[6] Olga Kharlampovich and Alexei Myasnikov, Elementary theory of free non-abelian groups, J. Algebra 302 (2006), no. 2, 451-552, DOI 10.1016/j.jalgebra.2006.03.033. MR 2293770

[7] Olga Kharlampovich and Alexei Myasnikov, Definable sets in a hyperbolic group, Internat. J. Algebra Comput. 23 (2013), no. 1, 91-110, DOI 10.1142/S021819671350001X. MR3040804

[8] Frédéric Paulin, Topologie de Gromov équivariante, structures hyperboliques et arbres réels (French), Invent. Math. 94 (1988), no. 1, 53-80, DOI 10.1007/BF01394344. MR958589

[9] Chloé Perin, Anand Pillay, Rizos Sklinos, and Katrin Tent, On groups and fields interpretable in torsion-free hyperbolic groups, Münster J. Math. 7 (2014), no. 2, 609-621. MR3426232

[10] Chloé Perin and Rizos Sklinos, Forking and JSJ decomposition in the free group, J. Eur. Math. Soc. (JEMS) 18 (2016), no. 9, 1983-2017, DOI 10.4171/JEMS/636. MR3531668

[11] Anand Pillay, Geometric stability theory, Oxford Logic Guides, vol. 32, The Clarendon Press, Oxford University Press, New York, 1996. MR.1429864

[12] Anand Pillay, A note on CM-triviality and the geometry of forking, J. Symbolic Logic 65 (2000), no. 1, 474-480, DOI 10.2307/2586549. MR1782132

[13] Anand Pillay, Forking in the free group, J. Inst. Math. Jussieu 7 (2008), no. 2, 375-389, DOI 10.1017/S1474748008000066. MR2400726 
[14] Zlil Sela, Diophantine geometry over groups IX: Envelopes and imaginaries, preprint, available at http://www.ma.huji.ac.il/ zlil/.

[15] Zlil Sela, Diophantine geometry over groups. I. Makanin-Razborov diagrams, Publ. Math. Inst. Hautes Études Sci. 93 (2001), 31-105, DOI 10.1007/s10240-001-8188-y. MR.1863735

[16] Z. Sela, Diophantine geometry over groups. II. Completions, closures and formal solutions, Israel J. Math. 134 (2003), 173-254, DOI 10.1007/BF02787407. MR1972179

[17] Z. Sela, Diophantine geometry over groups. III. Rigid and solid solutions, Israel J. Math. 147 (2005), 1-73, DOI 10.1007/BF02785359. MR2166355

[18] Z. Sela, Diophantine geometry over groups. VII. The elementary theory of a hyperbolic group, Proc. Lond. Math. Soc. (3) 99 (2009), no. 1, 217-273, DOI 10.1112/plms/pdn052. MR2520356

[19] Jean-Pierre Serre, Arbres, amalgames, $\mathrm{SL}_{2}$ (French), Avec un sommaire anglais; Rédigé avec la collaboration de Hyman Bass, Astérisque, No. 46, Société Mathématique de France, Paris, 1977. MR0476875

[20] Rizos Sklinos, Some model theory of the free group, Ph.D. thesis, University of Leeds, UK, 2011.

[21] Rizos Sklinos, The free group does not have the finite cover property, Israel J. Math. 227 (2018), no. 2, 563-595, DOI 10.1007/s11856-018-1748-3. MR.3846335

[22] Frank O. Wagner, Some remarks on one-basedness, J. Symbolic Logic 69 (2004), no. 1, 34-38, DOI 10.2178/jsl/1080938823. MR2039343

[23] B. I. Zil'ber, The structure of models of uncountably categorical theories, Proceedings of the International Congress of Mathematicians, Vol. 1, 2 (Warsaw, 1983), PWN, Warsaw, 1984, pp. 359-368. MR804692

Department of Mathematics, Technion - Israel Institute of Technology, Haifa, 3200003, ISRAEL

Department of Mathematics, Schaefer School of Engineering \& Science, Stevens Institute of Technology, Hoboken, New Jersey 07030 